\title{
Founding events and the maintenance of genetic diversity in reintroduced populations
}

Kimberly Anne Miller

\begin{abstract}
A thesis submitted to
Victoria University of Wellington

in fulfilment of the requirements for the degree of

Doctor of Philosophy in Ecology and Biodiversity
\end{abstract}

Victoria University of Wellington

Te Whare Wānanga o te Üpoko o te Ika a Māui

2009 


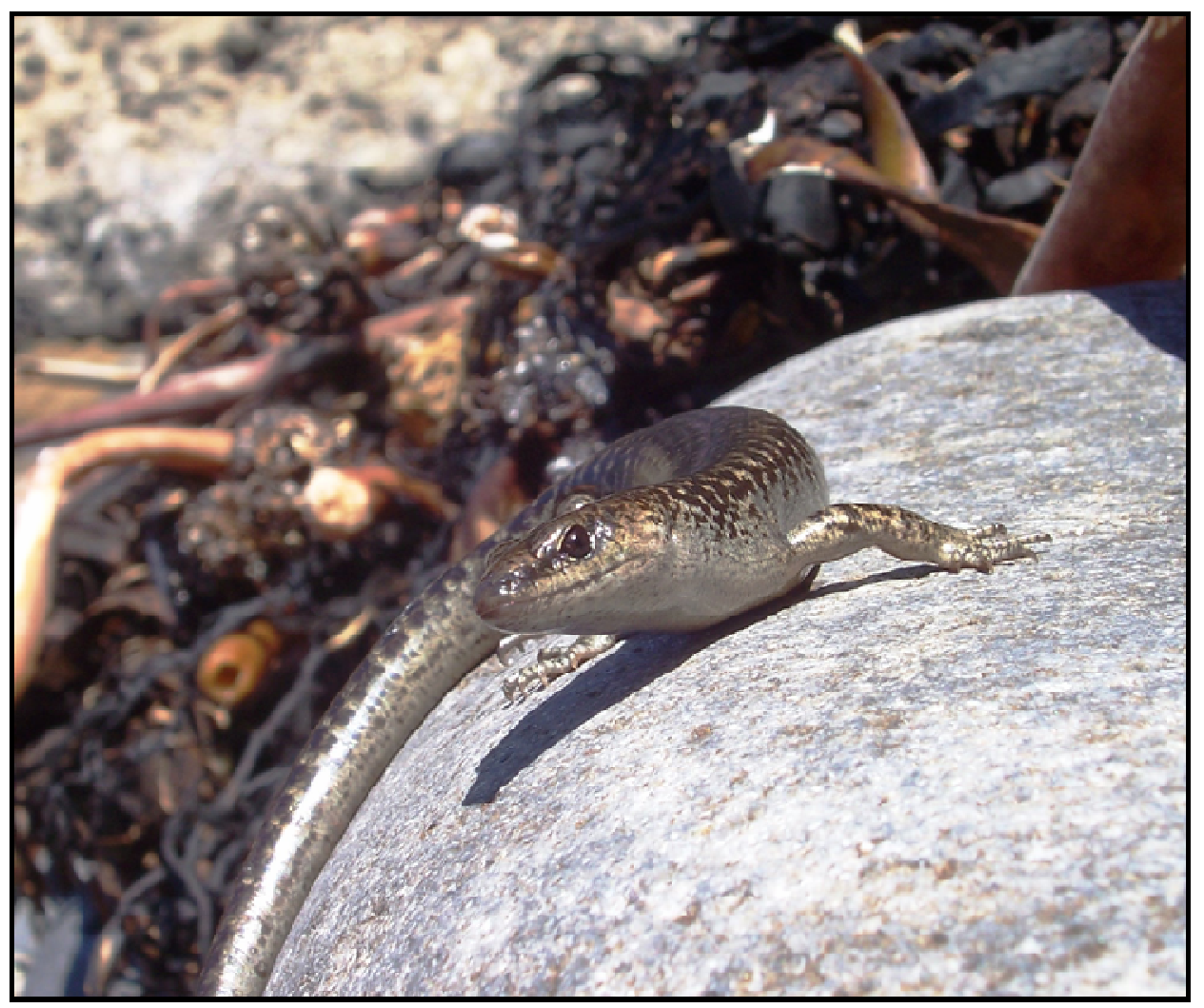

The race is not always to the swift, nor the battle to the strong, but time and chance happens to us all.

Ecclesiastes 9:11 


\section{Abstract}

As habitat loss, introduced predators, and disease epidemics threaten species worldwide, translocation provides one of the most powerful tools for species conservation. However, reintroduced populations of threatened species are often founded by a small number of individuals (typically 30 in New Zealand) and generally have low success rates. The loss of genetic diversity combined with inbreeding depression in a small reintroduced population could reduce the probability of establishment and persistence. Effective management of genetic diversity is therefore central to the success of reintroduced populations in both the short- and long-term. Using population modelling and empirical data from source and reintroduced populations of skinks and tuatara, I examined factors that influence inbreeding dynamics and the long-term maintenance of genetic diversity in translocated populations. The translocation of gravid females aided in increasing the effective population size after reintroduction. Models showed that supplementation of reintroduced populations reduced the loss of heterozygosity over 10 generations in species with low reproductive output, but not for species with higher output. Harvesting from a reintroduced population for a second-order translocation accelerated the loss of heterozygosity in species with low intrinsic rates of population growth. Male reproductive skew also accelerated the loss of genetic diversity over 10 generations, but the effect was only significant when the population size was small. Further, when populations at opposite ends of a species' historic range are disproportionately vulnerable to extinction and background inbreeding is high, genetic differentiation among populations may be an artefact of an historic genetic gradient coupled with rapid genetic drift. In these situations, marked genetic differences should not preclude hybridising populations to mitigate the risks of inbreeding after reintroduction. These results improve translocation planning for many species by offering guidelines for maximising genetic diversity in founder groups and managing populations to improve the long-term maintenance of diversity. For example, founder groups should be larger than 30 for reintroductions of species with low reproductive output, high mortality rates after release, highly polygynous mating systems, and high levels of background inbreeding. This study also provides a basis for the development of more complex models of losses of genetic diversity after translocation and how genetic drift may affect the long-term persistence of these valuable populations. 


\section{Acknowledgements}

My $\mathrm{PhD}$ has been a fantastic ride, supported by many incredible people and organisations. It would not have been possible without Nicky Nelson and Dave Towns, whose unwavering enthusiasm and support of this project kept me going. Thank you. I am also extremely grateful to Charlie Daugherty for providing me with such an amazing opportunity from the start. Thanks also to Fred Allendorf and Pete Ritchie for a number of valuable discussions and advice that greatly improved my papers and thesis.

Kelly Hare and Jen Moore have been cherished supports through it all - whether dazed entertainment in the field, glasses of wine, advice on chapters, or just a good climbing session, you have been invaluable. Charlie's Angels - Dave Chapple, Jo Hoare, Sue Keall, Libby Liggins, Hilary Miller, Gail Porter, Kristina Ramstad, and Jeanine Refsnider - have been great friends, excellent coffee companions, and a wonderful source of advice.

A number of Department of Conservation staff were incredibly supportive of this project. They were always quick with permits and went above and beyond to provide assistance and support whenever and wherever I needed it. Thanks to Lynn Adams, Rob Chappell, Pete Gaze, Jo Greenman, Nicky Marriott, Jason Roxborough, and Matt Sidaway. Dennis Keall graciously allowed me to sample animals in his captive colony on many occasions. His efforts in captive breeding and support of research are an excellent example of the role that private citizens play in conservation.

Dave Towns, Chris Green, Kelly Hare, Shay O'Neill, Monica Gruber, and Sue Keall were invaluable in the field, providing transport, advice, and incredible support. I also had the great pleasure of working with many other enthusiastic and immensely enjoyable people in the field: Ian Atkinson, Isle Boerman, Derek Brown, Daryl Coleman, Susie Cooper, Maggie Drews, Jo Hoare, Zac Holter, Heidy Kikillus, Bob Literman, Richard Parrish, Hannah Rainforth, Jeanine Refsnider, Richard Romijn, Paolo Segre, Lennart Suselbeek, Kiri Wallace, Richard Walle, and Adrienne Wiley.

This project was funded by the New Zealand Department of Conservation, Victoria University of Wellington, the Allan Wilson Centre for Molecular Ecology and Evolution, the Society for Research on Amphibians and Reptiles in New Zealand (SRARNZ), the Centre 
for Biodiversity and Restoration Ecology (CBRE), the Zoological Society of San Diego, and the Foundation for Research, Science \& Technology. I was personally funded by a Victoria University of Wellington PhD scholarship and an Education New Zealand Postgraduate Study Abroad Award. This work was supported by Karori Wildlife Sanctuary and Ngāti Hei, Ngāti Koata, Te Ātiawa, and Wellington Tenths Trusts. Permission was obtained from the Victoria University of Wellington Animal Ethics Committee (2006R12) and the Department of Conservation (permits: WK/19129/RES, WE/240/RES, WK/18528/RES, NM/18922/CAP).

I need to thank my parents, siblings, and Allie, Emily, and Megan for their constant understanding and encouragement. Lastly, I am incredibly grateful to all those who have supported me, whether they knew it or not. Sometimes a good laugh is all you need. 


\section{Table of Contents}

$\begin{array}{lc}\text { Abstract } & \text { iii } \\ \text { Acknowledgements } & \text { iv } \\ \text { Table of Contents } & \text { vi }\end{array}$

CHAPTER ONE. Founding events, inbreeding, and their influence on translocated populations: An overview

$\begin{array}{ll}1.1 \text { Introduction } & 1\end{array}$

1.2 The importance of genetic diversity 1

1.3 Translocations 3

1.4 Genetic diversity in translocated populations 5

$\begin{array}{ll}1.5 \text { Thesis structure } & 6\end{array}$

$\begin{array}{ll}\text { 1.6 Statement of authorship } & 9\end{array}$

CHAPTER TWO. Assessing genetic diversity for conservation management: a case study of a threatened reptile

$\begin{array}{ll}2.1 \text { Abstract } & 10\end{array}$

$\begin{array}{ll}2.2 \text { Introduction } & 10\end{array}$

$\begin{array}{ll}2.3 \text { Methods } & 14\end{array}$

2.3.1 Sample collection and DNA extraction 14

$\begin{array}{ll}\text { 2.3.2 Mitochondrial DNA sequencing } & 14\end{array}$

$\begin{array}{ll}\text { 2.3.3 Microsatellite genotyping } & 15\end{array}$

$\begin{array}{ll}\text { 2.3.4 Data analysis } & 15\end{array}$

$\begin{array}{ll}2.4 \text { Results } & 16\end{array}$

$\begin{array}{ll}\text { 2.4.1 MtDNA variation } & 16\end{array}$

$\begin{array}{ll}\text { 2.4.2 Microsatellite variation within populations } & 17\end{array}$

$\begin{array}{ll}2.5 \text { Discussion } & 20\end{array}$

$\begin{array}{ll}\text { 2.5.1 Management implications } & 23\end{array}$

CHAPTER THREE. Genetic structure and individual fitness following translocation of a small lizard

$\begin{array}{ll}3.1 \text { Abstract } & 24\end{array}$

$\begin{array}{ll}3.2 \text { Introduction } & 24\end{array}$

3.2.1 Translocation of the egg-laying skink 26 
$\begin{array}{lr}\text { 3.3 Methods } & 28\end{array}$

3.3.1 Animal collection and microsatellite genotyping 28

$\begin{array}{ll}\text { 3.3.2 Fitness measures } & 28\end{array}$

3.3.3 Data analysis $\quad 29$

3.4 Results 33

3.4.1 Genetic bottleneck effects 33

$\begin{array}{ll}\text { 3.4.2 Estimates of effective population size } & 35\end{array}$

3.4.3 Inbreeding and heterozygosity-fitness correlations 37

$\begin{array}{ll}3.5 \text { Discussion } & 39\end{array}$

3.5.1 Genetic bottleneck effects $\quad 40$

3.5.2 Maximising $\mathrm{N}_{\mathrm{e}}$ during translocation $\quad 41$

$\begin{array}{ll}3.5 .3 \text { Fitness consequences } & 42\end{array}$

3.5.4 Conclusions 44

\section{CHAPTER FOUR. The effects of supplementation and serial translocation on} genetic diversity in species with varied life histories

4.1 Abstract 45

4.2 Introduction $\quad 45$

4.2.1 Translocation of skinks to Korapuki Island $\quad 47$

$\begin{array}{ll}4.3 \text { Methods } & 48\end{array}$

4.3.1 Sample collection and microsatellite genotyping 48

4.3.2 Data analysis $\quad 50$

4.4 Results 51

4.4.1 Effects of translocation and supplementation modelled in VORTEX 52

4.4.2 Effects of serial translocation modelled in VORTEX 56

$\begin{array}{ll}4.5 \text { Discussion } & 57\end{array}$

4.5.1 Do models of genetic diversity at neutral loci represent losses of functional diversity? $\quad 57$

4.5.2 How much genetic diversity is enough? 58

4.5.3 Genetic impacts of first- and second- order translocation 59

CHAPTER FIVE. How do reproductive skew and founder group size affect genetic diversity in reintroduced populations?

5.1 Abstract 62

$\begin{array}{ll}5.2 \text { Introduction } & 62\end{array}$

5.2.1 The mating system of a rare reptile 64

5.2.2 Conservation of tuatara by reintroduction 65

$\begin{array}{ll}5.3 \text { Methods } & 66\end{array}$

5.3.1 Microsatellite genotyping $\quad 66$

$\begin{array}{ll}\text { 5.3.2 Data analysis } & 67\end{array}$

$\begin{array}{ll}5.4 \text { Results } & 70\end{array}$ 
$\begin{array}{ll}\text { 5.5 Discussion } & 73\end{array}$

$\begin{array}{ll}\text { 5.5.1 Losses of genetic diversity } & 74\end{array}$

$\begin{array}{ll}\text { 5.5.2 Choice of source population } & 75\end{array}$

5.5.3 Founder group size and composition 75

$\begin{array}{ll}\text { 5.5.4 Reproductive skew } & 76\end{array}$

CHAPTER SIX. Managing genetic diversity in reintroduced populations: Thesis summary and applications

$\begin{array}{ll}6.1 \text { Introduction } & 78\end{array}$

6.2 Summary of findings 78

6.2.1 Assessing genetic diversity for conservation management: a case study of a threatened reptile (Chapter Two) 79

6.2.2 Genetic structure and individual fitness following translocation of a small lizard (Chapter Three) 79

6.2.3 The effects of supplementation and serial translocation on genetic diversity in species with varied life histories (Chapter Four) 80

6.2.4 How do reproductive skew and founder group size affect genetic diversity in reintroduced populations? (Chapter Five) 80

6.3 Implications for conservation management $\quad 81$

6.3.1 Minimising the initial genetic bottleneck 81

6.3.2 Maximising the retention of genetic diversity 82

6.3.3 Management of highly inbred populations 83

6.4 Future directions for research 84

6.4.1 Are the losses of genetic diversity consistent across reintroduced populations? $\quad 84$

6.4.2 How is inbreeding depression manifested in reptiles? 85

6.4.3 How do density-dependent factors operate after reintroduction? 86

6.4.4 How do demography and inbreeding interact to influence population persistence? $\quad 86$

$\begin{array}{ll}6.5 \text { Summary } & 87\end{array}$

$\begin{array}{lr}\text { References } & \mathbf{8 8}\end{array}$

APPENDIX ONE. Alternate escape strategies may provide a means of $\begin{array}{ll}\text { compensation for poor performance ability } & 102\end{array}$

APPENDIX TWO. Taking the plunge: New Zealand's diving skinks 123 


\section{CHAPTER ONE}

\section{Founding events, inbreeding, and their influence on translocated populations: An overview}

\subsection{Introduction}

Translocation is one of the most powerful tools for species conservation and ecosystem restoration. As habitat loss, introduced predators, and disease epidemics threaten species worldwide, the movement of organisms from one area to another provides a means of increasing the numbers and sizes of populations. However, translocations of threatened species generally have low success rates (Griffith et al. 1989; Dodd \& Seigel 1991), and little is known about the causes of failure or the reasons for success (Armstrong \& McLean 1995). Translocation raises a suite of issues surrounding the species' physiology, ecology, genetics, behaviour, and population and community dynamics that may influence the likelihood of success (Dodd \& Seigel 1991; Armstrong \& Seddon 2008). Thus, a strong body of research has emerged to evaluate past translocations and improve the outcomes of future projects (reviewed in Seddon et al. 2007). Understanding the influence of management actions on genetic diversity in translocated populations is identified as a priority for reintroduction biologists (Armstrong \& Seddon 2008). In this thesis, I therefore examine factors that influence inbreeding dynamics and the maintenance of genetic diversity in translocated populations.

\subsection{The importance of genetic diversity}

Genetic diversity is one of the three forms of biodiversity recognised by the World Conservation Union (McNeely et al. 1990), and conserving that diversity has become a cornerstone of modern biology. In small, closed populations, relatives inevitably mate and genetic drift plays a large role in shaping genetic diversity (Allendorf \& Luikart 2007). A sudden reduction in population size, or bottleneck, reduces genetic diversity, which may lead to decreases in fitness (inbreeding depression), increased extinction risk, and reduced evolutionary potential (Frankham 1999; 2005). Although selective forces act to maintain genetic diversity at many functional loci (Aguilar et al. 2004), genetic drift may outweigh the forces of selection. For example, at small population size, drift becomes the 
predominant evolutionary force acting on genetic variability in the fitness-related genes of the major histocompatibility complex (MHC, Miller \& Lambert 2004; Campos et al. 2006). Losses of genetic variation may substantially reduce the ability of a population to respond to novel disease threats, increasing the risk of an epidemic causing a population crash (e.g. Spielman et al. 2004). When populations recover to large population sizes, a loss of genetic variation reduces the potential to respond to selective pressures (Frankham 1999), yet even without additional threats, extinction risk in the wild increases significantly with decreasing genetic diversity (Saccheri et al. 1998).

Inbreeding in the generations following a bottleneck may lead to additional losses of genetic diversity and inbreeding depression. The deleterious consequences of inbreeding in domesticated plants and animals have been recognised since Darwin (Darwin 1896). As inbreeding has negative effects on the health and fitness of individuals (Ralls et al. 1979; Crnokrak \& Roff 1999), it is of great interest to geneticists. In captive populations of ungulates, inbred young show much higher mortality rates than non-inbred young (Ralls et al. 1979). Captive animals are generally well cared for, and inbreeding may have more severe consequences on individuals in the wild (Meagher et al. 2000). Indeed, inbred individuals in wild populations often show signs of inbreeding depression, including a lower probability of survival (e.g. juvenile red deer, Cervus elaphus, Coulson et al. 1999; adult and juvenile pink pigeons, Columba mayeri, Swinnerton et al. 2004; ant queens, Cardiocondyla obscurior, Schrempf et al. 2006; cricket nymphs, Teleogryllus commodus, Drayton et al. 2007; fledgling house sparrows, Passer domesticus, Jensen et al. 2007), lower reproductive output (e.g. song sparrows, Melospiza melodia, Keller 1998; red deer, Slate et al. 2000), or increased susceptibility to disease (e.g. Soay sheep, Ovis aries, Coltman et al. 1999; gazelles, Gazella spp., Cassinello et al. 2001).

Moreover, inbreeding and genetic bottlenecks have negative effects on the stability of populations (Keller \& Waller 2002; O'Grady et al. 2006). Experimental populations of the annual plant Clarkia pulchella with smaller effective population sizes have lower germination and survival rates than populations with larger effective population sizes, making the smaller populations more prone to extinction (Newman \& Pilson 1997). In wild populations of adders (Vipera berus), a small isolated population had lower mean litter size than non-isolated populations (Madsen et al. 1996). Increasing genetic diversity in this isolated population by the addition of conspecifics led to rapid increases in recruitment and population size (Madsen et al. 1999). 
Because of their effects at individual and population levels, inbreeding and reduced genetic diversity directly influence extinction risk in wild populations. In an extreme example, inbreeding explains $26 \%$ of the variation in extinction rate among metapopulations of Glanville fritillary butterflies (Melitaea cinxia, Saccheri et al. 1998). However, it has been suggested that species with a history of small population size and frequent inbreeding (e.g. island endemics) may be less susceptible to the effects of inbreeding because they have been purged of deleterious alleles (Craig 1991). Ancestral inbreeding may expose deleterious alleles to selection, resulting in a decrease in their frequency (Hedrick 1994). Although this view has influenced the management of some species (see Jamieson et al. 2006), purging is unlikely to reduce the negative effects inbreeding depression (Ballou 1997; Frankham 2001, but see Crnokrak \& Barrett 2002). There is little evidence for purging in natural populations, and inbreeding depression has been detected in many island endemics (Jamieson et al. 2006).

Despite the clear links between a loss of genetic diversity, reduced evolutionary potential, and increased extinction risk, it is not entirely clear how much genetic diversity a population can lose before it has a higher risk of extinction or reduced evolutionary potential. In other words, how much genetic diversity must be maintained in order to minimise these effects? As any loss of genetic diversity, particularly due to anthropogenic effects, is considered detrimental, it is generally agreed that the more heterozygosity retained, the better (Franklin \& Frankham 1998; Lynch \& Lande 1998). More tangibly, cited targets for the maintenance of genetic diversity in small populations are usually $90-95 \%$ heterozygosity over 100-200 years (Ralls \& Ballou 1986; Soulé et al. 1986; Allendorf \& Ryman 2002), with the assumption that small losses of genetic diversity should reflect minimal inbreeding and the retention of adaptive potential.

\subsection{Translocations}

Translocation is defined as the movement of living organisms from one area with free release in another (IUCN 1987). Three classes of translocation distinguish between different motivations and population statuses. First, introductions are movements, either intentional or accidental, of organisms outside of their historic range. Second, reintroduction is the intentional movement of an organism into a part of its native range from which it has disappeared or become extirpated in historic times. Third, a 
supplementation is the movement of individuals into a population of conspecifics with the intention of increasing population size.

Reintroductions are increasingly used as a means of species and ecosystem restoration (Lipsey \& Child 2007; Seddon et al. 2007), yet success rates are generally around 50\% for threatened and endangered species (Griffith et al. 1989; Wolf et al. 1996). Reptile and amphibian species worldwide have undergone dramatic declines (Gibbons et al. 2000; Stuart et al. 2004), and translocations of herpetofauna have had lower success rates than translocations of birds and mammals (19\% v. 53\%, respectively, Dodd \& Seigel 1991; Wolf et al. 1996, but see Germano \& Bishop 2009). Reasons for lower success rates may include the unique biophysical constraints imposed by ectothermy, differing biological requirements during different life stages, and disease (Dodd \& Seigel 1991).

New Zealand has the most diverse herpetofauna of any temperate archipelago (Daugherty et al. 1990). Whilst the entire fauna is protected by strict legislation, more than half of the reptile and amphibian species require urgent conservation action (Daugherty et al. 1994; Hitchmough et al. 2007). The arrival of humans in New Zealand led to marked changes in the distribution and abundance of many species (Towns \& Daugherty 1994), leaving about $40 \%$ of all reptile and amphibian species confined mainly or entirely to offshore islands (Daugherty et al. 1994). The introduction of mammalian predators, especially rats (Rattus spp.), has been the primary cause of decline, though habitat alteration and occasional harvest by humans may have contributed to declines of some species (Towns \& Daugherty 1994).

Translocation is one of the most commonly used tools in New Zealand conservation, and over 400 translocations of 40 taxa (primarily birds) were carried out by 1995 (Armstrong \& McLean 1995). The number of translocations has increased dramatically in recent years (Saunders 1995), partially due to the removal of all introduced mammalian predators from about 70 offshore islands, resulting in more than 30,000 ha of pest free habitat (Parkes \& Murphy 2003). Translocation is increasingly used for management of herpetofauna, and over 60 translocations of 30 species have been carried out since 1984 (TP Bell, JM Germano, and KA Miller, unpubl. data). 


\subsection{Genetic diversity in translocated populations}

Despite the known demographic risks and genetic consequences of small population size, reintroduced populations of threatened species are often founded by a small number of individuals (Griffith et al. 1989; Wolf et al. 1996). Founder groups may be small to limit potential impacts on fragile source populations or ecosystems (e.g. Towns \& Ferreira 2001), but the loss of genetic diversity combined with inbreeding depression in a small reintroduced population could reduce the probability of establishment and persistence (Armstrong \& Seddon 2008). Therefore, maximising genetic diversity in reintroduced populations is important for both short- and long-term success (Fitzsimmons et al. 1997; Allendorf \& Luikart 2007; Armstrong \& Seddon 2008). In New Zealand, founder groups are often very small (less than 30 individuals, Lovegrove 1996; Towns \& Ferreira 2001; Jamieson et al. 2003), and the neglect of the potentially serious consequences of inbreeding depression in some recovery programs has generated criticism (Jamieson et al. 2006).

The maintenance of genetic diversity in a translocated population will be affected differentially by several factors. First, small founder groups may cause a genetic bottleneck, and small effective population sizes promote the loss of genetic diversity. Second, intentionally choosing diverse founders or mixing founders from several sources may promote higher genetic diversity. Third, increasing population size generally promotes the maintenance of genetic diversity. Lastly, many mating systems reduce effective population size and may cause a loss of genetic diversity, but largely overlapping generations may minimise this effect. Reintroduced populations often retain only a small proportion of the genetic variation of their sources at both neutral and functional loci (Williams et al. 2000; Williams et al. 2002; Miller \& Lambert 2004). This reduction in diversity is intensified by small founding populations, and the genetic signatures of reintroduction events may be accentuated by population isolation (Latch and Rhodes 2005).

The empirical evidence that reintroductions cause genetic bottlenecks is equivocal. Reintroductions of threatened species are typically sourced from small populations or those that have themselves been through a bottleneck, and the signatures of a bottleneck in a source population may make it hard to distinguish the direct effects of translocation. Reduced heterozygosity and allelic diversity in reintroduced populations 
are well documented (Fitzsimmons et al. 1997; Williams et al. 2000; Swanson et al. 2006), yet other signatures of genetic bottlenecks are not commonly described. Although translocated populations of Alpine ibex (Capra ibex) showed multiple signatures of genetic bottlenecks, the source population showed the same signals, and a population bottleneck in the source prior to translocation had a greater effect on the patterns of genetic diversity (Maudet et al. 2002). When multiple source populations were used for the reintroduction of American martens (Martes americana), no evidence of a genetic bottleneck was detectable (Swanson et al. 2006). Further, genetic bottlenecks were only associated with a translocated population of elk (Cervus elaphus) that remained smaller than 50 individuals for 50 years (due to legal harvest), and not with a larger translocation that expanded rapidly (Williams et al. 2002).

Apart from theoretical concerns over maintenance of diversity during translocations and empirical evidence showing reduced genetic diversity after translocations, there is little evidence of decreased fitness in reintroduced populations. The correlation between a loss of genetic diversity during reintroduction and increased extinction risk is even less clear. Reintroduced takahe (Porphyrio hochstetteri) show signs of environmentally-induced inbreeding depression (Jamieson and Ryan 2000), but high levels of inbreeding in the source population prior to translocation make assessment of inbreeding depression difficult (Jamieson et al. 2003). Reduced juvenile survival was only evident in highly inbred North Island robins (Petroica longipes), which became rare after the population expanded (Jamieson et al. 2007). Further, inbreeding depression may have only minimal effects on population establishment. Even when egg failure rates are tripled, extinction risk increases by only 33\% for reintroductions of South Island robins (Petroica australis) founded with as few as four individuals (Taylor et al. 2005). The probability of extinction was negligible in a species with a higher finite rate of population growth (South Island saddlebacks, Philesturnus carunculatus, Taylor et al. 2005). However, the more insidious consequences of a loss of genetic variation and thus adaptive potential may have a greater effect on the long-term persistence of these populations (Frankham 1999; Spielman et al. 2004).

\subsection{Thesis structure}

This aim of this thesis is to advance the current understanding of the factors that influence inbreeding dynamics and the long-term maintenance of genetic diversity in 
reintroduced populations. Understanding and predicting how management actions affect genetic diversity in translocated populations has been identified as a priority both for species recovery (e.g. Towns 1999) and in the broader field of reintroduction biology (Armstrong \& Seddon 2008). The results of this thesis will therefore provide information to managers and researchers that improves translocation planning and the understanding of how management practices affect the adaptive potential of populations. The questions underpinning this thesis are:

1) How can genetic diversity in founder groups be maximised?

2) Can the negative consequences of inbreeding be easily detected in cryptic species?

3) How do life history traits and the mating system of a species affect long-term patterns of genetic diversity?

In order to realistically evaluate options for the genetic management of threatened species, it is essential to appreciate the conservation climate in which management decisions are based. Thus in Chapter Two, I present a case study that demonstrates the difficulty in using genetic data to manage threatened species. Using data from the three remaining natural populations of Whitaker's skink (Cyclodina whitakeri), I explore the genetic relationships among populations. I then evaluate options for a reintroduction of Whitaker's skink from captivity to the wild, including potential for inter-population hybridisation. Chapter Two has been published as:

Miller KA, DG Chapple, DR Towns, PA Ritchie, and NJ Nelson (2009) Assessing genetic diversity for conservation management: a case study of a threatened reptile. Anim Conserv 12: 163-171

Determining the best strategies for the long-term maintenance of genetic diversity after translocation first requires knowledge of how to minimise the initial genetic bottleneck. Chapter Three delves in depth into the changes in genetic structure of translocated populations in the first few generations following release. I use a well-studied reintroduced population of the egg-laying skink (Oligosoma suteri) to provide a detailed picture of how genetic drift affects populations and how the release of gravid females influences effective population size. I also investigate the dynamics of inbreeding in this population and how inbreeding influences fitness, by examining performance-based surrogates of fitness. Chapter Three has been submitted to Conservation Genetics as: 
Miller KA, DR Towns, PA Ritchie, FW Allendorf, and NJ Nelson (in review) Genetic structure and individual fitness following translocation of a small lizard. Conserv Genet

Chapters Four and Five build on the previous chapters by examining the factors that influence how well genetic diversity will be maintained over 10 generations after translocation. In Chapter Four, I compare how well genetic diversity was maintained in founder groups during the reintroduction of three species of skink, and over 14-18 years following release. I then model how the different life history traits of these species affect the maintenance of genetic diversity in these reintroduced populations. Lastly, I evaluate how proposed strategies for management (supplementation and serial translocation) may minimise or accelerate the loss of genetic diversity in these populations. This chapter is currently in preparation for submission to Conservation Biology:

Miller KA, NJ Nelson, FW Allendorf, and DR Towns (in prep) The effects of supplementation and serial translocation on genetic diversity in species with varied life histories

In the final data chapter, Chapter Five, I focus on how the mating system of a species may influence the maintenance of genetic diversity, how that system may change after translocation, and how different founder groups may affect long-term genetic trends. I use populations of tuatara (Sphendon) translocated from both high- and low-diversity wild populations to examine the losses of genetic diversity in founder groups of different sizes. I then model how variance in male reproductive success influences the maintenance of genetic diversity over 10 generations. A version of Chapter Five has been published as:

Miller KA, NJ Nelson, HG Smith, and JA Moore (in press) How do reproductive skew and founder group size affect genetic diversity in reintroduced populations? Mol Ecol

This thesis is written as a series of independent manuscripts. As such, there may be some repetition in the introductions to each chapter. Chapters Two through Five include an introduction to the specific aims and hypotheses, as well as a discussion of the important findings in a broader context. Finally, Chapter Six provides a discussion on the more general implications of the thesis for conservation management and future directions for research. 


\subsection{Statement of authorship}

Various collaborators have earned co-authorship on individual manuscripts included in this thesis, and I outline their specific contributions below. For all manuscripts, I conceived the questions, conceptualised and conducted the analyses, and wrote each draft. I also collected all samples in the field and conducted all of the lab work, unless otherwise stated. All co-authors provided comments on earlier drafts.

Nicky Nelson and Dave Towns recommended appropriate model systems and helped in planning the projects, including permitting, field work, and travel. They both gave extensive insight into the issues surrounding conservation management in New Zealand and the implications of my results. Additionally, Nicky Nelson gave statistical advice for Chapter Three and had extensive discussions with me about appropriate input parameters for Chapter Five. Dave Towns provided skink tissue samples collected between 1988 and 1999, advised me on appropriate input parameters for models in Chapter Four, and provided unpublished data on survival of individuals after release.

Fred Allendorf and Pete Ritchie provided advice during the planning of the projects, including appropriate study design, and advised on the best analytical approach. Fred Allendorf also recommended additional analyses to strengthen the manuscripts and discussed most relevant aspects of population modelling given empirical results.

Dave Chapple provided $5 \mathrm{mtDNA}$ sequences for Chapter Two, helped in using some of the programmes, and discussed interpretation of the results. For Chapter Five, Hayden Smith wrote a short computer program to calculate allelic diversity from raw genotypic output from VORTEX. Jen Moore collected tuatara DNA samples from Stephens Island for Chapter Five, conducted lab work on those animals, and provided advice on accurately modelling the mating system of tuatara. 


\section{CHAPTER TWO}

\section{Assessing genetic diversity for conservation management: a case study of a threatened reptile}

\subsection{Abstract}

The consequences of inbreeding in small isolated populations are well documented, yet populations are often managed in isolation to avoid irreversibly mixing genetic lineages and to maintain the historic integrity of each population. Three remaining populations of Whitaker's skink (Cyclodina whitakeri) in New Zealand, remnants of a once wider distribution, illustrate the conflict between this genetic goal (separate management of populations) with the more tangible and immediate threats of small population size and inbreeding. Middle and Castle Islands harbour populations of $C$. whitakeri and have been separated from each other and from the mainland for $\sim 10,000$ years. The single mainland population at Pukerua Bay is extremely small, declining, and deemed a high priority for management. We sequenced a 550 bp region of mitochondrial DNA (ND2) and genotyped animals from all three populations at 13 microsatellite loci. The population of C. whitakeri at Pukerua Bay showed marked differences from the island populations at both mtDNA (unique, fixed haplotype) and microsatellite loci $\left(F_{S T} \sim 0.20\right)$, and private alleles were detected at a high frequency ( $24 \%$ of all alleles). However, we attribute this pattern to an historic genetic gradient coupled with rapid genetic drift. Further, animals in captivity show genetic signatures of both Pukerua Bay and island populations, despite the goal to maintain a pure Pukerua Bay stock. The mixed genetic stock in captivity provides an opportunity for the addition of skinks from Middle Island to evaluate the risks of further population hybridisation, including the disruption of potential local adaptation, while mitigating the risks of inbreeding.

\subsection{Introduction}

Balancing the immediate demographic threats to a population with long-term goals for genetic management can be a difficult and daunting task. The factors that cause population declines (e.g. disease or introduced predators) are often severe and pose a more tangible and immediate threat of extinction than the effects of inbreeding (Thorne 
\& Williams 1988; La Marca et al. 2005; Tocher \& Norbury 2005). Indeed, genetic factors such as inbreeding depression will have relatively little impact while populations are rapidly declining due to high predation rates or disease (Jamieson 2007). Yet during population recovery, inbreeding depression may have a large impact on population growth rates and extinction risk (Frankham 2005; O'Grady et al. 2006).

Inbreeding is inevitable in small closed populations (Allendorf \& Luikart 2007); thus management of threatened species in isolation may impose unnecessary inbreeding depression. Recovery programmes in New Zealand have been criticised for neglecting the potential problems of inbreeding (Jamieson et al. 2006), particularly when genetic exchange among populations could minimise these effects (Allendorf 2001). Interpopulation hybridisation can result in an increase in fitness (Hedrick 1995; Westemeier et al. 1998; Madsen et al. 1999). Alternately, hybridisation may reduce fitness in first, second, or later generation hybrids (Tallmon et al. 2004). Empirical evidence for inbreeding depression outweighs that of outbreeding depression (Saccheri et al. 1998; Crnokrak \& Roff 1999), but the available information shows that the effects of outbreeding are severe (Marr et al. 2002; Edmands 2007 for review). In the case of threatened species, where decisions about management are both difficult and central to species survival, it becomes particularly apparent that information on the genetic differences among populations is important for adequate management.

New Zealand's lizard fauna may be the most diverse of any temperate archipelago (Daugherty et al. 1990), but human colonisation led to radical changes in the abundance and distribution of many species. The primary cause of decline has been introduced mammalian predators, especially rats (Rattus spp., Towns \& Daugherty 1994). At least 40\% of New Zealand's herpetofauna is now restricted mainly or entirely to predator-free offshore islands (Daugherty et al. 1994). The ground-dwelling, relatively large-bodied, nocturnal skinks in the genus Cyclodina (Scincidae) are particularly susceptible to the effects of introduced mammals. Seven of the 10 species of Cyclodina have been effectively eliminated from mainland New Zealand by introduced predators (Daugherty et al. 1994; Towns \& Daugherty 1994; Chapple et al. 2008).

Whitaker's skink (Cyclodina whitakeri) is an excellent example of the complexity of applying genetic data to the management of fragmented populations. Though once distributed across the North Island (Worthy 1987), C. whitakeri now naturally occurs on 
only two small islands and one mainland site. It is listed as "vulnerable" (D1 + D2) by the World Conservation Union (2007), and its national threat status was heightened in 2007 by the New Zealand Department of Conservation (DOC) because of population declines in the previous three years (Hitchmough et al. 2007).

The three remaining natural populations of $C$. whitakeri total $<20$ ha of habitat (Towns 1992b) and are separated by up to $500 \mathrm{~km}$ (Figure 2.1). Two island populations are found on predator-free Middle and Castle Islands, and a single mainland population is located at Pukerua Bay. The largest population (estimated at 1,300 - 12,000 individuals, Southey 1985 ) on Middle Island (13 ha) is considered stable. Castle Island ( 3 ha) is privately owned and thus not managed by DOC. The skink population on Castle Island is not given a high level of security (Towns 1992b; 1999), but is presumed stable because mammalian predators are not present. Surveys have not been conducted in the last 15 years because of limited access to the island. The mainland population of $C$. whitakeri at Pukerua Bay is confined to $<1$ ha of habitat and persists only in deep boulder banks (Towns 1992b; Towns \& Elliott 1996). This population declined drastically during a 23-year monitoring period between 1984 and 2006, presumably due to continued predation by introduced mammals (Hoare et al. 2007).

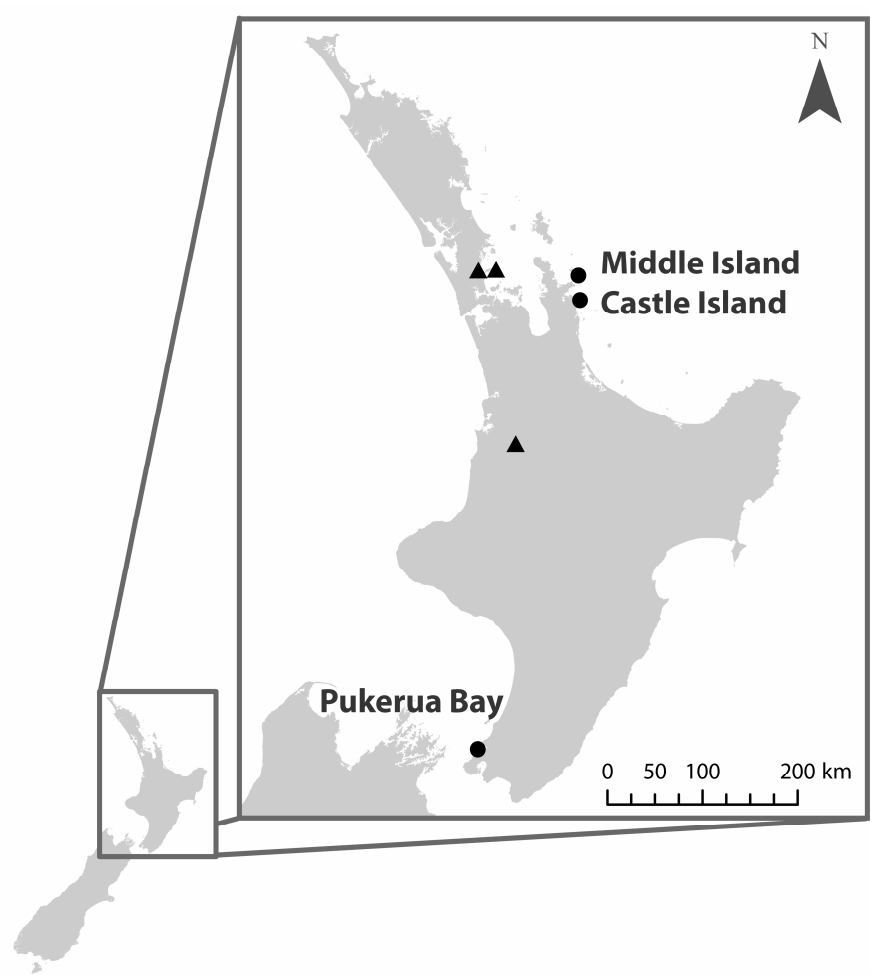

Figure 2.1 Map of Cyclodina whitakeri distribution. Closed circles indicate extant populations; triangles indicate locations of subfossil remains (from Worthy 1987). 
The genetic relationships among populations of $C$. whitakeri are not well understood. Middle and Castle Islands have been separated from each other and the mainland for 10,000 years (Hayward 1986; Towns 1994). Lizards are generally good colonisers of oceanic islands (i.e. dispersing over saltwater, Thomas \& Whitaker 1996; Censky et al. 1998), but $C$. whitakeri has one of the highest rates of cutaneous water loss among lizards and is physiologically intolerant to dehydrating conditions (Cree \& Daugherty 1991). Contemporary gene flow (by dispersal) between populations is unlikely, but the three populations show no differentiation at allozyme loci (Towns \& Daugherty 1994). Shallow divergence in mtDNA suggests that gene flow across the range of $C$. whitakeri persisted until the late Pleistocene (Chapple et al. 2008), and this estimate is consistent with the separation of the island populations from the mainland.

Pukerua Bay is a "Key Place" in the Wellington Conservancy's Conservation Management Strategy (Anon 1996); management of this reserve is targeted at the protection of $C$. whitakeri. Additionally, management of this population and translocation of animals from the mainland to predator-free island sites are high priorities for the species (Miskelly 1999; Towns 1999). However, the current capture rate of $C$. whitakeri at Pukerua Bay (0.03 per 100 trap nights) is insufficient to produce a propagule for translocation; only three animals have been captured since 2001. Construction of a predator-proof fence is the most effective way to protect mainland populations from introduced predators, and would provide the best chance of capturing C. whitakeri (Hoare et al. 2007). However, this measure has several challenges, including high start-up costs. If the population at Pukerua Bay is genetically distinct, it may influence whether this management action is deemed a priority.

Skinks from Pukerua Bay are held in captivity with the intention of using captive-bred animals as founders of a new population on a predator-free island. All 12 F1 offspring in the programme have been produced by a single pair: a female from Pukerua Bay and a male (of presumed Pukerua Bay origin) that have been part of the breeding programme for $\sim 10$ years. The male was taken into the captive breeding programme after a private breeder abandoned his collection, and confirmation of its origin was not obtained. A second male, taken into captivity from Pukerua Bay by DOC in 2005, has sired offspring from $F 1$ females. 
Here we evaluate both mitochondrial and microsatellite diversity for the three populations of $C$. whitakeri to inform conservation management. We aim to determine conservation units under traditional definitions, and assess the validity of those units given the challenges faced in managing C. whitakeri and the limitations encountered when sampling is constrained by current population status. Lastly, we aim to resolve the origin of the captive male (of presumed Pukerua Bay origin) to make recommendations for the future management of the captive population.

\subsection{Methods}

\subsubsection{Sample collection and DNA extraction}

We sampled 51 animals: 44 from Middle Island (preserved specimens and field collected), all available samples from Castle Island (one preserved specimen) and Pukerua Bay (three preserved specimens and two animals in captivity), and the captive male of presumed Pukerua Bay origin. Tail or toe samples ( $3 \mathrm{~mm}$ ) from each animal were stored in $70 \%$ ethanol. Total genomic DNA extraction was performed using a standard proteinase $K$ phenol-chloroform protocol (Sambrook et al. 1989) followed by ethanol precipitation. DNA was quantified using a NanoDrop ${ }^{\circledR}$ ND-1000 Spectrophotometer at $260 \mathrm{~nm}$.

\subsubsection{Mitochondrial DNA sequencing}

We sequenced a $\sim 600 \mathrm{bp}$ portion of the mitochondrial gene ND2, a region commonly selected for work at comparable taxonomic levels in squamate reptiles (Keogh et al. 2005; Hare et al. 2008) and previously used to investigate the phylogeography of the genus Cyclodina (Chapple et al. 2008). We attempted to sequence all 51 samples, but 10 samples from Middle Island failed to amplify. The primers used to amplify and sequence ND2 were L4437 (Macey et al. 1997) and ND2r102 (Sadlier et al. 2004). PCR and sequencing were conducted as outlined in Greaves et al. (2007). Sequence data were edited using CONTIGEXPRESS 9.1.0 (Invitrogen) and aligned using the default parameters in CLUSTAL X (Thompson et al. 1997). The edited alignment comprised the 550 bp sequence reported by Chapple et al. (2008). 


\subsubsection{Microsatellite genotyping}

All individuals were genotyped at 13 microsatellite loci (Berry et al. 2003) adapted with $5^{\prime}$ M-13 tags (TGTAAAACGACGGCCAGT; Schuelke 2000). PCR was performed in $15 \mu \mathrm{L}$ reactions containing: PCR buffer (Invitrogen), $2.5 \mathrm{mM} \mathrm{MgCl} 2,0.4 \mu \mathrm{g} / \mathrm{mL}$ bovine serum albumin, $0.2 \mathrm{mM}$ each dNTP, $0.375 \mathrm{U}$ Taq DNA polymerase (Invitrogen), and $~ 35 \mathrm{ng}$ genomic DNA. Locus-specific forward primer concentrations were between 0.25 and $0.53 \mu \mathrm{M}$; fluorescently-labelled M-13 universal primers (Schuelke 2000) were at equal concentrations with reverse primers. PCR was carried out on an Eppendorf Mastercycler thermocycler. The PCR profile consisted of one cycle of $94^{\circ} \mathrm{C}$ for $3 \mathrm{~min}, 30$ cycles of $\left(94^{\circ} \mathrm{C}\right.$ for $30 \mathrm{~s}, 57^{\circ} \mathrm{C}$ for $45 \mathrm{~s}, 72{ }^{\circ} \mathrm{C}$ for $\left.45 \mathrm{~s}\right)$, eight cycles of $\left(94^{\circ} \mathrm{C}\right.$ for $30 \mathrm{~s}, 53^{\circ} \mathrm{C}$ for $45 \mathrm{~s}, 72{ }^{\circ} \mathrm{C}$ for $30 \mathrm{~s}$ ), and a final extension at $72{ }^{\circ} \mathrm{C}$ for $15 \mathrm{~min}$. PCR products were pooled for genotyping on an ABI3730 Genetic Analyzer (Applied Biosystems, Inc.). Allele sizes were scored manually by KAM using GENEMAPPER 3.7 (Applied Biosystems, Inc.).

\subsubsection{Data analysis}

The captive male of presumed Pukerua Bay origin was removed from all analyses except for the population assignment test. The Castle Island individual was removed for all statistical microsatellite analyses because only one sample was available.

Haplotype diversity $(h)$ and nucleotide diversity $(\pi)$ were calculated in DNASP 4.10.9 (Rozas et al. 2003), and uncorrected mtDNA sequence divergence was calculated in MEGA 3.1 (Kumar et al. 2004). A haplotype network was created in TCS 1.21 (Clement et al. 2000).

We calculated the frequency of null alleles in FREENA (Chapuis \& Estoup 2007), and found that two loci (Oligr3 and Oligr13) had a high frequency of null alleles in the Middle Island population (0.19 and 0.08, respectively). Two loci (Oligr2 and Oligr17) had a high frequency of null alleles in Pukerua Bay (0.29 and 0.15, respectively). Null alleles affect estimates of population differentiation by reducing intra-population genetic diversity (Paetkau \& Strobeck 1995), and $\mathrm{F}_{\mathrm{ST}}$ is generally overestimated in the presence of null alleles (Chapuis \& Estoup 2007). We therefore excluded Oligr3 and Oligr13 from analyses of population differentiation and assignment tests. We did not exclude Oligr2 or Oligr17 from any analyses, because null alleles were not detected in the Middle Island sample, 
and we could not rule out that detection of null alleles may have been related to the sample size of the Pukerua Bay population.

Departures from Hardy-Weinberg proportions (Guo \& Thompson 1992) and FST (Weir \& Cockerham 1984) were calculated in GENEPOP 4.0 (Rousset 2008). Tests of significance were combined over all loci using Fisher's combined probability test. We also conducted an AMOVA using GenAlEX 6.1 (Peakall \& Smouse 2006). Using FSTAT 2.9 (Goudet 1995), we calculated allelic richness (number of alleles corrected for sample size) at all 13 loci in the Middle Island population based on a sample of five individuals (i.e. the sample size from Pukerua Bay).

Assignment/exclusion tests were conducted in GENECLASS2 (Piry et al. 2004) which assigns individuals to a population and computes the probability that the individual's multilocus genotype would be encountered in each reference population. Probabilities were calculated using the Monte-Carlo resampling method of Paetkau et al. (2004) to reflect the sampling variance associated with the dataset. An assignment test was run on all individuals from our reference populations (Middle Island and Pukerua Bay) to assess the power of our dataset. A second test was run to assign the captive male of presumed Pukerua Bay origin to one of the two reference populations.

\subsection{Results}

\subsubsection{MtDNA variation}

We obtained 41 mtDNA sequences and observed 11 ND2 haplotypes in total (GenBank Accession numbers: EU852568- EU852578). All Pukerua Bay animals shared a single haplotype that was not found in either Middle Island or Castle Island samples. The Castle Island animal shared a haplotype with Middle Island. Overall haplotype diversity was high $(0.89 \pm 0.003$, Table 2.1$)$, as was diversity within Middle Island $(0.88 \pm 0.005)$. The relationships among haplotypes are shown in Figure 2.2. The captive male of presumed Pukerua Bay origin shared a haplotype with both Middle and Castle Islands.

Geographic structuring among populations was minor (mean sequence divergence across all samples $=0.87 \% \pm 0.23$ ). Sequence divergence between Pukerua Bay and the island populations (Middle Island: $1.14 \% \pm 0.36$, Castle Island: $0.36 \% \pm 0.25$ ) were not 
greater than between the island populations (Middle to Castle Island $=1.05 \% \pm 0.33$ ) or the mean sequence divergence within Middle Island $(0.80 \% \pm 0.20)$.

Table 2.1 Variation in mtDNA across the three populations of Cyclodina whitakeri. Overall haplotype diversity was high, but was mostly influenced by intra-population diversity in Middle Island.

\begin{tabular}{ccccc}
\hline & Sample Size & $\begin{array}{c}\text { Number of } \\
\text { Haplotypes }\end{array}$ & $\begin{array}{c}\text { Haplotype } \\
\text { diversity }(h)\end{array}$ & $\begin{array}{c}\text { Nucleotide } \\
\text { diversity }(\pi)\end{array}$ \\
\hline Middle Island & 34 & 10 & $0.88 \pm 0.005$ & $0.79 \%$ \\
Pukerua Bay & 5 & 1 & 0.0 & 0.0 \\
Castle Island & 1 & 1 & -- & - \\
Total & $\mathbf{4 0}$ & $\mathbf{1 1}$ & $\mathbf{0 . 8 9} \pm \mathbf{0 . 0 0 3}$ & $\mathbf{0 . 8 7 \%}$ \\
\hline
\end{tabular}

\subsubsection{Microsatellite variation within populations}

Twelve of 13 microsatellite loci surveyed were polymorphic (2-18 alleles each, Table 2.2). After removal of Oligr3 and Oligr13 (loci with high frequencies of null alleles), both Middle Island and Pukerua Bay populations were in Hardy-Weinberg equilibrium ( $p=0.17$ and $p=0.08$, respectively). Only one locus (Oligr2) in Pukerua Bay deviated significantly from Hardy-Weinberg proportions ( $p=0.02$ after sequential Bonferroni correction).

Genetic differentiation between Middle Island and Pukerua Bay was high ( $\left.F_{S T}=0.19\right)$ across 11 loci. The estimate of differentiation between populations was higher using an AMOVA framework, which considers relationships between alleles $\left(\Phi_{\mathrm{PT}}=0.27, p<0.001\right)$. The population at Pukerua Bay had fewer alleles per locus than the population on Middle Island, even after correction for discrepancies in sample sizes (Table 2.2).

Private alleles were only labelled in Pukerua Bay and Castle Island populations (Table 2.2), as sample sizes were unequal between populations, and the detection of alleles is highly dependent on sample size. Five loci had a total of eight private alleles in Pukerua Bay, representing $24.2 \%$ of the total alleles found in Pukerua Bay samples across all 13 loci (Table 2.2). All Pukerua Bay animals had between one and four private alleles. The single sample from Castle Island had private alleles at 4 loci, representing $19 \%$ of the total alleles found across 13 loci (Table 2.2). 
All Middle Island and Pukerua Bay animals were correctly assigned to their population of origin $(p<0.04)$. The captive male of presumed Pukerua Bay origin was assigned to Middle Island, and Pukerua Bay was excluded as a possible origin $(p<0.001)$.

Middle Island

Castle Island

- Pukerua Bay

nd

3

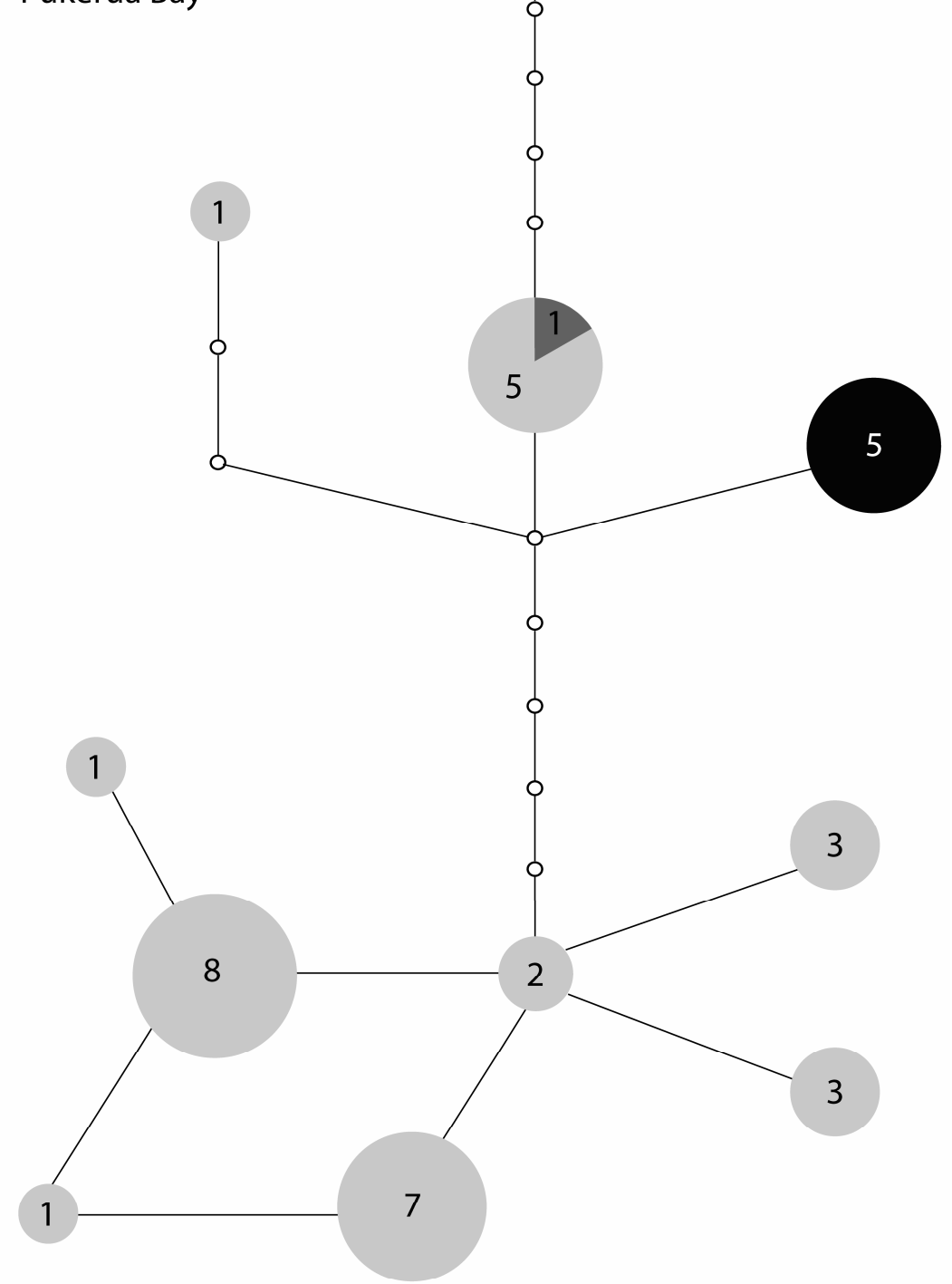

Figure 2.2 Network showing the relationship among haplotypes of Cyclodina whitakeri. Each circle represents one haplotype, and sizes indicate the number of samples (inset) with each haplotype. Each line represents one base pair change between sequences. 


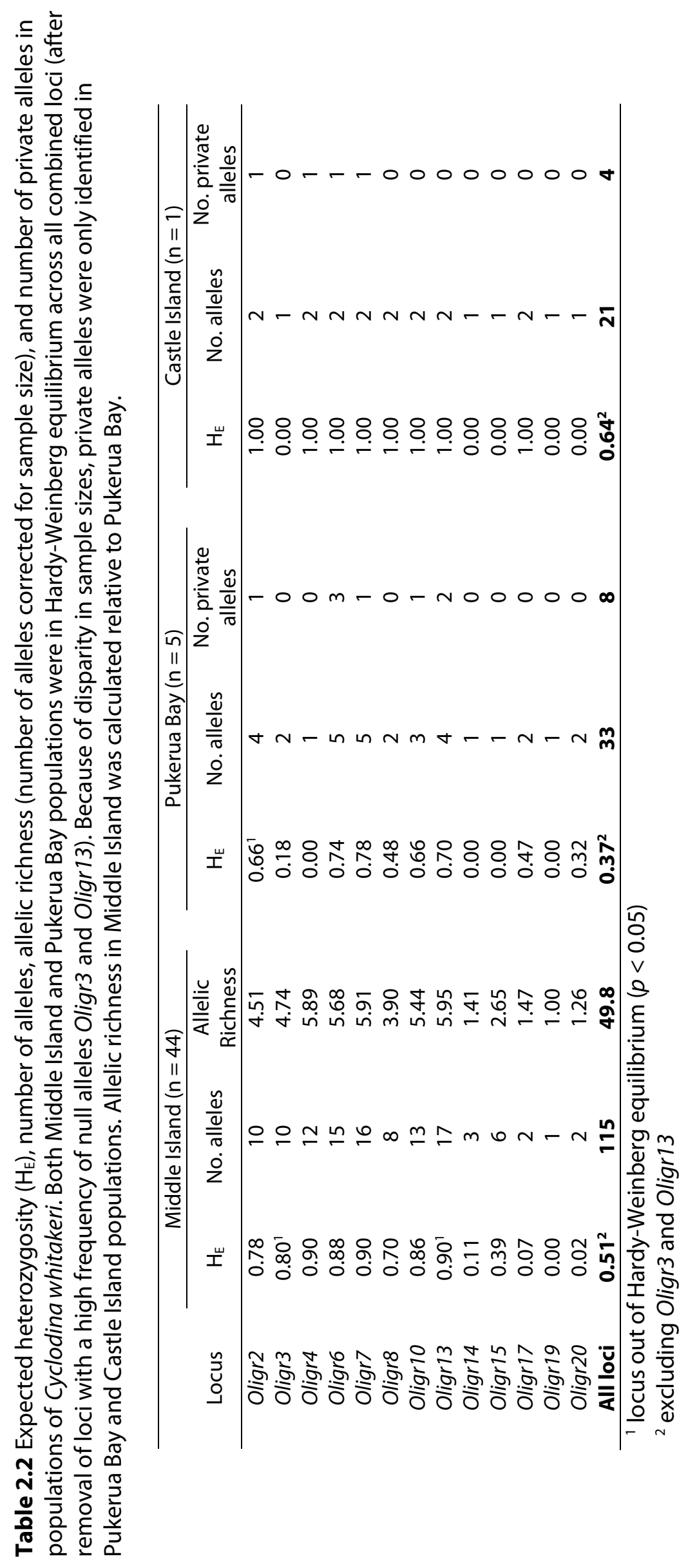




\subsection{Discussion}

Patterns of genetic diversity at both mtDNA and microsatellites indicate that the island and Pukerua Bay populations of $C$. whitakeri are strongly differentiated. When threatened species are reduced to very few natural populations, we must consider the risks of managing discrete populations separately against the alternative of managing them together, particularly when one population is disproportionately vulnerable to extinction. Only the Middle Island population of C. whitakeri is stable (Towns 1999), and the Pukerua Bay population is highly vulnerable.

Populations are often labelled as Evolutionary Significant Units (ESUs) when they are reciprocally monophyletic at mtDNA and show significant divergences at nuclear loci (Moritz 1994). This definition is used most frequently as it is simple to implement (de Guia \& Saitoh 2007), but small bottlenecked populations are more likely to be identified as ESUs when only neutral markers are considered (Allendorf \& Luikart 2007). The island and Pukerua Bay populations qualify as separate ESUs under this definition, but recent population declines limit its applicability. More robust ESU definitions use data on nonmolecular traits, including adaptive differences and ecological exchangeability (Waples 1991; Crandall et al. 2000). Other authors suggest that both the specific management objectives for and the anthropogenic risks faced by the species must be considered (Taylor \& Dizon 1999), and that no single definition will work in all cases (Fraser \& Bernatchez 2001).

When populations are extremely small and/or rare, it is difficult to obtain the required ecological information to evaluate more robust definitions of ESUs. However, local adaptation, particularly with respect to temperature in ectotherms, is possible when populations survive in atypical habitats. Cyclodina whitakeri typically live in seabird burrows (Southey 1985), but occupy deep boulder banks at Pukerua Bay. Seabird burrows maintain stable temperatures $\left(18-19^{\circ} \mathrm{C}\right)$ and high humidity $(>90 \%$, Towns $1992 \mathrm{a})$, but are not found at Pukerua Bay, as seabirds are also vulnerable to mammalian predators. Although the use of boulder bank habitat most likely reflects a response to mammalian predators (Towns \& Elliott 1996; Hoare et al. 2007), animals at Pukerua Bay may have adapted to thermal conditions. Temperature and humidity are major physiological constraints for C. whitakeri, and temperatures at Pukerua Bay are relatively low, as Pukerua Bay lies $\sim 500 \mathrm{~km}$ south of the two island populations. Temperature would be a 
major selective force for $C$. whitakeri, and animals in captivity have died during winter frosts typical in the Pukerua Bay region. It is possible that the population is locally adapted to conditions at Pukerua Bay, but without clear evidence of adaptive divergence, the island populations and Pukerua Bay should be treated as a single unit of conservation (Crandall et al. 2000).

Whilst the population of $C$. whitakeri at Pukerua Bay shows differences from the island populations at both mitochondrial DNA and microsatellite loci, this pattern is most likely a product of an historic genetic gradient coupled with strong genetic drift. Populations of C. whitakeri have declined since the introduction of mammalian predators. The three remnant populations are at opposite ends of the species historic range (Worthy 1987); historic population structure due to isolation by distance could partially explain the relatively large proportion of private alleles ( $24.2 \%$ of all alleles found in Pukerua Bay, Table 2.2) and a unique mtDNA haplotype. With a population size almost certainly smaller than 100 individuals (extrapolated from Towns \& Elliott 1996 and Hoare et al. 2007), the population at Pukerua Bay is clearly in a demographic bottleneck. Genetic diversity is reduced at both mtDNA and microsatellites in the Pukerua Bay population, indicating that the genetic differences between Middle Island and Pukerua Bay skinks $\left(F_{S T}=0.19\right.$ $0.27)$ could be due to rapid genetic drift. Differentiation is large and significant, but measures of $\mathrm{F}_{S T}$ do not necessarily reflect variation in quantitative traits (Reed \& Frankham 2001, but see Merilä \& Crnokrak 2001). However, the effects of genetic drift may supersede adaptation in cases of severe bottlenecks (Miller et al. 2008).

The recent decline of $C$. whitakeri on the mainland has most likely produced the observed pattern of mitochondrial diversity. Patterns of mitochondrial diversity in two widespread Cyclodina species (C. aenea and C. ornata, Chapple et al. 2008), whose distribution reflect the historic distribution of $C$. whitakeri (Worthy 1987) support this assertion. These species inhabit similar habitat (lowland forest) and may provide insight into historic relationships among C. whitakeri populations. Populations of C. aenea from the lower North Island (including Pukerua Bay) and the Mercury Island group (the group containing Middle Island) belong to a single clade with sequence divergence equal to that seen across populations of $C$. whitakeri, yet populations of $C$. aenea within the Mercury Island group belong to two different clades (Chapple et al. 2008). Populations of $C$. ornata from the lower North Island also group with a northern population (Chapple et al. 2008). Further, the greatest source of mtDNA diversity was found north of the Mercury Islands in both $C$. 
aenea and $C$. ornata, indicating that perhaps the most divergent populations of $C$. whitakeri were once also found in that range.

Both mtDNA and microsatellite data confirm that the captive male of presumed Pukerua Bay origin likely belongs to one of the island populations. We cannot conclude from which of these populations he originated, as this male shared an mtDNA haplotype with both Middle and Castle Islands. Due to small sample size from Castle Island, it is currently not possible to use nuclear DNA to distinguish between Middle and Castle Islands as the origin of the captive male. However, the high frequency of private alleles in the single sample from Castle Island (19\% of all alleles) indicates that further sampling is required to determine the extent of genetic differentiation of this population.

Evaluating the risks of intentional population hybridisation is a complex task (Edmands 2007), and genetic risks must be considered along with many others. The unique set of factors influencing management of a species will have an impact on whether intraspecific hybridisation is a viable option for management (Westemeier et al. 1998; Ebert et al. 2002; Gleeson et al. 2007). Inbreeding depression can be difficult to demonstrate in cryptic species. However, realistic levels of inbreeding depression influence extinction risks in natural populations, and inappropriate recovery strategies may be implemented if genetic factors are ignored (Frankham 2005; O'Grady et al. 2006). Isolated relict populations cannot be conserved indefinitely with small size and hybridisation may be a viable option for recovery (Westemeier et al. 1998). After introduction of genetic diversity from three populations to a small isolated population in Illinois, greater prairie chicken (Tympanuchus cupido pinnatus) egg fertility increased by up to $25 \%$ (Westemeier et al. 1998). Florida panthers (Puma concolor coryi) were hybridised with the subspecies from Texas (P. c. stanleyana) in 1995 with multiple benefits. Survival of hybrid kittens was three times higher than that of purebred kittens (Pimm et al. 2006) and no hybrid animals suffered from cryptorchidism, a condition common in purebred Florida panthers that may reduce fertility (Mansfield \& Land 2002). Similarly, the release of adders (Vipera berus) from two non-isolated populations into a population suffering from inbreeding depression led to a rapid decrease in the proportion of stillborn young (Madsen et al. 1996) and an increase in recruitment (Madsen et al. 1999).

When species are extremely rare, decisions about genetic management are often limited by small sample sizes. An adaptive management approach may be valuable in these 
situations. For C. whitakeri, genetic data indicate that management efforts should focus on implementing strategies to increase the size of the Pukerua Bay colony in captivity and on sampling individuals from Castle Island. Further, this approach should be used to evaluate the effects of hybridisation in the captive colony. An adaptive management approach would require commitment to continued genetic monitoring, but would reduce uncertainty over time by accruing the data needed to improve future management.

\subsubsection{Management implications}

Current management is aimed at maintaining a pure Pukerua Bay population in captivity for translocation (Miskelly 1999). However, the sire of all $12 \mathrm{~F} 1$ offspring in the programme originated from an island population (this study). In light of our genetic data, there are four options for continued management of this population: 1) continuation of the programme "as is", accepting that population hybridisation has occurred; 2) restarting the captive programme using only Pukerua Bay animals; 3 ) maintaining both pure and hybrid populations, while adding more animals from Middle Island (adaptive approach); or 4) terminating the captive breeding programme and transferring captive animals to a predator-free island. All of these options pose substantial risks of inbreeding depression if new animals are not introduced from the wild. However, an adaptive management approach (Option 3) provides an opportunity to assess the risk of population hybridisation by pairing animals of Pukerua Bay origin and comparing survival and fecundity of those offspring to that of hybrid offspring (about 30\% juvenile mortality, D Keall, pers. comm.) over several generations. If hybrid animals survive and reproduce as well as or better than pure offspring, animals from Middle Island could be intentionally introduced to the captive population to increase the founding population size. Hybrid animals could comprise a founder group for the proposed translocation to a predatorfree site. Whilst animals from Middle island could be released onto a predator-free island with captive animals (Option 4), there would be little opportunity to evaluate survival and fecundity. However, the non-genetic risks, including the potential disruption of social interactions and introduction of parasites or disease, and the potential value of preserving the historical integrity of a population (Schwartz 1994; Edmands 2007) need to be assessed by managers independently of genetic risks. As animals are extremely rare at Pukerua Bay, addition of skinks to the captive breeding programme from an island population would be beneficial from both genetic and demographic perspectives. 


\section{CHAPTER THREE}

\section{Genetic structure and individual fitness following translocation of a small lizard}

\subsection{Abstract}

Translocations of threatened species are often founded by a small number of individuals, but maximising genetic diversity is often a criterion for founder selection. Translocation of pregnant females has been proposed as a means of maximising productivity and genetic diversity, but it is unclear whether the release of pregnant females increases the effective number of founders. Ten male and 20 gravid female egg-laying skinks (Oligosoma suteri) were translocated from Green Island to Korapuki Island, New Zealand in 1992. We sampled the populations on both Green and Korapuki Islands to examine the effect of translocation on the genetic structure and fitness of egg-laying skinks in the 14 years following release. The population on Korapuki Island showed multiple genetic signatures of a bottleneck that were not detected in the population on Green Island. At the individual level, juveniles on Korapuki Island were more homozygous than adults on Korapuki and Green Islands. However, we did not find evidence of inbreeding depression using two performance-based surrogates of fitness. Further, the population on Korapuki Island had a significantly larger effective population size than would have been expected by translocation of 30 skinks, based on 10,000 simulated populations. The translocation of gravid females aided in increasing the effective number of founders, and may be a viable option for maximizing genetic diversity in translocated populations, particularly for longlived species. However, the continued loss of genetic variation in translocated populations may have more insidious long-term consequences, such as the loss of adaptive potential, which cannot be assessed in the short-term.

\subsection{Introduction}

Translocation is a common tool for species conservation and ecosystem restoration. The goal of translocation projects is to establish a viable population in an area from which the species has become extirpated (IUCN 1987). Translocations of threatened species generally have low success rates (Griffith et al. 1989; Dodd \& Seigel 1991; Germano \& 
Bishop 2009), but little is known about the causes of failure or the reasons for success (Armstrong \& McLean 1995). Although it is generally accepted that maximising genetic diversity in translocated populations is important (Fitzsimmons et al. 1997; Armstrong \& Seddon 2008), founder group sizes are often small, particularly for threatened and endangered species (Griffith et al. 1989).

A sudden reduction in population size (a bottleneck) can reduce genetic diversity and hence reduce evolutionary potential and increase the risk of extinction (Frankham 1999; 2005). Populations show several measurable genetic patterns after bottlenecks, including the loss of rare alleles and reduced allelic diversity (Allendorf 1986), a mode shift in allele frequency distribution (Luikart et al. 1998), non-random association of alleles at different loci (gametic disequilibrium, Hill 1981; Allendorf \& Luikart 2007), and an excess of heterozygosity relative to that expected in a non-bottlenecked population with the same number of alleles (Cornuet \& Luikart 1996; Luikart \& Cornuet 1998).

Inbreeding following genetic bottlenecks can cause additional losses of genetic diversity and an increase in the frequency of deleterious alleles. Inbreeding can have negative effects on the health and fitness of individuals and the stability of populations (Keller \& Waller 2002; O'Grady et al. 2006). The loss of genetic diversity is frequently correlated with a loss of individual fitness in wild populations (Crnokrak \& Roff 1999; Coltman \& Slate 2003). Inbred individuals often have a reduced probability of survival (Keller 1998; Coulson et al. 1999), lower reproductive output (Slate et al. 2000; Amos et al. 2001), or increased susceptibility to disease (Coltman et al. 1999; Cassinello et al. 2001). The influence of genetic bottlenecks on individual fitness also has consequences at population level (Newman \& Pilson 1997). For example, low hatching rates in birds are associated with small founder groups (Briskie \& Mackintosh 2004), and mean litter size relative to maternal body size was lower in an isolated population of adders (Vipera berus) than non-isolated populations (Madsen et al. 1996). Because of their effects at individual and population levels, inbreeding and reduced genetic diversity directly influence extinction risk in wild populations (Saccheri et al. 1998). It has been suggested that species with a history of small population size and frequent inbreeding (e.g. island endemics) may be less susceptible to the effects of inbreeding because they have been purged of deleterious alleles (Craig 1991). However, purging is unlikely to reduce inbreeding depression (Ballou 1997; Frankham 2001), and inbreeding depression has been detected in many island endemics (Jamieson et al. 2006). The loss of genetic 
diversity combined with inbreeding depression in a small reintroduced population could negatively affect the probability of population establishment.

Despite the known genetic consequences of small population size, translocations of threatened species are often founded by a small number of individuals to limit the impact on the source population (Griffith et al. 1989; Towns \& Ferreira 2001). Minimising the loss of genetic diversity is often considered important in translocation planning. Translocation of pregnant females has been used as a means of maximising productivity and genetic diversity (Towns \& Ferreira 2001; Nelson et al. 2002a), but it is unclear whether the release of pregnant females increases the effective number of founders. Stress during translocation may induce abortions and/or changes in sex ratio bias (e.g. rhinoceroses, Linklater 2007), or females may not be able to find suitable nest sites after release.

It is assumed that translocations cause genetic bottlenecks, but empirical evidence is still equivocal. Reduced heterozygosity and allelic diversity relative to the source population are well documented in translocated populations (Fitzsimmons et al. 1997; Williams et al. 2000; Swanson et al. 2006), but other bottleneck signatures such as an excess of heterozygosity relative to a population at equilibrium are less commonly described (but see Maudet et al. 2002). This bottleneck signature was associated with a translocated population of elk (Cervus elaphus) that remained smaller than 50 individuals for 50 years (due to legal harvest), but was not detected in a larger translocation that expanded rapidly (Williams et al. 2002), and no evidence of a genetic bottleneck was detectable when multiple source populations were used in a translocation of American martens (Martes americana, Swanson et al. 2006).

\subsubsection{Translocation of the egg-laying skink}

The introduction of mammalian predators during the human colonisation of $\mathrm{New}$ Zealand led to radical changes in abundance and distribution of native fauna (Towns \& Daugherty 1994). For example, at least $40 \%$ of the reptile and amphibian species are restricted mainly or entirely to offshore islands free of introduced mammals (Daugherty et al. 1994). Translocation of threatened species between islands has therefore become a primary conservation strategy in New Zealand (Armstrong \& McLean 1995; Saunders 1995). 
Egg-laying skinks (Oligosoma suteri) are shore-dwelling, oviparous reptiles (up to $108 \mathrm{~mm}$ snout-vent length (SVL), Hardy 1977) that live 20 years. These semi-aquatic lizards are restricted to boulder beaches and rocky shore platforms (Towns 1975a). Due to the effects of introduced predators, they have a fragmented distribution on islands and a few isolated mainland sites around northern New Zealand (Towns et al. 2003). In 1992, 30 adult egg-laying skinks were translocated from Green Island ( $3 \mathrm{ha}$ ) to Korapuki Island (18 ha). Both islands are found in the Mercury Island group ( $\left.36^{\circ} 40^{\prime} \mathrm{S}, 175^{\circ} 52^{\prime} \mathrm{E}\right)$, within $1.5 \mathrm{~km}$ of each other. Green Island has remained unmodified and predator-free, whereas Korapuki Island has a history of extensive modification by humans (Hicks et al. 1975) and introduced mammals (Towns 1991). Restoration of island ecosystems in New Zealand commonly involves eradication of all introduced mammals and the translocation of native species (Towns \& Ferreira 2001; Towns \& Broome 2003). Korapuki Island has been one such target of restoration efforts, including the eradication of rabbits (Oryctolagus cuniculus) and Pacific rats (Rattus exulans) and translocations of several species of invertebrates and reptiles, since 1986 (Towns 2002; Towns \& Atkinson 2004).

The translocation of egg-laying skinks to Korapuki Island was designed to minimise damage to the population on Green Island, but maximize productivity on Korapuki Island. Therefore, a small founder group of 10 males and 20 gravid females was released (Towns \& Ferreira 2001). Thus, the number of genetic founders could be 50 (if each female had mated with a different male) or more (if multiple paternity is common in O. suteri). The population on Korapuki Island was monitored at least twice annually until 2000 and at least biannually thereafter to obtain data on survival, recruitment, and population growth. Survivorship of founders in the first year was $68 \%$ for females and $72 \%$ for males, and population growth was detected within three years of release (Towns \& Ferreira 2001).

In this study, we examine the genetic structure and fitness of egg-laying skinks on Korapuki Island to determine whether the release of gravid females reduces the effects of genetic drift after translocation. We first test for several signatures of genetic bottlenecks, and ask how well genetic diversity was maintained during a period of population establishment by comparing genetic diversity in different cohorts. We then examine whether the release of gravid females had an influence on the effective number of founders by simulating genetic diversity in 10,000 translocated populations. Lastly, we examine whether the translocation of egg-laying skinks is associated with individual 
fitness consequences due to inbreeding using two performance-based surrogates of fitness.

\subsection{Methods}

\subsubsection{Animal collection and microsatellite genotyping}

We caught 185 egg-laying skinks on Korapuki Island and 53 on Green Island using pitfall traps baited with cat food. Trapping on Korapuki Island was from 14 November to 5 December 2006 (late Austral spring) and on Green Island from 1-4 March 2007 (Austral summer). Tail tissue $(\sim 3 \mathrm{~mm})$ from each trapped animal was removed using a sterile scalpel blade. Additionally, toe samples from 15 skinks on Korapuki Island were collected between 1996 and 1999. All tissue samples were stored in 70\% ethanol. Total genomic DNA extraction was performed using a standard proteinase $\mathrm{K}$ phenol-chloroform protocol (Sambrook et al. 1989) followed by ethanol precipitation. DNA was quantified using a NanoDrop ${ }^{\oplus}$ ND-1000 Spectrophotometer at $260 \mathrm{~nm}$.

All individuals were genotyped at 10 microsatellite loci (Oligr1, Oligr2, Oligr3, Oligr4, Oligr8, Oligr10, Oligr13, Oligr14, Oligr19, and Oligr20, Berry et al. 2003) adapted with 5' M-13 tags (Schuelke 2000). PCR was set up and carried out as outlined in Miller et al. (2009b, Chapter 2). PCR products were run on an ABI3730 Genetic Analyzer (Applied Biosystems, Inc.), and allele sizes were scored manually using GENEMAPPER 3.7 (Applied Biosystems, Inc.).

\subsubsection{Fitness measures}

Two measures of performance were used as surrogates of fitness of skinks on Korapuki Island: maximum sprint speed and dive duration. Sprint speed is linked to biological fitness of lizards because of key roles in foraging (Greenwald 1974), social interactions (Garland et al. 1990b), and predator escape (Christian \& Tracy 1981). Diving is a means of predator escape in many species of semi-aquatic lizards, including egg-laying skinks (Whitaker 1968; Miller 2007, Appendix 2), and may be a good measure of performance (Hare \& Miller in review). Standard morphometric measurements (mass, SVL, vent-tail length (VTL), tail regeneration length, and hind-limb length (HLL)) were taken. Sex of adults was determined by eversion of the hemipenes in males; juvenile sex could not be 
determined. Gravidity status and an estimate of clutch size of females were determined by abdominal palpation.

For full details on animal husbandry and performance tests, see Miller et al. (in review-a, Appendix 1). Briefly, three sprint tests were conducted on a plastic racetrack ( $80 \mathrm{~mm} \mathrm{x}$ $1.5 \mathrm{~m}$, modified from Huey et al. 1981). The maximum sprint speed over $0.25 \mathrm{~m}$ was used, as this is an ecologically relevant measure for egg-laying skinks, which inhabit rocky beaches where long sprints are not necessary to reach cover (Towns 1975a).

Three voluntary dive trials were conducted per animal in an artificial rock pool (see Hare \& Miller in review). All dive trials were conducted on the same day as, but after completion of, sprint trials. Some skinks did not dive, and instead swam about on the surface of the water. If an animal did not dive after $10 \mathrm{~s}$, we removed it from the experiment. We recorded the time to voluntary emergence (hereafter "dive duration") using a manual stopwatch (accurate to $1 \mathrm{~s}$ ).

\subsubsection{Data analysis}

Prior to analyses, we calculated the frequency of null alleles in FREENA (Chapuis \& Estoup 2007), and found that one locus (Oligr3) had a high frequency of null alleles in the Korapuki and Green Island populations ( 0.25 and 0.28 , respectively). Oligr3 was excluded from further analyses, because null alleles reduce evidence of intra-population genetic diversity (Paetkau \& Strobeck 1995).

Our aim was to make comparisons between both the source and translocated populations and within the translocated population over time. We therefore divided all samples into three independent groups for all analyses (except AMOVAs, see below): "Green Island" ( $n=85)$, "Korapuki adults" ( $n=126)$, and "Korapuki juveniles" ( $n=42$, Figure 3.1). The Green Island group was comprised of animals caught on Green Island $(n=53)$, founders of the Korapuki Island translocation recaptured in $2006(n=5)$, and effective founders of the Korapuki Island translocation (i.e. offspring of the 20 gravid female founders in the year of release, and thus matings on Green Island, $n=27$ ). Korapuki adults included only mature skinks ( $\geq 75 \mathrm{~mm} \mathrm{SVL}$ ) caught on Korapuki Island that were offspring of matings on Korapuki $(n=126)$. These animals represent the F1-F4 generations after translocation, based on a minimum age at maturity of three years 
(Towns 1975b). Korapuki juveniles included only immature skinks ( $<75 \mathrm{~mm} \mathrm{SVL)} \mathrm{on}$ Korapuki Island that represent the most recent cohort in the translocated population, and include individuals from the F1-F5 generations ( $n=42$, Figure 3.1).

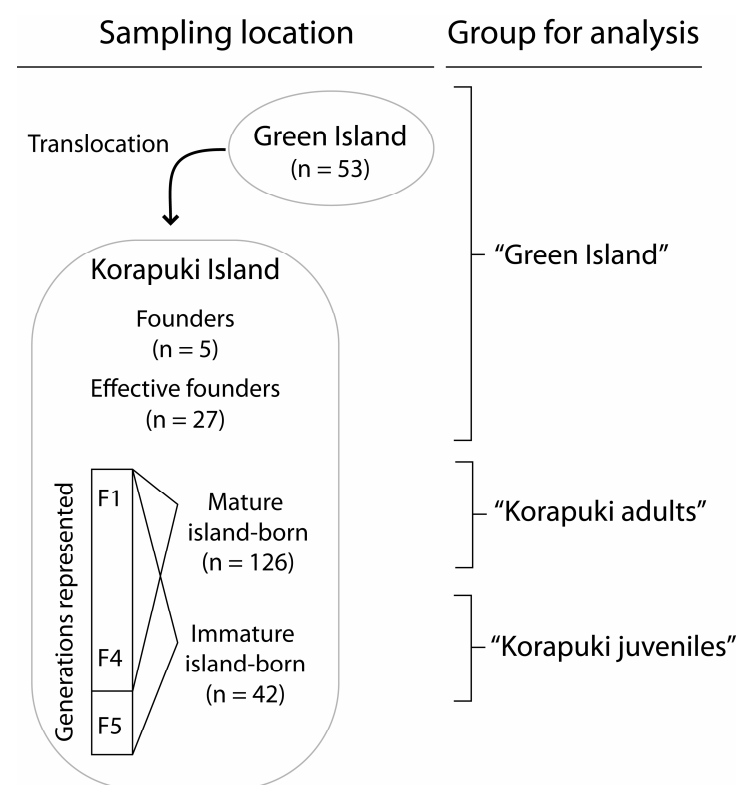

Figure 3.1 Sampling scheme showing locations where and numbers of (in brackets) egglaying skinks sampled, how groups were designated for bottleneck analyses, and the degree of generation overlap represented by skinks born on Korapuki Island. Ten male and 20 gravid female egg-laying skinks were translocated from Green Island to Korapuki Island in 1992. The 'effective founders' are offspring produced by the 20 gravid females in the year of translocation (i.e. offspring resulting from mating on Green Island).

\section{Genetic bottleneck effects}

Departures from Hardy-Weinberg proportions (Guo \& Thompson 1992) were calculated in GENEPOP 4.0 (Rousset 2008). Tests of significance were combined over all loci using Fisher's combined probability test. Significance was assumed at $p<0.05$. To test for differences in allele frequencies, we conducted pairwise analyses of molecular variance (AMOVA, Excoffier et al. 1992) in GENALEX 6.1 (Peakall \& Smouse 2006). When conducting AMOVAs, we removed founders and effective founders of Korapuki Island, as they are direct ancestors of the Korapuki adult and juvenile groups and would bias estimates of differentiation. Thus, it was a direct comparison of animals trapped on Green Island with the current population on Korapuki Island.

We tested for the presence of bottleneck effects in each group (Green Island, Korapuki adults, and Korapuki juveniles) using several measures. Using FSTAT 2.9 (Goudet 1995), we first calculated allelic richness (number of alleles corrected for sample size) of each 
population. Allele frequency mode shifts (Luikart et al. 1998) were assessed graphically. We tested for gametic disequilibrium within each population using a Fisher's exact test for all pairwise locus comparisons in GENEPOP 4.0 (Rousset 2008). Lastly, BOTTLENECK 1.2.02 (Cornuet \& Luikart 1996; Piry et al. 1999) was used to test for an excess of heterozygosity relative to a population at mutation-drift equilibrium. We used a statistically conservative two-phase model of mutation (Di Rienzo et al. 1994), which assumed 90\% of mutations are single-step and $10 \%$ are multi-step (with a variance of 10\%). Significance of heterozygosity excess over all loci was assessed with a Wilcoxon sign-rank test (Luikart \& Cornuet 1998). The statistic $M$ (the ratio of the number of alleles to the range in allele size, Garza \& Williamson 2001) could not be calculated because several loci in at least one population violated the test requirement of having empty allelic states within the range of common alleles (frequency $>0.1$ ).

Estimates of the effective population size $\left(\mathrm{N}_{\mathrm{e}}\right)$ of each group were generated using four methods. One-sample estimates were made using the linkage disequilibrium method (implemented in LDNe, Waples \& Do 2008) and an approximate Bayesian computation (using ONeSAMP, Tallmon et al. 2008). Using the linkage disequilibrium method, we excluded all alleles with frequencies less than 0.05 (Waples 2006) and used 95\% confidence intervals adjusted using the jackknife method. We generated $\mathrm{N}_{\mathrm{e}}$ estimates in ONeSAMP using a range of prior values (expected $N_{e}$ ranged from 2-500), but these changes did not affect the estimates. Two temporal estimates were made using Green Island as the first temporal sample (generation 0 ) and the Korapuki adult and juvenile groups as a second temporal sample (generation 2). The moments-based method described by Waples (1989) infers $\mathrm{N}_{\mathrm{e}}$ from the standardised difference in allele frequencies. The likelihood-based method described by Wang (2001) uses the rate of coalescence of alleles between sample periods to infer $\mathrm{N}_{\mathrm{e}}$.

\section{Simulations of genetic diversity after translocation}

We used the programme BoTTLESIM 2.1 (Kuo \& Janzen 2003) to model the expected loss of heterozygosity and allelic diversity following the translocation to Korapuki Island. BOTTLESIM allows the user to specify the starting number of alleles, their frequency, and changes in population size each year. We used Green Island samples as genotypic input data, and created annual population size estimates using population parameters outlined by Towns \& Ferreira (2001). These annual population size estimates closely matched estimates made using deterministic age-specific models for the first 7 years after 
translocation to Korapuki Island (Towns \& Ferreira 2001) and the estimated population size on Korapuki Island after 14 years ( 400 animals, KA Miller, unpubl. data). We specified several other parameters, including $75 \%$ generation overlap $(0 \%=$ discrete generations, $100 \%=$ complete overlap), random mating, maximum longevity of 20 years, and age of maturation at 3 years. We ran 10,000 iterations of a large starting population $(5,000$ animals, representing Green Island) reduced to 30 individuals (translocation to Korapuki Island) and allowed to recover for 14 years (the year at which we sampled animals on Korapuki Island). Each iteration represents a separate population. We obtained raw genotypic data from the last year of each iteration (i.e. genotypes for all animals in 10,000 simulated populations). To estimate $N_{e}$, we randomly sampled 126 genotypes (i.e. the sample size of the Korapuki adult population) in each simulated population and calculated $\mathrm{N}_{\mathrm{e}}$ in LDNe. We repeated this analysis using 50 founders (20 females and 30 males) to represent the number of genetic founders if each gravid female mated with a different male on Green Island.

\section{Inbreeding and heterozygosity-fitness correlations}

We calculated two heterozygosity-based metrics of individual inbreeding (hereafter "heterozygosity values") using the macro provided by Bill Amos (http://www.zoo.cam.ac.uk/zoostaff/amos): standardised expected heterozygosity (Coltman et al. 1999) and homozygosity by locus (HL, Aparicio et al. 2006), which tend to perform well when inferring inbreeding from multilocus heterozygosity data. Standardised expected heterozygosity is individual heterozygosity standardised by the mean expected heterozygosity in the population. $\mathrm{HL}$ weights each locus by its allelic variability, while considering the identity of each allele. We used Green Island as the reference population in calculating allele frequencies, because including related individuals on Korapuki Island would bias results.

We first confirmed that high heterozygosity values were due to the combined effect of all loci, rather than just one locus, by separately removing each locus and recalculating heterozygosity values at the remaining 5 loci. Heterozygosity values were not influenced by any one locus more than others, as values were highly correlated among all possible combinations of 5 loci (Pearson correlation coefficient, $r>0.78, p<0.001$ for all correlations). To assess changes in inbreeding, we then tested for differences in heterozygosity values among groups (Green Island, Korapuki adults, and Korapuki juveniles) using a one-way ANOVA. Post-hoc tests were conducted using Tukey's HSD. 
Fitness data were transformed as required to meet assumptions of normality. Body condition was calculated as the residuals from fitted data using a linear regression of $\log$ (mass) on log(SVL). Tail loss was defined as SVL/(SVL+VTL). As there were differences in maximum sprint speed and dive duration among groups (Miller et al. in review-a, Appendix 1), animals were divided into groups based on age, sex, and gravidity status for heterozygosity-fitness correlation analyses: males, gravid females, and juveniles. Nongravid adult females $(n=15)$ were excluded from analyses because of a small sample size.

We analysed whether inbreeding influenced maximum sprint speed and dive duration separately for each group, while taking morphological traits into account. We ran a series of standard least squares and reverse stepwise multiple regressions with the log of sprint speed (or log of dive duration) as the dependent variable. Covariates were heterozygosity value, SVL, body condition, and tail loss. Clutch size was included as a factor for gravid females, and HLL was included as a covariate for sprint speed.

\subsection{Results}

Seven of the 10 surveyed microsatellite loci were polymorphic (7-14 alleles each, Table 3.1). All populations were in Hardy-Weinberg proportions at all loci $(p>0.05)$. Differentiation among all populations was low but significant (overall $\Phi_{\mathrm{PT}}=0.010, p<$ 0.001). Korapuki adults and juveniles did not differ in allele frequencies, but both groups differed significantly in allele frequency from Green Island (Table 3.2).

\subsubsection{Genetic bottleneck effects}

A total of 62 alleles were found in the Green Island population, 58 were found in the Korapuki Island adult population, and 54 were found in the Korapuki Island juvenile population. Mean allelic richness was slightly lower in Korapuki adults (9.0) and juveniles (9.0) than in the Green Island population (9.9, Table 3.1). However, at one locus (Oligr10), an allele was detected in Korapuki adults and juveniles, but not in Green Island animals. At a second locus (Oligr8), an allele was found in Korapuki juveniles and the Green Island population, but not in Korapuki adults. Significant gametic disequilibrium $(p<0.05)$ was detected in seven of 15 pairwise locus comparisons in the Korapuki Island adult population after sequential Bonferroni correction. No significant gametic disequilibrium was detected in either the Green Island or Korapuki Island juvenile populations. 


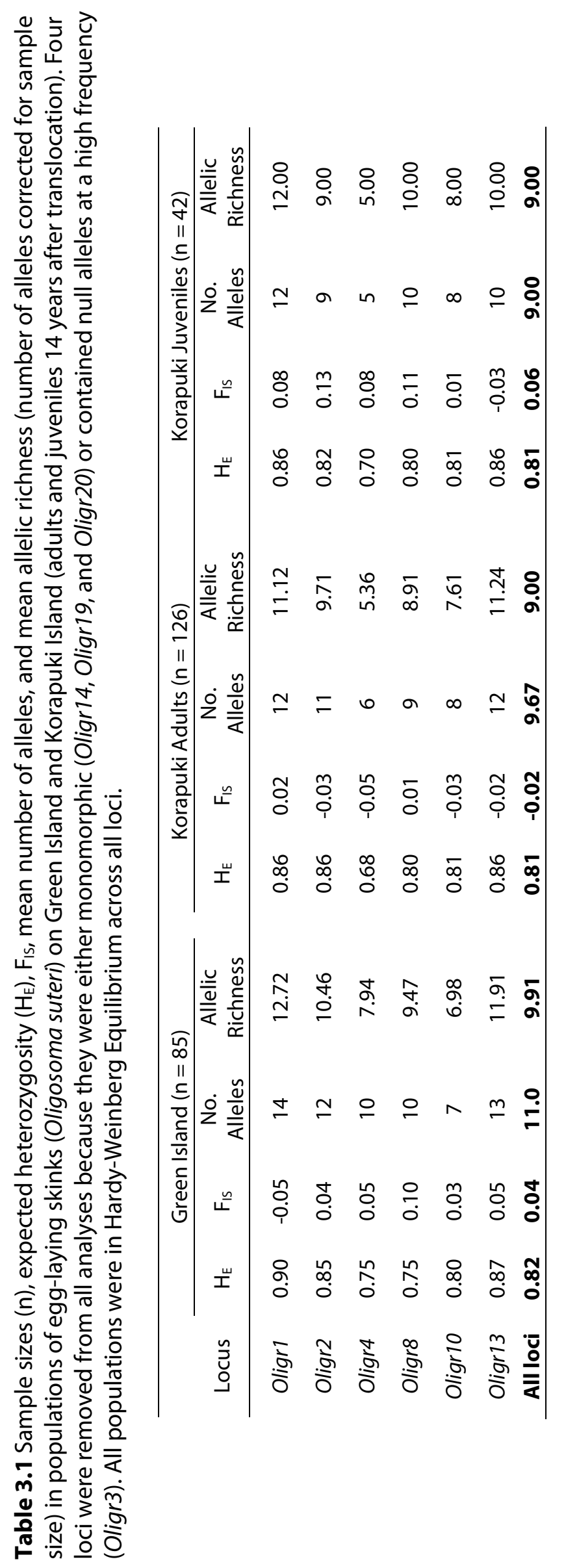


Table 3.2 Pairwise population $\Phi_{\text {PT }}$ estimates (below diagonal) and $p$-values (above diagonal) for each sampling group. Green Island was the source population and adults (F1-F4) and juveniles (F1-F5) were sampled in the translocated population (Korapuki Island).

\begin{tabular}{lccc}
\hline & $\begin{array}{c}\text { Green } \\
\text { Island }\end{array}$ & $\begin{array}{c}\text { Korapuki } \\
\text { Adults }\end{array}$ & $\begin{array}{c}\text { Korapuki } \\
\text { Juveniles }\end{array}$ \\
\hline Green Island & -- & 0.001 & 0.026 \\
Korapuki Adults & 0.021 & -- & 0.430 \\
Korapuki Juveniles & 0.014 & 0.000 & -- \\
\hline
\end{tabular}

There was no mode shift of allele frequency distributions in any group surveyed

(Figure 3.2), but the mean proportion of rare alleles was lower in Korapuki juveniles (0.44), and Korapuki adults (0.57) than on Green Island (0.67), despite our sample size being largest in Korapuki adults. Similarly, Korapuki Island adult and juvenile populations had a significant excess of heterozygosity relative to that expected at mutation-drift equilibrium ( $p=0.008$ and 0.02 , respectively). The Green Island population did not show an excess of heterozygosity $(p=0.58)$.

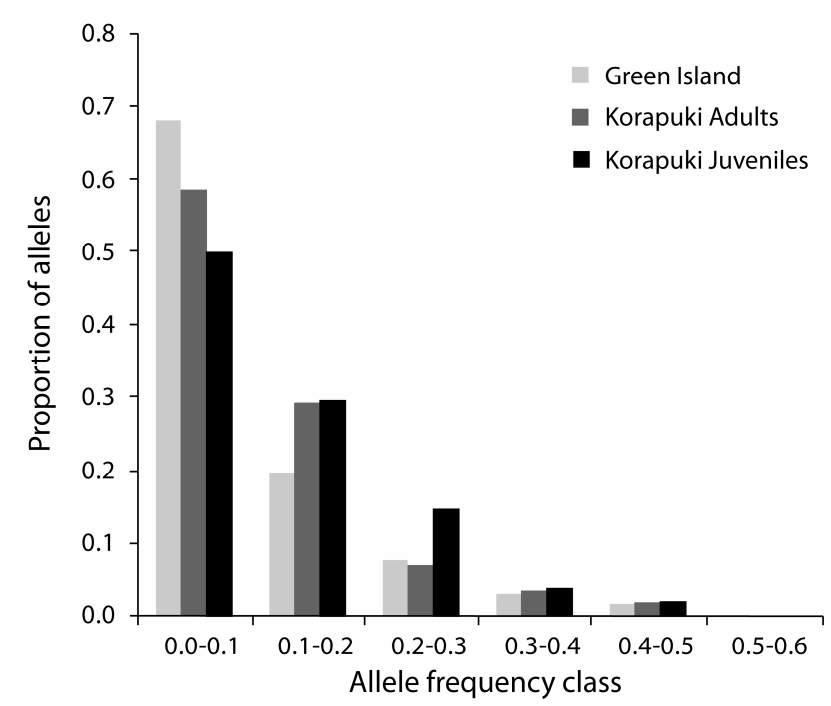

Figure 3.2 Allele frequency distributions in Green Island animals (source population), Korapuki adults, and Korapuki juveniles. No population showed a significant mode shift, but rare alleles were less common in the translocated population (Korapuki adults and juveniles).

\subsubsection{Estimates of effective population size}

Estimates of $\mathrm{N}_{\mathrm{e}}$ were similar using all methods (Table 3.3) except the moments-based method, which often generates estimates that are biased high (Luikart et al. 1999; Wang 2001). Both temporal estimates had wider confidence intervals than the one-sample 


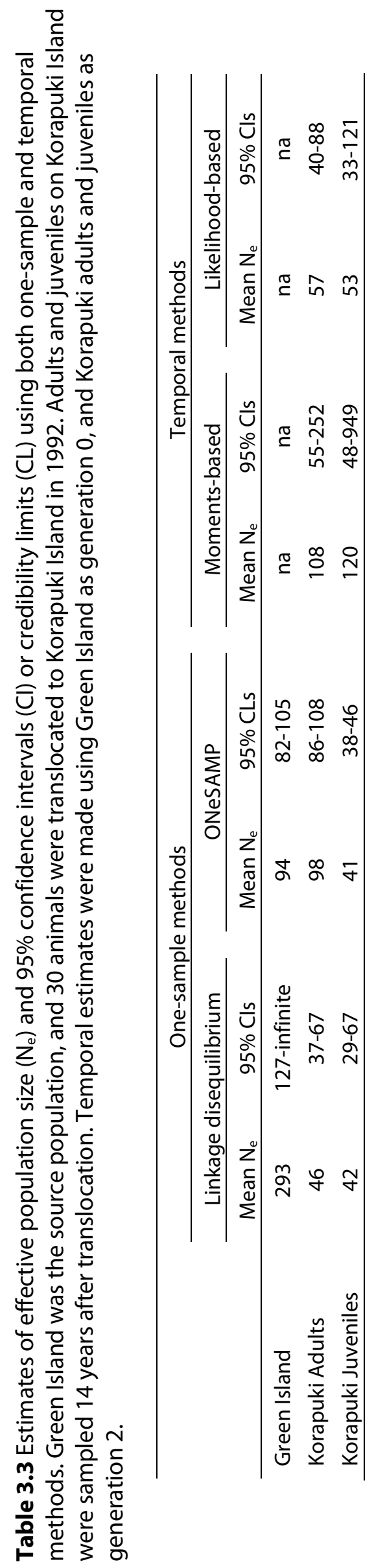


estimates. Both one-sample estimates indicate a reduction in $\mathrm{N}_{\mathrm{e}}$ consistent with translocation history, and the $\mathrm{N}_{\mathrm{e}}$ in the Green Island population (293) was much larger than the Korapuki Island populations. However, estimates of $\mathrm{N}_{\mathrm{e}}$ in the Korapuki adult and juvenile populations were higher than 30 (the size of the founder group). The estimate of $\mathrm{N}_{\mathrm{e}}$ from Bayesian computation for Korapuki Island adults was similar to Green Island (Table 3.3), but our data violates the test assumption that the population is in mutationdrift equilibrium.

The actual $H_{E}$ and allelic diversity for Korapuki adults after 14 years ( 0.81 and 9.67, Table 3.1) were more similar to the values predicted by 10,000 simulations of a population with 50 genetic founders (simulated mean $\mathrm{H}_{\mathrm{E}}=0.80 \pm 0.03$ and number of alleles $=9.42 \pm 0.86$ ) than values predicted by 30 founders (simulated mean $\mathrm{H}_{\mathrm{E}}=0.79 \pm 0.03$ and number of alleles $=9.03 \pm 0.82$ ). In the simulated populations, the greatest loss of allelic diversity took place during translocation, but allelic diversity declined steadily over 14 years (Figure $3.3 a, b)$. The mean $\mathrm{N}_{\mathrm{e}}$ for simulated populations of 30 founders $(31 \pm 0.06)$ did not fall within the $95 \% \mathrm{Cls}$ of the $\mathrm{N}_{\mathrm{e}}$ estimate for Korapuki adults (37-67). The mean $\mathrm{N}_{\mathrm{e}}$ for simulated populations of 50 founders ( $37 \pm 0.06$ ) was smaller than, but not significantly different to the $\mathrm{N}_{\mathrm{e}}$ estimate for Korapuki adults.

\subsubsection{Inbreeding and heterozygosity-fitness correlations}

Heterozygosity values were highly correlated $(r=-0.988, p<0.001)$, and gave similar results in all analyses. We therefore present only results using $\mathrm{HL}$ (homozygosity by locus). Higher $\mathrm{HL}$ values indicate that individuals are more homozygous. Mean $\mathrm{H}_{\mathrm{E}}$ was not different among any group (Table 3.1), but mean individual $\mathrm{HL}$ differed among groups $(p=0.024$, Figure 3.4). Juveniles had higher mean individual $\mathrm{HL}(0.24 \pm 0.03$, range $=$ $0.00-0.68)$ than both adults on Korapuki Island $(0.17 \pm 0.02$, range $=0.00-0.51, p=0.027$ ) and animals on Green Island $(0.17 \pm 0.01$, range $=0.00-0.52, p=0.037)$, which most likely indicates that only the juvenile group includes individuals with high pedigree inbreeding values. Mean individual HL of adults on Korapuki Island did not differ from animals on Green Island $(p=0.997)$.

We measured the maximum sprint speed of 63 males, 43 gravid females, and 30 juveniles on Korapuki Island. Maximum sprint speed was not explained by $\mathrm{HL}$ in either males or gravid females. HL was the only factor that explained juvenile sprint speed, and 


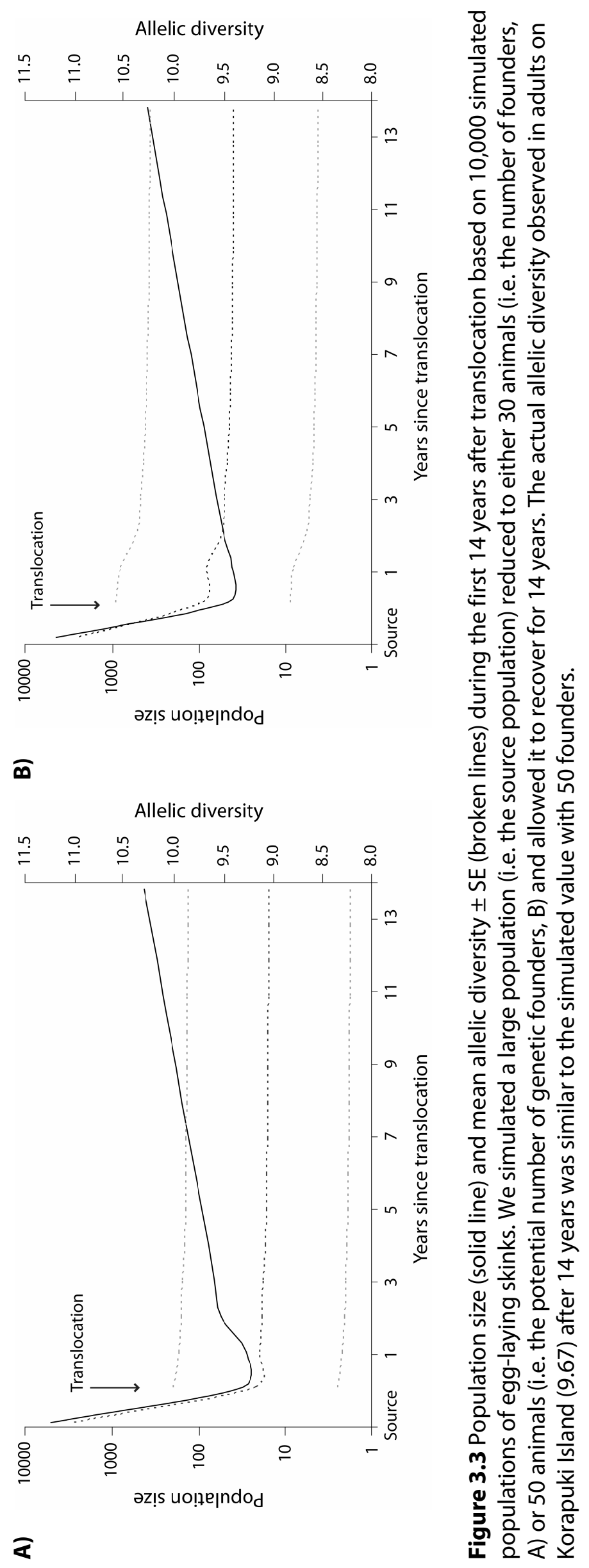


accounted for $18 \%$ of the variation in sprint speed. However, juveniles with higher HL ran faster than juveniles with lower $\mathrm{HL}$ values $\left(F_{1,28}=6.18, p=0.019\right)$. We measured maximum dive duration of 63 males, 39 gravid females, and 31 juveniles, and maximum dive duration was not explained by $\mathrm{HL}$ in any group. The relationships between the morphological measurements and performance variables are discussed in Miller et al. (in review-a, Appendix 1).

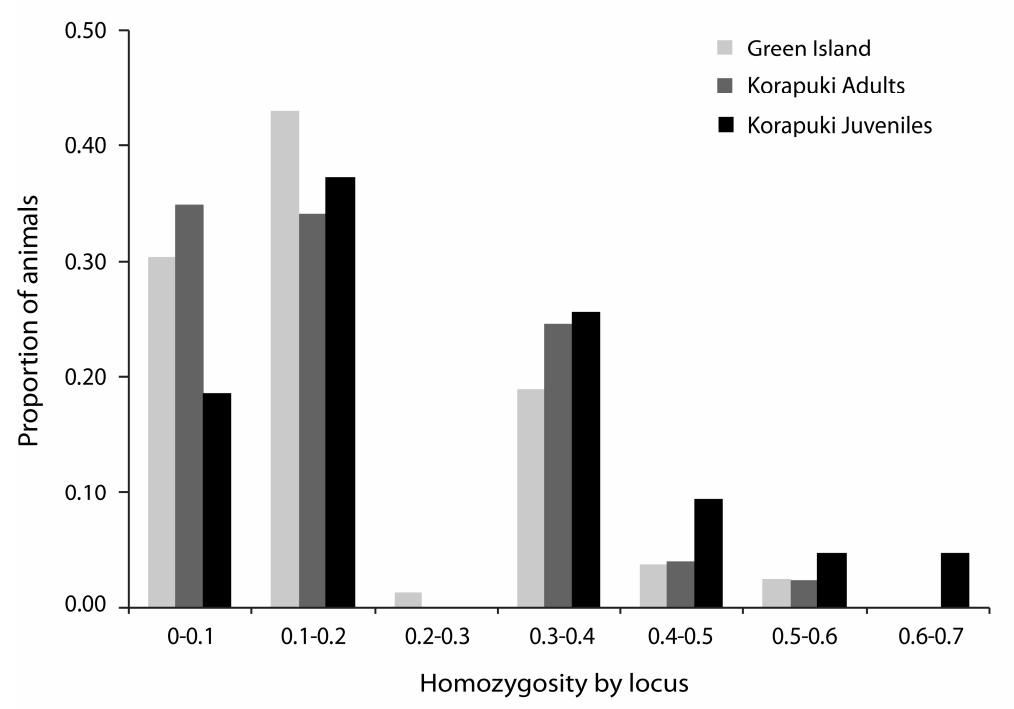

Figure 3.4 Distribution of individual homozygosity by locus $(\mathrm{HL})$ values in Green Island animals (source population), Korapuki Island adults, and Korapuki Island juveniles. Higher $\mathrm{HL}$ values indicate that individuals are more homozygous. Mean individual $\mathrm{HL}$ was not different for Korapuki Island adults and Green Island, but Korapuki Island juveniles had a higher mean individual $\mathrm{HL}$ than both groups.

\subsection{Discussion}

Egg-laying skinks translocated from Green Island to Korapuki Island show several expected signatures of a genetic bottleneck after translocation, but retain high levels of genetic diversity. Skinks on Korapuki Island have high heterozygosity and retain $\sim 80 \%$ of the allelic diversity of Green Island 14 years after translocation, but the proportion of rare alleles has decreased over time. Gametic disequilibrium, an excess of heterozygosity (relative to expected heterozygosity at equilibrium), and reduced effective population size on Korapuki Island relative to Green Island were detectable in a short time-frame after translocation, and reflect their translocation history (i.e. a bottleneck). Juveniles in the translocated population are more homozygous than adults, despite these groups representing overlapping generations, but adults on Korapuki Island were not more homozygous than animals on Green Island. 


\subsubsection{Genetic bottleneck effects}

Tests for genetic bottleneck effects are often used to reveal historic or previously unknown reductions in population size. By examining the signatures of a genetic bottleneck in reintroduced populations with a well-documented reduction in population size, it is possible to validate their effectiveness in detecting genetic bottlenecks. Genetic drift after a population bottleneck may simultaneously cause a loss of alleles within populations and changes in allele frequencies (e.g. Ramstad et al. 2004; Stephen et al. 2005). Allelic richness was higher in the population on Green Island than in either the adult or juvenile populations on Korapuki Island. Rare alleles are most likely to be lost during bottlenecks (Allendorf 1986; Luikart et al. 1998) and were least frequent in the youngest cohort in the translocated population of egg-laying skinks (Korapuki juveniles). Allele frequencies in adults and juveniles on Korapuki Island were not significantly different, but both were significantly different from the population on Green Island. Pairwise $\Phi_{\text {PT }}$ values were positively correlated with the difference in allelic richness among populations. Thus, the differentiation between Green Island (the source) and Korapuki Island (the translocation) is most likely due to the loss of alleles during the translocation.

Recent population bottlenecks have several effects on genetic structure that persist over different time-scales. Bottlenecks in a previous generation could be responsible for significant gametic disequilibrium at unlinked loci even when the population size is large, but disequilibrium should decay quickly (Hedrick 2000). Although disequilibrium is rarely demonstrated empirically in recently bottlenecked populations (but see Miller et al. 2008), we found significant gametic disequilibrium in the adults on Korapuki Island (older cohorts), but not in juveniles (the youngest cohort) that represent later generations. Additionally, gametic disequilibrium was not detected in the population on Green Island. This observed pattern of gametic disequilibrium suggests that the bottleneck during translocation is responsible for the non-random association of alleles at different loci in adults on Korapuki Island. As the translocated population rapidly expanded, it is possible that disequilibrium decayed quickly, so that it was not observed in the juvenile cohort. However, it is also possible that we were not able to detect gametic disequilibrium in the juvenile cohort because of small sample size. Estimates of $\mathrm{N}_{\mathrm{e}}$ were similar in the adult and 
juvenile populations using the linkage-disequilibrium method, suggesting that the underlying gametic disequilibrium is equivalent in both groups.

Although gametic disequilibrium is a relatively transient signature of a bottleneck, an excess of heterozygosity (relative to a population in mutation-drift equilibrium) should be detectable for up to $4 \mathrm{~N}_{\mathrm{e}}$ generations (Cornuet \& Luikart 1996). Many studies examine the loss of heterozygosity and allelic diversity after translocation (Fitzsimmons et al. 1997; Williams et al. 2000; Stephen et al. 2005; Boessenkool et al. 2007), but relatively few have tested for an excess of heterozygosity in both the source and translocated populations (but see Maudet et al. 2002; Swanson et al. 2006). All translocated populations of Alpine ibex (Capra ibex) showed an excess of heterozygosity, but the source population showed the same bottleneck signature (Maudet et al. 2002). Conversely, there was an excess of heterozygosity in the adult and juvenile populations on Korapuki Island, but not in the source population (Green Island). This pattern suggests that the bottleneck caused by translocation, rather than a bottleneck on Green Island prior to translocation, produced the excess of heterozygosity.

\subsubsection{Maximising $N_{e}$ during translocation}

The reduction of genetic diversity caused by translocation will reduce evolutionary potential and increase the extinction risk (Newman \& Pilson 1997; Frankham 1999). Thus, minimising the reduction in $\mathrm{N}_{\mathrm{e}}$ during translocation may help avert long-term consequences (Reed et al. 2003). Pregnant females have often been translocated with the goal of maximising genetic diversity, but it has been unclear whether translocation of pregnant females has actually increased the effective number of founders. Egg-laying skinks on Korapuki Island have a significantly larger $\mathrm{N}_{\mathrm{e}}(46)$ than would be expected by translocation of 30 animals, based on 10,000 simulated populations, but had a 3- to 7-fold smaller effective population size than Green Island. The release of gravid females accounts for the larger $\mathrm{N}_{e}$, as these offspring represented additional diversity found in the source population, and the $\mathrm{N}_{\mathrm{e}}$ estimate is similar to that of a population with the same growth rate founded with 50 animals. In species where each founder requires a large resource investment (of either money or time), translocation of pregnant females presents a means of increasing the effective number of founders and reducing, but not eliminating, the impact of genetic drift immediately following translocation. 
When source populations are small, or when founders of a translocation are taken from captivity, pregnant females are valuable to both the source and the translocated populations, and release of pregnant females may come at a cost to the source population. In such cases, the trade-offs must be assessed relative to the specific goals of the programme (Earnhardt 1999). Captive-reared clutches are also often used for translocation for conservation reasons (Snyder et al. 1996), but the release of siblings may reduce the effective number of founders in these populations. Whilst it is important to include relatedness as a criterion for founder selection (e.g. Nelson et al. 2002a), studies are needed to assess how the release of only siblings affects genetic diversity and effective population size in translocated populations (Moore et al. 2008).

\subsubsection{Fitness consequences}

Individual heterozygosity value, determined from microsatellite loci, had a significant influence on maximal performance of juveniles (measured by sprint speed), but not in adults on Korapuki Island. Higher speeds generally result in greater survival and fitness in lizards and snakes (Jayne \& Bennett 1990; Husak et al. 2006; Irschick et al. 2008). Contrary to expectations, more homozygous juveniles ran faster. Therefore, this relationship does not provide evidence of inbreeding depression in egg-laying skinks on Korapuki Island, but our conclusions are limited by the fact that we did not measure the level of inbreeding directly. In using microsatellite-based metrics, it is assumed that heterozygosity in a small set of neutral markers is informative about the level of inbreeding from pedigrees, yet this assumption has been challenged (Balloux et al. 2004; Slate et al. 2004). Heterozygosity-based measures may reflect inbreeding when variation in the level of individual inbreeding is high and populations have genetically similar and dissimilar individuals, but the power to detect associations between heterozygosity and fitness is low when fewer than 10 loci are used (Slate \& Pemberton 2002).

If heterozygosity-based measures of inbreeding do accurately reflect true inbreeding in O. suteri, two alternate explanations could account for the positive correlation between sprint speed and inbreeding: random chance or directional selection. Correlations between heterozygosity and fitness are frequently detected in wild populations (e.g. Slate et al. 2000; Keller \& Waller 2002; Coltman \& Slate 2003). Although sprint speed is the most commonly used measure of performance in lizards (Van Damme \& Vanhooydonck 2001), it is an indirect surrogate of fitness and may not accurately predict survival in egg-laying 
skinks. There are circumstances in which speed may not reflect either the probability of survival or fitness, or may be maladaptive. In whiptail lizards (Aspidoscelis tesselata), selection only favours faster sprint speed in animals found in open habitats with sparse refuge where they are highly vulnerable to predation (Punzo 2007). Frequent stops and starts reduce overall speed of hatchling phrynosomatid lizards (Sceloporus spp.), but may be more effective for the avoidance of predators (see Brodie 1992; Andrews et al. 2000). Juvenile egg-laying skinks may easily find refuge in their boulder beach habitat, and individual egg-laying skinks may employ multiple escape tactics to avoid predation (Miller et al. in review-a, Appendix 1). Thus, the selective pressure on higher speed may be reduced, and in this situation, the observed relationship may be due to chance.

Alternately, directional selection for faster speed could result in inbreeding. Sprint speed is heritable (Tsuji et al. 1989; Garland et al. 1990a), and sexual selection favours faster males, who sire more offspring than slower males (Husak et al. 2006). In very small populations, fast animals could be inbred after a few generations under these conditions. This type of directional selection could have a strong influence on the maintenance of genetic diversity over time (see Chapter 5), but this force is seldom considered in translocated populations. Our data do not distinguish between the alternate explanations that the relationship between heterozygosity and sprint speed in juveniles is due to selection (i.e. a normal pattern in natural populations of egg-laying skinks), or that the relationship is due to chance. Discriminating between these two hypotheses would provide valuable information on the dynamics of inbreeding in this translocated population and the behaviour of egg-laying skinks.

It is possible that homozygous juveniles, despite having higher sprint speeds, are less likely to survive into adulthood. Juveniles on Korapuki Island were more homozygous than adults, despite strong overlap of generations in the two groups. Further, the range of individual heterozygosity values is larger in juveniles than adults. Juveniles would be more homozygous on average than adults when inbreeding is increasing (in generations following a bottleneck) or if homozygous juveniles do not survive into adulthood. In the former scenario, we would predict that adults on Korapuki Island would have a higher mean heterozygosity value than the source population. However, the mean heterozygosity value of adults on Korapuki Island was equivalent to animals on Green Island. Increased inbreeding and the continued loss of genetic variation in translocated 
populations may have more insidious long-term consequences, such as the loss of adaptive potential, that cannot be measured in a short time frame.

The population of egg-laying skinks on Korapuki Island shows several signatures of a bottleneck, but we found no clear evidence of inbreeding depression in the short-term. Inbreeding depression has been manifested in decreased survival (e.g. juvenile red deer, Coulson et al. 1999) and breeding success (e.g. song sparrows, Keller 1998), or increased disease susceptibility (e.g. Soay sheep, Coltman et al. 1999). We used more indirect surrogates of fitness, as it is often difficult to measure survival and breeding success in highly cryptic species. Thus, it may be harder to asses these direct fitness consequences of inbreeding in many species, particularly after translocation. However, inbreeding depression has been detected in other translocated populations. For example, highly inbred juvenile North Island robins (Petroica longipes) in a translocated population had a low probability of survival (Jamieson et al. 2007). The effects of inbreeding depression were short-lived, as close inbreeding became less frequent with increasing population size (Jamieson et al. 2007), and inbreeding dynamics in translocated populations still remain unclear.

\subsubsection{Conclusions}

When population size increases rapidly after translocation, the effects of inbreeding depression may deteriorate as highly inbred individuals become rarer (Jamieson et al. 2007), but signatures of the genetic bottleneck will be more enduring (this study). Recently translocated populations, particularly those translocated for conservation reasons, have a low probability of establishment in the short-term because of multiple non-genetic factors (Griffith et al. 1989; Dodd \& Seigel 1991), and these factors have large influences on the design of a translocation. Simultaneously, maximising genetic diversity in translocated populations may be essential for their long-term persistence.

Translocation of pregnant or gravid females may be a viable option for increasing the effective number of founders, and long-lived species may benefit from translocation of pregnant females. Further data are required from species with varied life history traits to determine how genetic bottlenecks during translocation influence inbreeding dynamics and the maintenance of genetic diversity over time. 


\section{CHAPTER FOUR}

\section{The effects of supplementation and serial translocation on genetic diversity in species with varied life histories}

\subsection{Abstract}

Maintaining genetic variation is important for the success of translocated populations, yet the founding event and genetic drift in the period of population growth may cause losses of genetic diversity after translocation. Here we test how life history traits influence the maintenance of genetic diversity after translocation, and model how management actions may further impact genetic diversity. Three species of skink (Reptilia: Scincidae) with different life histories were translocated to Korapuki Island, New Zealand between 1988 and 1992. We sampled source and translocated populations of each species to assess the genetic consequences of translocation, and modelled genetic diversity over 10 generations to evaluate the implications of plausible management techniques. In the 1418 years following translocation, the species with the lowest reproductive output (Cyclodina whitakeri) retained less heterozygosity than predicted by models, but the species with the highest output (Oligosoma suteri) retained more than predicted. Models showed that supplementation of the translocated populations with 30 additional animals would not reduce the loss of heterozygosity over 10 generations in $O$. suteri, but would for the species with low reproductive output (C. whitakeri and C. alani) by decreasing the time required to reach carrying capacity. Further, harvest of $30 \mathrm{C}$. whitakeri from Korapuki Island for a second-order translocation would accelerate the loss of heterozygosity after 10 generations in both the first- and second-order translocations. Supplementation of translocated populations will have the greatest effect during the initial period of population growth, as new animals will aid in rapid recovery from a bottleneck and limit the impact of genetic drift. Actions that slow population growth (e.g. harvesting from a relatively small population) should be avoided.

\subsection{Introduction}

Population bottlenecks reduce genetic diversity, and may result in short-term decreases in fitness (inbreeding depression, Jamieson et al. 2007), increased extinction risk, and 
reduced evolutionary potential (Frankham 1999; 2005). Indeed, extinction risk increases significantly with decreasing genetic diversity (Saccheri et al. 1998). Maintaining genetic diversity is an important goal for species management, yet management actions may impose bottlenecks. Translocation is a common tool for the conservation of threatened species, but translocated populations often have lower genetic diversity than their sources at neutral and functional loci (Williams et al. 2000; Miller \& Lambert 2004). Understanding and predicting how management actions affect genetic diversity in translocated populations is identified as a priority both for species recovery (e.g. Towns 1999) and the broader field of reintroduction biology (Armstrong \& Seddon 2008).

Translocations of threatened and endangered species generally have low success rates (Griffith et al. 1989), and raise a suite of issues surrounding the species' physiology, ecology, behaviour, and population dynamics that influence the likelihood of success (Dodd \& Seigel 1991; Armstrong \& Seddon 2008). Yet under even the best of circumstances, life history characteristics influence whether populations expand rapidly, and thus how well genetic diversity is maintained. Long-lived species with overlapping generations may retain a high proportion of genetic diversity over several generations when carrying capacity is large (Taylor \& Jamieson 2008), but smaller populations may suffer from inbreeding depression. Supplementation could be used to help restore such populations (e.g. Madsen et al. 1999), and has been suggested as a way to maintain genetic diversity in translocated populations with very small carrying capacity ( 100 individuals, Grueber \& Jamieson 2008). However, it is unclear how supplementation influences the maintenance of genetic diversity in translocated populations with larger carrying capacity (> 1,000 individuals).

Serial translocations are occasionally conducted with threatened and endangered species because source populations may be few and/or fragile. However, the effects of serial population bottlenecks are not well understood. Serial translocation has been shown to reduce genetic variation in some cases (Lambert et al. 2005), but not others (Taylor \& Jamieson 2008). In fact, genetic diversity did not differ between first, second, or even third-order translocations of South Island saddlebacks (Philesturnus carunculatus carunculatus, Taylor \& Jamieson 2008).

Here, we investigate the effect of translocation on genetic diversity in three species of skink with different life history traits. We also model how several potential management 
options may impact the maintenance of genetic diversity over 10 generations.

Specifically, we ask how well models predict the level of heterozygosity maintained over the period since release, how serial translocation would affect the maintenance of diversity, and whether supplementation (with 10 or 30 animals) would benefit translocated populations with large carrying capacities.

\subsubsection{Translocation of skinks to Korapuki Island}

Three species of skink were translocated to Korapuki Island, New Zealand between 1988 and 1992: Cyclodina alani (robust skink), C. whitakeri (Whitaker's skink), and Oligosoma suteri (egg-laying skink). These populations were monitored closely to determine postrelease survival and population growth. A fourth species was also translocated (C. oliveri, marbled skink), but was only monitored until breeding was detected (Towns \& Ferreira 2001). All of these species are nocturnal, ground-dwelling, and susceptible to predation by introduced mammals (Towns \& Daugherty 1994). Cyclodina alani and C. whitakeri are primarily forest-dwelling viviparous skinks that occupy seabird burrows, rocky areas, and forest litter (Robb 1986). Oligosoma suteri is a coastal oviparous species found on boulder beaches and rocky shore platforms (Towns 1975a). All three species are found mainly or entirely on predator-free offshore islands; C. alani and C. whitakeri are listed as threatened by the IUCN, with six and three natural populations, respectively (totalling $<35$ and $<20$ ha).

Translocations were all within the Mercury Island group, off north-eastern New Zealand (Figure 4.1), from Green Island (3 ha) and Middle Island (13 ha) that are naturally free of mammals, to Korapuki Island (18 ha), from which all introduced mammals (rabbits and Pacific rats) were eradicated by 1987 (Towns 1991). The translocations were planned to meet objectives for species recovery and ecosystem restoration (Towns et al. 1990; Towns \& Atkinson 2004), but were constrained by the need to minimise detrimental impacts on the parent populations and habitat on the source islands. Founder groups were small because the source islands are small and have a high density of seabird burrows, which create fragile soils and potential for extensive damage to the habitat. Each translocation was designed to have 30 founders, but actual founder group sizes were 14 for $C$. alani and 28 for $C$. whitakeri, because of post facto decisions to minimise damage to the parent populations (Table 4.1, Towns \& Ferreira 2001), and an inadequate number of captured animals indicated small source populations. 


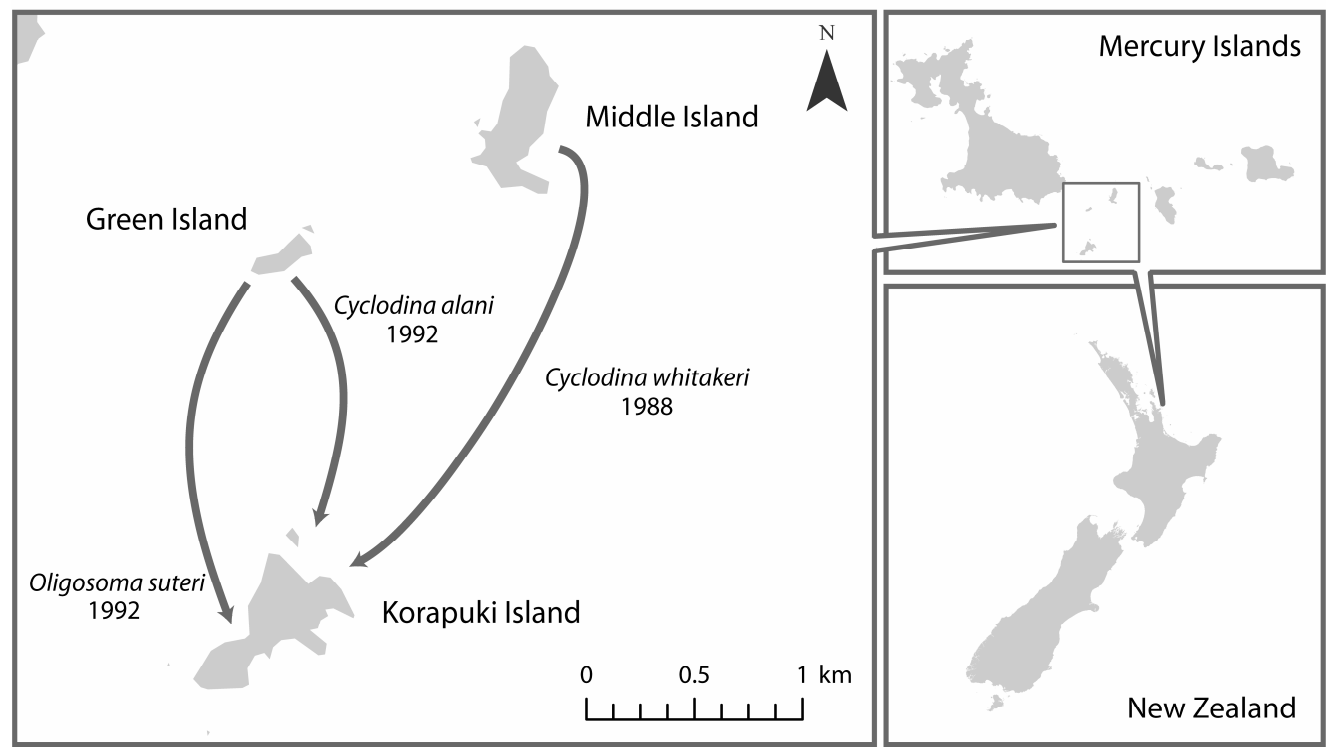

Figure 4.1 Map of the Mercury Islands, New Zealand, showing the source and translocation sites for Cyclodina alani, C. whitakeri, and Oligosoma suteri.

Survivorship in the 12 months following release on Korapuki Island was as low as $60 \%$ (C. whitakeri, Towns \& Ferreira 2001); sex ratios of the surviving founders were female-biased for each species, although the $O$. suteri release group was intentionally female-biased (1M:2F, Table 4.1). Population growth was measured for two of the three species by 1999 (Towns \& Ferreira 2001), and for all three subsequently (KA Miller \& DR Towns, unpubl. data). In order to protect the fragile ecosystems on Middle and Green Islands, Korapuki Island is the proposed source of future translocations of each species to other islands. These second-order translocations may be carried out as early as 2015, but the implications of serial translocation are unknown.

\subsection{Methods}

\subsubsection{Sample collection and microsatellite genotyping}

We attempted to catch animals in both the source and translocated populations using baited pitfall traps and by searching at night. However, we did not catch any C. alani on the source island (Green Island) over 180 trap nights and 20 hours of searching. We therefore caught $C$. alani on Middle Island to provide estimates of genetic diversity in a natural, non-bottlenecked population, but we could not make direct comparisons between the source and translocated populations. Populations of $O$. suteri on Middle and Green Islands have similar levels of heterozygosity (KA Miller, unpubl. data), and historic levels of genetic diversity in C. alani on the two islands may have been equivalent. 
Table 4.1 Life history characteristics, release dates, founder group sizes, and estimated carrying capacity for the skink species translocated to Korapuki Island.

\begin{tabular}{lccc}
\hline & Cyclodina alani & Cyclodina whitakeri & Oligosoma suteri \\
\hline Longevityy $^{1,2}$ & 35 & 20 & 20 \\
Age at maturity $^{2,3,4}$ & 5 & 4 & 3 \\
Mean annual clutch size $^{2,3,4,5}$ & 3 & 1 & 3.7 \\
Generation interval $^{6}$ & 15 & 10 & 10 \\
& & & \\
Korapuki Island translocation & 1992 & 1988 & 1992 \\
Year of release $^{2}$ & $5: 7: 2$ & $8: 5: 15$ & $10: 20$ \\
Sex ratio at release $^{2,3,7}$ & $2: 8$ & $6: 10: 1$ & $7: 15$ \\
Sex ratio of recaptured founders $^{2,3,7}$ & 4100 & 2600 & 5000 \\
Estimated carrying capacity $^{5,8}$ & & & \\
\hline
\end{tabular}

\footnotetext{
${ }^{1}$ Estimated from annual survival of founders after translocation (this study)

${ }^{2}$ Towns \& Ferreira 2001

${ }^{3}$ Towns 1994

${ }^{4}$ Towns $1975 b$

${ }^{5}$ Southey 1985

${ }^{6}$ Estimated from data on longevity and age at maturity (this study)

${ }^{7}$ (Male:Female:Unknown)

${ }^{8}$ Whitaker 1973
}

We removed $\sim 3 \mathrm{~mm}$ of tail tissue from each animal using a sterile scalpel blade. Skinks on Korapuki Island were sampled between November 2006 and March 2007 (all species) and at release (C. whitakeri only). Additionally, we collected toe tissue samples from $O$. suteri on Korapuki between 1996 and 1999; these samples represent offspring of females that were gravid at release (i.e. animals from matings on Green Island) and are thus effectively founders of the Korapuki population (Miller et al. in review-b, Chapter 3). We sampled source populations between March 2007 and March 2008. All samples were stored in 70\% ethanol.

We extracted total genomic DNA using a standard proteinase $\mathrm{K}$ phenol-chloroform protocol (Sambrook et al. 1989) followed by ethanol precipitation, and quantified DNA using a NanoDrop ${ }^{\oplus}$ ND-1000 Spectrophotometer at $260 \mathrm{~nm}$. We genotyped all individuals at up to 10 polymorphic microsatellite loci (C. alani: Oligr1, 3, 4, 6, 7, 8, 10, 13, 15, 19; C. whitakeri: Oligr 2, 4, 6, 7, 8, 10, 14, 15, 17; O. suteri: Oligr 1, 2, 4, 8, 10, 13; Berry et al. 2003) adapted with 5' M-13 tags (Schuelke 2000). PCR was carried out on an Eppendorf Mastercycler thermocycler as outlined in Miller et al. (2009b, Chapter 2). PCR products were pooled for genotyping and run on an ABI3730 Genetic Analyzer (Applied Biosystems, Inc.). Allele sizes were scored manually using GENEMAPPER 3.7 (Applied Biosystems, Inc.). 


\subsubsection{Data analysis}

Prior to microsatellite analyses, we calculated the frequency of null alleles in MICROCHECKER 2.2 (Van Oosterhout et al. 2004). Three loci (Oligr3, Oligr13, and Oligr15) had a high frequency of null alleles (>0.15) in the Korapuki and Middle Island populations of $C$. alani, and were excluded from further analyses.

We divided samples into three groups: source, founders, and Korapuki Island populations. We used Middle Island for the source population for C. alani (as no animals were caught on Green Island). Departures from Hardy-Weinberg proportions were calculated in GENEPOP 4.0 (Rousset 2008). Tests of significance were combined over all loci using Fisher's combined probability test. Significance was assumed at $p<0.05$. We tested for gametic disequilibrium within each population using a Fisher's exact test for all pairwise locus comparisons in GENEPOP 4.0.

To evaluate a loss of allelic diversity after translocation of each species, we calculated allelic richness (number of alleles corrected for sample size) of each species using FSTAT 2.9 (Goudet 1995). For C. alani, we excluded the founder group from the calculation of allelic richness because of the small sample size $(n=5)$. Allele frequency mode shifts were assessed visually using the method of Luikart et al. (1998). As we had only one temporal sample, we generated estimates of the effective population size $\left(\mathrm{N}_{\mathrm{e}}\right)$ of each translocated population using the linkage disequilibrium method (implemented in LDNe, Waples \& Do 2008). We excluded alleles with frequencies less than 0.05 , as inclusion of rare alleles causes an upward bias in $\mathrm{N}_{\mathrm{e}}$ estimates (Waples \& Do 2008) and used $95 \% \mathrm{Cls}$ adjusted using the jackknife method. To test for differences in allele frequencies, we conducted pairwise analyses of molecular variance (AMOVA, Excoffier et al. 1992) in GENALEX 6.1 (Peakall \& Smouse 2006) between the source population, founders, and the Korapuki population for C. whitakeri and O. suteri.

We used the program VORTEX 9.92 (Lacy 1993) to model the expected loss of heterozygosity over 10 generations under several management scenarios in each of the translocations to Korapuki Island and the proposed second-order translocations. VORTEX assigns two unique alleles to each founder; in each iteration, these hypothetical alleles are 'dropped' through the simulated population from parents to offspring according to Mendelian inheritance. At the end of each iteration, the proportion of heterozygosity that 
remains in the population is calculated. We ran 10,000 iterations for each scenario to provide estimates of the loss of heterozygosity given the stochastic natures of inheritance and population growth.

We modelled the loss of heterozygosity and the number of years to reach carrying capacity in a first-order translocation with the intended founder groups (30 founders) and the true founder group size on Korapuki Island for each species (to account for first-year survivorship, Table 4.1). To determine whether supplementation of the populations on Korapuki Island would reduce the loss of heterozygosity, we also modelled several potential supplementation strategies: (1) 30 adults in 2015, (2) 10 adults in 2015, and (3) 10 adults in three successive years (starting in 2015; total of 30 supplemented). For each strategy, we used three different sex ratios (all male, all female, or equal sex ratio), because different sexes have different influences on population growth rates and ultimately how well genetic diversity is maintained.

As Korapuki Island is proposed as a source for a second-order translocation, we modelled two strategies for the harvest of 30 animals from Korapuki Island in the year 2015 for each species: (1) equal sex ratio and (2) 1M:2F. To evaluate the effect of a serial bottleneck, we also modelled loss of heterozygosity in the second-order translocation in 2015 (under scenarios of no supplementation) and translocation in 2025 (i.e. 10 years after supplementation).

\subsection{Results}

All populations were in Hardy-Weinberg proportions at all loci $(p>0.05)$. Significant gametic disequilibrium $(p<0.05)$ was detected in five of 15 pairwise locus comparisons in the O. suteri population on Korapuki Island after sequential Bonferroni correction. No significant gametic disequilibrium was detected in any other population.

Mean allelic richness and heterozygosity was lower in the translocated populations on Korapuki Island than the natural populations for each species (Table 4.2). There was no mode shift of allele frequency distributions in any group surveyed (Figure 4.2), but the mean proportion of rare alleles (frequency $<0.1$ ) was lower in the translocated population than the natural population for each species. On Korapuki Island, $\mathrm{N}_{\mathrm{e}}$ was estimated at 14.1 for C. alani, 10.6 for C. whitakeri, and 47.5 for O. suteri. Levels of 
differentiation between the source and translocated populations of $C$. whitakeri and $O$. suteri were low but significant $\left(\Phi_{\mathrm{PT}}=0.022\right.$ and $\Phi_{\mathrm{PT}}=0.021$, respectively, $p<0.01$ for both species), but founder groups were not different from the source or translocated populations for either species $\left(\Phi_{\mathrm{PT}}=0.000-0.010, p>0.05\right)$.

Table 4.2 Sample sizes, heterozygosity $\left(H_{E}\right)$, and allelic richness measured in each skink population. $\mathrm{N}_{\mathrm{e}}$ estimates $(95 \% \mathrm{Cl})$ and the predicted and actual proportion of $\mathrm{H}_{\mathrm{E}}$ of the source population remaining in the translocated populations on Korapuki Island in the sampling year (2006).

\begin{tabular}{|c|c|c|c|}
\hline & Cyclodina alani & Cyclodina whitakeri & Oligosoma suteri \\
\hline \multicolumn{4}{|l|}{ Sample Sizes } \\
\hline Source & $30^{1}$ & 32 & 53 \\
\hline Founders ${ }^{2}$ & 5 & 16 & 30 \\
\hline Korapuki $^{3}$ & 36 & 28 & 168 \\
\hline No. loci used & 7 & 9 & 6 \\
\hline \multicolumn{4}{|l|}{$H_{E}$} \\
\hline Source & $0.619^{1}$ & 0.622 & 0.821 \\
\hline Founders ${ }^{2}$ & 0.363 & 0.597 & 0.799 \\
\hline Korapuki $^{3}$ & 0.540 & 0.575 & 0.814 \\
\hline \multicolumn{4}{|l|}{ Allelic richness } \\
\hline Source & $6.00^{1}$ & 7.23 & 9.42 \\
\hline Founders ${ }^{2}$ & na & 7.08 & 8.82 \\
\hline Korapuki $^{3}$ & 3.76 & 5.74 & 8.61 \\
\hline $\mathrm{N}_{\mathrm{e}}$ on Korapuki ${ }^{2}$ & $14.1(6.7-29.3)$ & $10.6(8.0-14.2)$ & $47.9(38.3-60.4)$ \\
\hline \multicolumn{4}{|l|}{ Proportion $\mathrm{H}_{\mathrm{E}}$} \\
\hline Predicted & 0.956 & 0.969 & 0.975 \\
\hline Actual $^{3}$ & $n a^{4}$ & 0.924 & 0.990 \\
\hline \multicolumn{4}{|c|}{$\begin{array}{l}{ }^{1} \text { Middle Island was used because no animals were caught in the tru } \\
\text { (Green Island) } \\
{ }^{2} \text { Founders recaptured in the sampling year or sampled at release } \\
{ }^{3} \text { Excluding founders }\end{array}$} \\
\hline
\end{tabular}

\subsubsection{Effects of translocation and supplementation modelled in VORTEX}

The populations of $C$. alani, C. whitakeri, and O. suteri on Korapuki Island are likely to reach carrying capacity after 76,148 , and 35 years $(\sim 5,15$, and 3.5 generations) respectively. Ten generations after release, populations on Korapuki are likely to retain $89.9-96.4 \%$ of the heterozygosity of the source population (Figure 4.3). As less than half of the intended 
number of founders was used for translocation of $C$. alani (14 rather than 30 skinks), this population is likely to have the greatest loss of heterozygosity after 10 generations (10.1\%), despite reaching carrying capacity relatively quickly. Had the intended founder group size been translocated, this population should have retained $\sim 96.6 \%$ of the original heterozygosity after 10 generations and reached carrying capacity after 60 years ( 4 generations, Table 4.3).
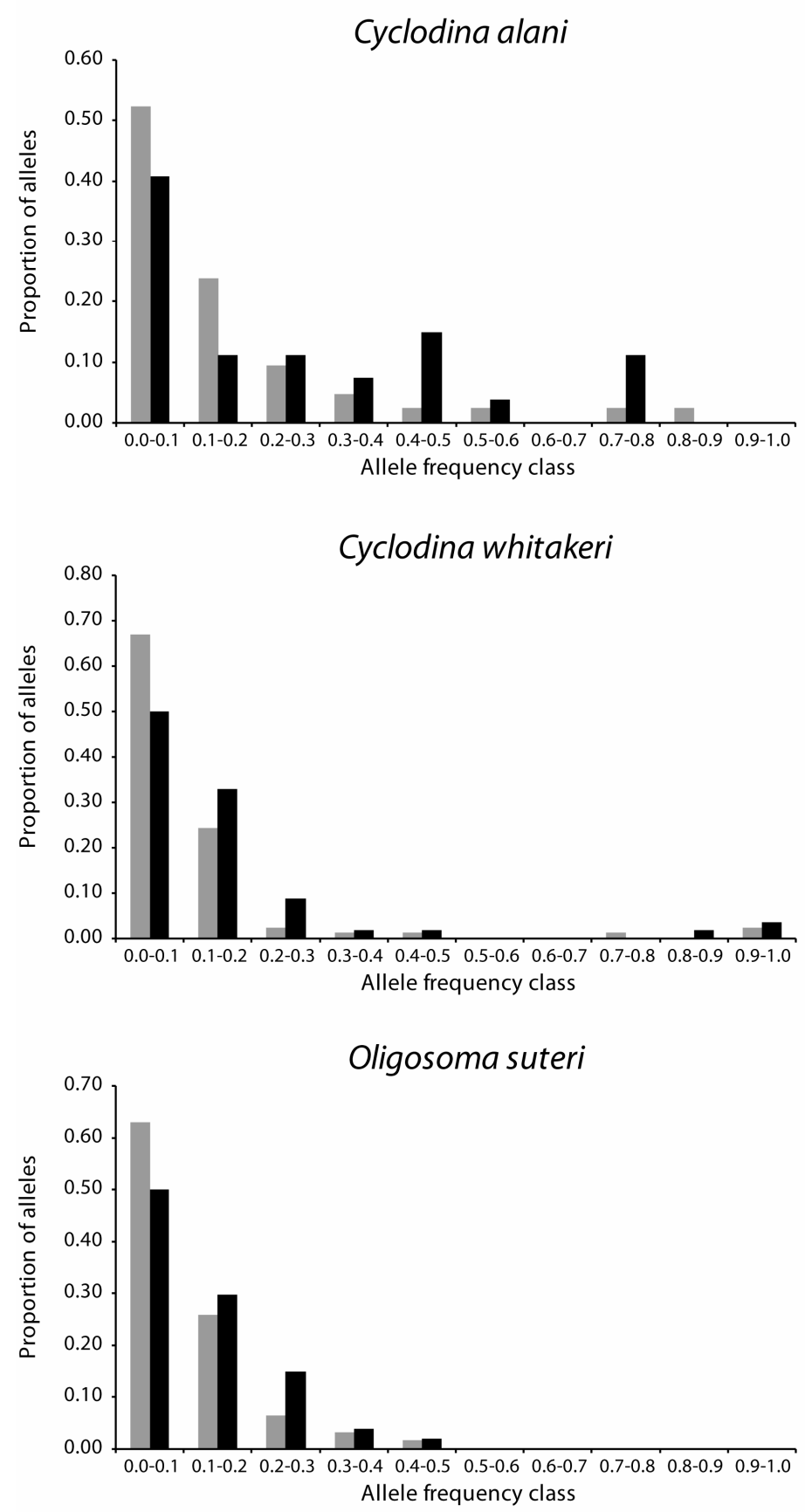

Figure 4.2 Allele frequency distribution in skink populations on Korapuki Island (black bars) and in the source populations (grey bars). For Cyclodina alani, allele frequency distributions are for Middle Island, because no skinks were caught on the source island. No population showed a significant mode shift, but rare alleles were less common in the translocated population for each species. 

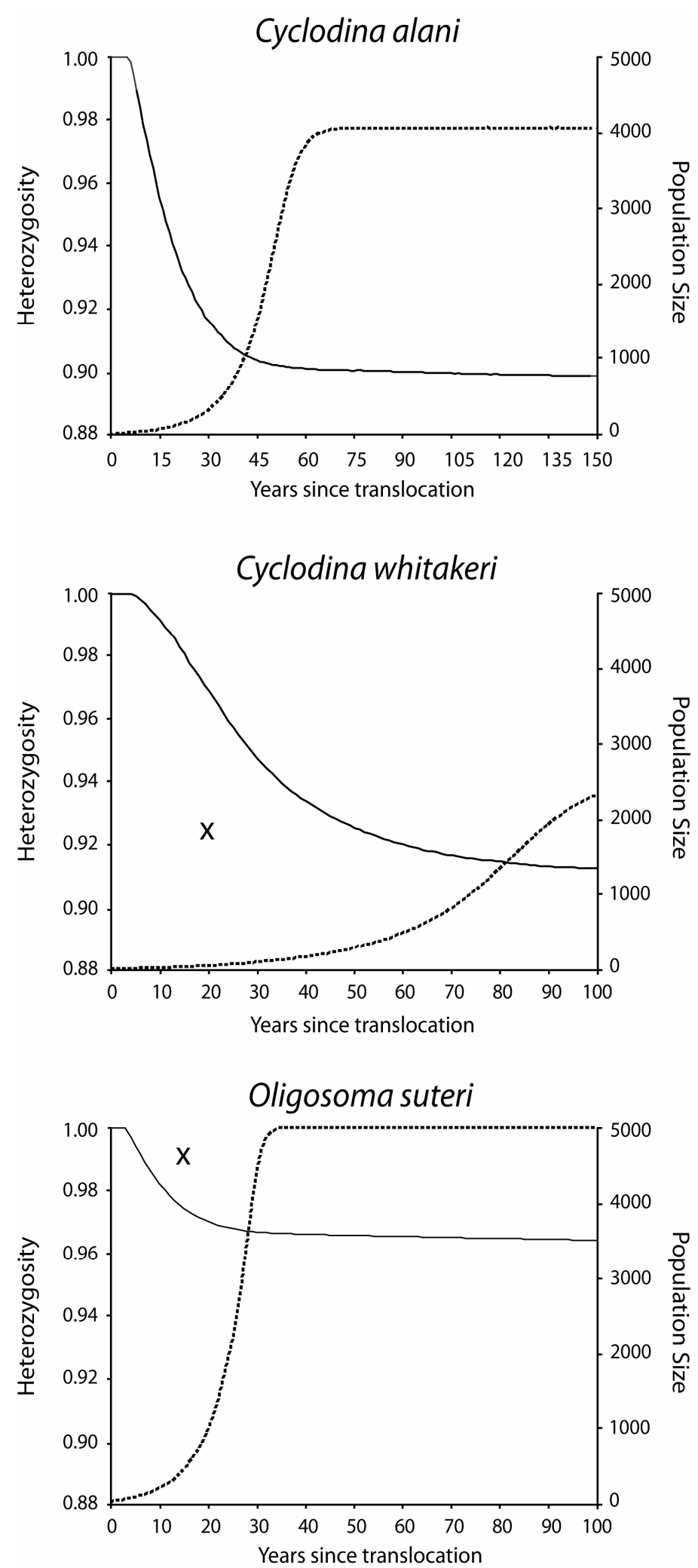

Figure 4.3 Comparison between the predicted loss of heterozygosity (solid line) and population size (broken line) over 10 generations for each translocated species on Korapuki Island. Differences in scale for each species reflect different generation intervals (Cyclodina alani: $~ 15$ years, C. whitakeri and Oligosoma suteri: $\sim 10$ years). The actual amount of heterozygosity of the source populations retained for $C$. whitakeri and O. suteri are shown with an $\mathrm{X}$ (not calculated for $C$. alani because no animals were caught in the source population). 
For O. suteri, none of the supplementation scenarios modelled resulted in a greater retention of heterozygosity or shorter time to reach carrying capacity. However, up to 93.6 and $95.7 \%$ heterozygosity of the source could be retained in the populations of $C$. alani and C. whitakeri, respectively after 10 generations by supplementation (Table 4.3). For both species, the best option for minimising the loss of genetic diversity is a single introduction of 30 animals of equal sex ratio; under this scenario, the populations should reach carrying capacity after 68 (C. alani) and 111 (C. whitakeri) years ( 4.5 and 11 generations, respectively). The introduction of 10 animals (equal sex ratio) over each of three consecutive years gave similar results. The amount of heterozygosity retained was similar when all male or all female C. alani were released (Table 4.3). However, the addition of 30 male $C$. whitakeri resulted in the retention of less heterozygosity (94.6\%) than addition of either 30 females (95.3\%) or 15 animals of both sexes (95.7\%, Table 4.3$)$.

Table 4.3 Predicted proportion of source heterozygosity $\left(H_{E}\right)$ retained after 10 generations and the time taken to reach carrying capacity $(K)$ in the first-order translocation (Korapuki Island). Each translocation was designed to have 30 founders, but true founder groups were 14 for Cyclodina alani and 28 for $C$. whitakeri. Supplementation and harvest scenarios (respectively) were all modelled for the year 2015.

\begin{tabular}{|c|c|c|c|c|c|c|}
\hline & \multicolumn{2}{|c|}{ Cyclodina alani } & \multicolumn{2}{|c|}{ Cyclodina whitakeri } & \multicolumn{2}{|c|}{ Oligosoma suteri } \\
\hline & $\mathrm{H}_{\mathrm{E}}$ & Years to $\mathrm{K}$ & $\mathrm{H}_{\mathrm{E}}$ & Years to $\mathrm{K}$ & $\mathrm{H}_{\mathrm{E}}$ & Years to $\mathrm{K}$ \\
\hline \multicolumn{7}{|l|}{ No. of founders } \\
\hline Intended (30) & 0.965 & 59 & 0.931 & 140 & 0.964 & 35 \\
\hline True (14-30) & 0.899 & 76 & 0.913 & 148 & 0.964 & 35 \\
\hline \multicolumn{7}{|l|}{ Supplementation } \\
\hline $30^{1}$ & 0.936 & 68 & 0.957 & 111 & 0.966 & 35 \\
\hline $30 \mathrm{~F}$ & 0.932 & 64 & 0.953 & 98 & 0.966 & 34 \\
\hline $30 M$ & 0.931 & 83 & 0.946 & 144 & 0.966 & 35 \\
\hline $10^{1}$ & 0.915 & 72 & 0.934 & 123 & 0.965 & 35 \\
\hline $10 \mathrm{~F}$ & 0.914 & 72 & 0.934 & 116 & 0.965 & 35 \\
\hline $10 \mathrm{M}$ & 0.913 & 70 & 0.929 & 148 & 0.965 & 35 \\
\hline $10^{1} \times 3$ years $^{2}$ & 0.934 & 68 & 0.955 & 112 & 0.965 & 34 \\
\hline $10 \mathrm{~F} \times 3$ years $^{2}$ & 0.930 & 65 & 0.952 & 100 & 0.965 & 34 \\
\hline $10 \mathrm{M} \times 3$ years $^{2}$ & 0.930 & 75 & 0.945 & 144 & 0.965 & 35 \\
\hline \multicolumn{7}{|l|}{ Harvest } \\
\hline $1: 1^{3}$ & 0.893 & 87 & 0.892 & 182 & 0.964 & 35 \\
\hline $1: 2^{3}$ & 0.892 & 98 & 0.885 & 189 & 0.964 & 35 \\
\hline
\end{tabular}




\subsubsection{Effects of serial translocation modelled in VORTEX}

By including a harvest of skinks from Korapuki Island, we simulated the effects of the proposed second-order translocation of each species (Table 4.3). Harvesting O. suteri had no effect on the amount of heterozygosity retained on Korapuki Island after 10 generations on Korapuki Island or the time it took to reach carrying capacity, but slightly less heterozygosity was retained when C. alani were harvested (equal sex ratio $=89.3 \%$, $1 \mathrm{M}: 2 \mathrm{~F}=89.2 \%)$ than would be expected without harvest $(89.9 \%)$. For $C$. whitakeri, the population on Korapuki Island retained less heterozygosity under harvest scenarios (equal sex ratio $=89.2 \%, 1 \mathrm{M}: 2 \mathrm{~F}=88.5 \%$ ) than in the scenario without harvest $(91.3 \%)$. Harvest of C. alani and C. whitakeri (equal sex ratios) increased the time it takes to reach carrying capacity to 87 and 182 years ( 6 and 18 generations), respectively.

A second-order translocation of $O$. suteri would retain approximately $93.2 \%$ of the heterozygosity from the source population after 10 generations (a 3.2\% decline of heterozygosity relative to the harvested Korapuki Island population, Table 4.4). Secondorder translocations of C. alani and C. whitakeri would retain $89.8 \%$ and $88.7 \%$ of the heterozygosity of the source populations respectively (i.e. declines of $<0.5 \%$ relative to the harvested populations on Korapuki Island). By supplementing the populations on Korapuki Island with 30 animals of equal sex ratio, the retention of heterozygosity over 10 generations in a second-order translocation relative to the wild population could increase to $91.2 \%$ (C. alani), $90.1 \%$ (C. whitakeri), and $93.4 \%$ (O. suteri, Table 4.4).

Table 4.4 Predicted proportion of source heterozygosity retained in a second-order translocation (e.g. translocation of 30 skinks from Korapuki Island) after 10 generations. Predictions are shown for the second-order translocation from Korapuki Island in 2015 (no supplementation), or in 2025 (10 years after supplementation of the population on Korapuki Island).

\begin{tabular}{lccc}
\hline & Cyclodina alani & Cyclodina whitakeri & Oligosoma suteri \\
\hline No supplementation & 0.898 & 0.887 & 0.932 \\
& & & \\
Strategy for supplementation of & & & \\
Korapuki Island & & & \\
30 (1M:1F) & 0.912 & 0.901 & 0.934 \\
30F & 0.908 & 0.898 & 0.934 \\
$30 \mathrm{M}$ & 0.909 & 0.899 & 0.934 \\
$10(1 \mathrm{M}: 1 \mathrm{~F})$ & 0.894 & 0.887 & 0.933 \\
\hline
\end{tabular}




\subsection{Discussion}

Translocation of three species of skink to Korapuki Island caused losses of heterozygosity and allelic diversity. The population of $C$. whitakeri on Korapuki Island retained a smaller proportion of the heterozygosity of its source population than predicted by models, but the population of $O$. suteri retained a greater proportion. Supplementation of translocated populations on Korapuki Island reduces the loss of heterozygosity over 10 generations in C. whitakeri and C. alani, but not O. suteri. Second-order translocation of 30 C. whitakeri from Korapuki Island would reduce the proportion of heterozygosity retained on Korapuki Island, while harvests of $C$. alani or O. suteri would have little to no effect. As expected, second-order translocations of 30 skinks from Korapuki Island would retain less diversity than a first-order translocation of 30 animals for all three species. Surprisingly, second-order translocations of $C$. alani and C. whitakeri would retain similar amounts of heterozygosity after 10 generations as the harvested populations on Korapuki Island.

The strength of a bottleneck influences the amount of genetic diversity initially retained, but the duration of the bottleneck will affect how well that diversity is maintained over many generations (Allendorf \& Luikart 2007). Thus, founder group size dictates how much diversity is represented in a translocated population, but the rate of population expansion affects how well that diversity is maintained. With identical founder group sizes for each species, translocated populations of $C$. whitakeri (i.e. the species with the lowest reproductive output) loses more genetic diversity over 10 generations than $C$. alani or $O$. suteri. Therefore, translocations of species with low reproductive output may require more founders to meet genetic goals for management. As species with low reproductive output are also generally rarer, a potential dilemma arises between the lower availability of founders for a translocation and the greater number required to meet the genetic goals of management. Similarly, rarer species may have increased extinction risks after translocation and thus require larger founder groups (Towns \& Ferreira 2001).

\subsubsection{Do models of genetic diversity at neutral loci represent losses of functional diversity?}

Models of neutral genetic variation have been questioned, as they may have only a limited ability to predict the effects of a bottleneck on quantitative (polygenic) variation (Reed \& Frankham 2001). Where quantitative variation is purely additive, it is lost at the 
same rate as neutral heterozygosity, yet many polygenic traits associated with fitness often deviate from additivity and have large amounts of dominance and epistatic genetic variance (Crnokrak \& Roff 1995). Empirical studies of the effect of bottlenecks on quantitative variation are somewhat contradictory; after a reduction in population size, quantitative variation may be maintained (Van Oosterhout \& Brakefield 1999), lost (Gilligan et al. 2005), or even gained (Saccheri et al. 2001) by the conversion of dominance and epistatic variation to additive variation (Wang et al. 1998). However, the loss of genetic variation at some functional loci (e.g. genes of the major histocompatibility complex, MHC) after a bottleneck is comparable to that lost at neutral markers (Miller et al. 2008), and genetic drift in small populations generally outweighs balancing selection at MHC loci (Miller \& Lambert 2004; Campos et al. 2006, but see Aguilar et al. 2004). Thus, models based on neutral markers can provide an indicator of genetic diversity at some functional loci, and can be used to evaluate the merit of various management actions.

\subsubsection{How much genetic diversity is enough?}

The genetic goals for management of translocated populations will influence how many founders should be released, whether additional releases are required, and whether serial translocation is a viable option, yet specific targets for the maintenance of genetic diversity are rarely included in recovery programs for species in New Zealand (Grueber \& Jamieson 2008). Commonly cited targets are $90-95 \%$ heterozygosity over 100-200 years (Soulé et al. 1986; Lacy 1987; Allendorf \& Ryman 2002), but it is generally agreed that the more heterozygosity retained, the better (Franklin \& Frankham 1998; Lynch \& Lande 1998). In many captive breeding programs, it is possible to equalise founder contributions to maximise the retention of genetic diversity and reduce inbreeding (but see Moore et al. 2008). However in translocated populations, it is not possible to manage breeding pairs. Although predictions can be made about the retention of genetic diversity before translocation, the stochastic nature of inheritance and the variability of population growth mean that continued genetic monitoring is required. Genetic monitoring during the period of population growth after translocation can give insight into how equally founders are represented and whether populations will meet genetic goals for management. 


\subsubsection{Genetic impacts of first- and second- order translocation}

In monitoring three species of skink 14-18 years after translocation, we found that genetic diversity in the populations of $C$. alani and C. whitakeri is lower than predicted. We therefore recommend that some restorative genetic management be undertaken. The predicted losses of genetic diversity in each of the skink populations on Korapuki Island after 10 generations fall within the lower targets for maintenance of heterozygosity. These predictions are likely to still be overestimates, as the actual heterozygosity retained over 14-18 years was lower than predicted for at least one species (C. whitakeri), and probably a second (C. alani, but we could not directly evaluate heterozygosity in the source population). The losses are not as large as predicted for other species (e.g. takahe, Porphyrio hochstetteri, Grueber \& Jamieson 2008), but if the actual losses of heterozygosity over 10 generations for $C$. whitakeri and C. alani are greater than predicted, they would fall well outside the target values.

The effective population sizes of each species on Korapuki Island relative to the number of founders released reflect how well diversity was maintained over 14-18 years. The population of $O$. suteri retained more heterozygosity than predicted over 14 years and had a larger $\mathrm{N}_{\mathrm{e}}$ than the number of animals released, because the release of gravid females was successfully used to maximise genetic diversity in this population (Miller et al. in review-b, Chapter 3). On the other hand, the population of C. whitakeri had an $\mathrm{N}_{\mathrm{e}}$ almost three times smaller than the number of founders released (and half the number of founders that survived translocation). This population also lost more heterozygosity over 18 years than expected.

Our models show that supplementation while translocated populations are small could be used to slow the long-term loss of genetic diversity (e.g. C. whitakeri and C. alani). However, when populations expand rapidly after translocation (e.g. O. suteri), supplementation has little impact on the amount of heterozygosity maintained over 10 generations. Supplementation increases the rate of population growth, thus reducing the time required to reach carrying capacity for C. alani and C. whitakeri (by 8 and 37 years, respectively), but not for $O$. suteri. Similarly, harvest for a second-order translocation has severe impacts on small populations, but little to no impact on populations that have expanded rapidly. 
It must be noted that the scenarios modelled assume that all supplemented (or harvested) individuals survive translocation. Low survivorship after release must therefore be accounted for by increasing the number of animals supplemented (or harvested). In the case of supplementation of the Korapuki Island population with $30 \mathrm{C}$. whitakeri, up to 50 animals would need to be released in order to account for only $60 \%$ survivorship (Towns \& Ferreira 2001). However, increasing the number of animals harvested for a second-order translocation would simultaneously accelerate the loss of genetic diversity on Korapuki Island by increasing the duration of the bottleneck.

Serial translocation of skinks from Korapuki Island is proposed to reduce impacts on the wild sources (Middle and Green Islands). Whilst this goal is valid, and certainly wild populations should not be harmed by the removal of animals, serial translocation of species with slow population growth rates may be undesirable. If C. alani or C. whitakeri were harvested from Korapuki Island, neither translocated population would meet targets for the maintenance of genetic diversity. Even after supplementing populations on Korapuki Island, genetic diversity in the second-order translocations would be considerably lower than a first-order translocation of the same size. This creates a conflict between the goals for managing the wild and translocated populations: establishing new populations that meet recovery goals, whilst minimising disturbance to the wild populations. Similar conflicts arise when translocations are designed using animals from captivity: using genetically over-represented animals as founders is the best option for captive programmes, but the worst for the translocated population (Earnhardt 1999). When such conflict arises, the management options must be evaluated in light of the demographic and genetic goals for species recovery. Cyclodina alani and C. whitakeri have six and three remaining natural populations, respectively. Therefore, considerable pressure exists for further translocations, but postponing second-order translocations until the populations on Korapuki Island are larger may be a better option for management in the long-term.

Lastly, our study underlines the need for thorough population surveys in proposed source populations prior to translocation. As we were unable to find any C. alani on Green Island (the source for translocation to Korapuki Island), it is likely that the population on Green Island was very small, and that the harvest of animals for translocation had negative demographic consequences. Although supplementation would help to slow the loss of genetic diversity in the population of $C$. alani on Korapuki Island, animals could not 
feasibly be sourced from Green Island, and supplementation would require mixing populations. As Middle, Green, and Korapuki Islands were connected within the last 6,000 years (Towns 1994), it is unlikely that populations on Green and Middle Islands are locally adapted. Supplementing the translocated population on Korapuki Island with C. alani from Middle Island would mimic the historic relationships between populations.

In conclusion, rapid population growth is essential for maximising the retention of genetic diversity and reducing the effects of inbreeding depression. Reducing the duration of the bottleneck after translocation should be a major consideration when designing translocations, and our research shows that actions that slow population growth (e.g. harvest from a relatively small population for a second order translocation) should be avoided. Supplementation of translocated populations will have the greatest effect during the period of population growth, as new animals will aid in rapid recovery from a bottleneck. 


\section{CHAPTER FIVE}

\section{How do reproductive skew and founder group size affect genetic diversity in reintroduced populations?}

\subsection{Abstract}

Reduced genetic diversity results in short-term decreases in fitness and reduced adaptive potential, which leads to an increased extinction risk. Therefore, maintaining genetic variation is important for the short- and long-term success of reintroduced populations. Here we evaluate how founder group size and variance in male reproductive success influence the long-term maintenance of genetic diversity after reintroduction. We used microsatellite data to quantify the loss of heterozygosity and allelic diversity in the founder groups from three reintroductions of tuatara (Sphenodon spp.), the sole living representatives of the reptilian order Rhynchocephalia. We then estimated the maintenance of genetic diversity over 10 generations (400 years) using population viability analysis. Reproduction of tuatara is highly skewed, with as few as $30 \%$ of males mating across years. Predicted losses of heterozygosity over 10 generations were low (1-14\%), and as predicted, populations founded with more animals retained a greater proportion of the heterozygosity and allelic diversity of their source populations and founder groups. Greater male reproductive skew led to a greater loss of genetic diversity over 10 generations, but only accelerated the loss of genetic diversity at small population size ( $<250$ animals). A reduction in reproductive skew at low density may facilitate the maintenance of genetic diversity in small reintroduced populations. However, if reproductive skew is high and density-independent, larger founder groups could be released to achieve genetic goals for management.

\subsection{Introduction}

Maintaining genetic diversity is a common goal for species management, yet routine management actions such as reintroductions may impose genetic bottlenecks. After a bottleneck, reduced genetic diversity can result in short-term decreases in fitness (inbreeding depression, Jamieson et al. 2007), increased extinction risk (Saccheri et al. 1998), and reduced evolutionary potential (Frankham 1999; 2005). Effective management 
of genetic diversity in reintroduced populations is therefore important for both shortand long-term success (Fitzsimmons et al. 1997; Armstrong \& Seddon 2008), but founder group sizes are often small (Griffith et al. 1989), and reintroduced populations often have lower genetic diversity than their sources at neutral and functional loci (Williams et al. 2000; Miller \& Lambert 2004). Predicting how management actions affect genetic diversity in reintroduced populations is therefore a priority both for species recovery (e.g. Towns 1999) and in the broader field of reintroduction biology (Armstrong \& Seddon 2008).

Several factors will differentially affect the maintenance of genetic diversity in reintroduced populations. First, while reintroductions, particularly those with small founder groups, are likely to cause a genetic bottleneck and promote losses of genetic diversity, losses of diversity could be minimised when populations expand rapidly after reintroduction (Chapter 4). Second, unequal reproduction (i.e. founder representation in the offspring) may lead to a smaller effective population size, but a high degree of generation overlap may minimise this effect (Nunney 1993). Lastly, density-dependence in the mating and social systems (Kokko \& Rankin 2006) may accelerate or slow the loss of genetic diversity.

In many species, mating success is unequal among individuals (Emlen \& Oring 1977), and this variance can lead to decreases in effective population size (Anthony \& Blumstein 2000). Indeed, highly polygynous mating systems, including harem and dominance polygyny, can lead to very low effective population sizes when generations are nonoverlapping (Nunney 1993; Parker \& Waite 1997). As the degree of generation overlap increases, the impact of varying reproductive success is harder to quantify. As the generation interval increases, the reduction in the effective population size should be minimised (Nunney 1993), yet at small population sizes, even a slight reduction in the effective population size could result in rapid genetic drift. However, the effect of variation in male reproductive success is unclear when population size is increasing (e.g. after reintroduction).

Mating systems and the determinants of male reproductive success are often densitydependent (Kokko \& Rankin 2006). When males interact locally, for example, dominant males may thwart mating attempts of nearby subordinate males. When male-male competition increases with density, theory predicts that a smaller proportion of males 
will successfully mate at higher density (Kokko \& Rankin 2006). However, this relationship does not always hold. Large male seed bugs (Neacorphyus bicrucis) defend territories against smaller males in order to acquire mates. At high density, however, these large males are less likely to monopolize territories, and smaller males are more likely to mate (McLain 1992). At very high densities, the energetic costs of site defence outweigh the reproductive benefits (Brown 1969; Emlen \& Oring 1977), and males may abandon territoriality altogether (Maher \& Lott 2000). Thus, variation in male reproductive success may change at differing population densities.

We assessed how unequal reproductive success and founder group sizes influence the long-term maintenance of genetic diversity in reintroduced populations. We use the term reproductive skew to specify the percentage of males that do not mate during their lifetime (i.e. those excluded from mating by dominant males).

\subsubsection{The mating system of a rare reptile}

Tuatara (Sphenodon spp.) are threatened reptiles endemic to New Zealand, and are the sole representatives of the ancient reptilian order Rhynchocephalia (Cree \& Butler 1993). They are medium-sized, territorial, and extremely long-lived (possibly 100 years, Dawbin 1982; Cree 1994). Females breed asynchronously and are reproductively active, on average, once every four years. Males are capable of reproduction every year (Cree et al. 1992). Tuatara are primarily seasonally monogamous, with low levels of polygyny and polyandry $(<10 \%)$. However, they are both polygynous and polyandrous across seasons (Moore et al. in review). Males defend territories that overlap with an average of four females, not all of which are receptive in any one year (Moore et al. in press). Large male body size is the primary determinant of reproductive success, but mate choice is also influenced by disassortative mating at MHC loci (major histocompatability complex, Miller et al. 2009a). Large males occupy areas where females are most dense, and they interfere with courtships and prevent mating of smaller males (Moore et al. in press). Large body size in tuatara is most likely influenced by resource availability rather than genetic factors (Miller et al. 2009a). By out-competing small males for food, large males may also limit the growth of small males and thus lifetime mating opportunities. Male reproductive skew is high, with as few as $30 \%$ of males obtaining mates across years (i.e. $70 \%$ reproductive skew), but this relationship may change with density. In habitats with lower tuatara density, males may be less able to defend receptive females whose 
territories overlap their own, as the average body size of males that successfully mate is smaller (Moore et al. in review). Further, in a captive population of eight tuatara, three of the four males successfully sired offspring over 15 years. However, the largest male sired $80 \%$ of all offspring (Moore et al. 2008).

\subsubsection{Conservation of tuatara by reintroduction}

Tuatara were once found throughout mainland New Zealand, but are now restricted to $\sim 30$ offshore islands that are free of introduced mammalian predators (Cree \& Butler 1993). Between 1995 and 2008, tuatara were reintroduced to 12 sites. Four natural populations of tuatara were reduced to very small sizes because of predation by introduced mammals. All individuals captured on these islands were taken into captivity, and captive-bred and reared offspring have been reintroduced to their original islands, which were cleared of introduced mammals. The other eight reintroductions were from islands to areas within their former range now cleared of mammalian predators. A total of five source populations were used, but only a single source was used to found each reintroduction. Five reintroductions were sourced from two islands: Stephens Island (2 reintroductions) and North Brother Island (3 reintroductions). Stephens Island (150 ha, $40^{\circ} 40^{\prime} \mathrm{S}, 174^{\circ} 00^{\prime} \mathrm{E}$ ) has the largest population of tuatara (30-50,000 individuals, Newman 1987) and high levels of genetic diversity at both neutral and functional loci (Miller et al. 2007; Hay \& Lambert 2008). North Brother Island ( $4 \mathrm{ha}, 41^{\circ} 07^{\prime} \mathrm{S}, 174^{\circ} 27^{\prime} \mathrm{E}$ ) has a small population size ( 350 adults, Newman 1877; Nelson et al. 2002b) and very low levels of genetic diversity at neutral and functional loci (Miller et al. 2008).

Initial survival of adults in the first year after reintroduction is high (at least $80 \%$, and probably higher, McKenzie 2007), and tuatara show rapid increases in both body size and condition (Nelson et al. 2002a; McKenzie 2007). However, successful reproduction has only been confirmed in three reintroduced populations, as hatchling and juvenile tuatara are extremely difficult to detect. Because of the extreme longevity, long reproductive interval, and cryptic behaviour of tuatara, demographic and genetic models are important for predicting the effects of management actions.

We used three well-monitored reintroduced populations of tuatara to measure losses of genetic diversity in the founder groups and to predict the maintenance of that diversity over 10 generations. In order to determine the effects of reproductive skew and founder 
group sizes on genetic diversity in reintroduced populations of tuatara, we used microsatellite data from source populations with high and low levels of genetic diversity to quantify the initial genetic bottleneck (i.e. the loss of alleles in the founder groups), and to predict the loss of heterozygosity and allelic diversity over 10 generations with different founder group sizes.

\subsection{Methods}

\subsubsection{Microsatellite genotyping}

Tuatara were reintroduced from North Brother Island to Titi and Matiu/Somes Islands in 1995 and 1998 (with 68 and 55 founders, respectively, Figure 5.1). In 2005, 70 tuatara were reintroduced from Stephens Island to Karori Wildlife Sanctuary, a fenced reserve on the New Zealand mainland cleared of mammalian predators; this population was supplemented with an additional 130 tuatara in 2007. We sampled DNA from tuatara in both the source and reintroduced populations (Figure 5.1), by taking buccal swabs or blood samples. We randomly sampled 246 tuatara from Stephens Island and 55 tuatara from North Brother Island. Animals reintroduced to Karori Wildlife Sanctuary were sampled at the time of translocation, and animals on Titi and Matiu/Somes Islands were sampled between December 2006 and March 2007 (Austral summer). Only a subset of tuatara in reintroduced populations are caught in any one season, due to their cryptic behaviour and the short duration of most survey trips (Nelson et al. 2002a). Therefore, sample sizes from Titi and Matiu/Somes Islands are smaller than the number of founders released (Figure 5.1).

We extracted total genomic DNA using a standard proteinase $\mathrm{K}$ phenol-chloroform protocol (Sambrook et al. 1989) followed by ethanol precipitation or using a Qiagen DNEasy kit. We genotyped all animals from North Brother Island at six polymorphic microsatellite loci (A12N, C11P, C12F, E11N, H5H and H4H, Aitken et al. 2001; Hay \& Lambert 2008). Animals from Stephens Island were genotyped at one additional locus (C2F). PCR was carried out on an Eppendorf Mastercycler thermocycler as outlined in Moore et al. (2008), and products were run on an ABI3730 Genetic Analyzer (Applied Biosystems, Inc.). Allele sizes were scored manually using GENEMAPPER 3.7 (Applied Biosystems, Inc.). 


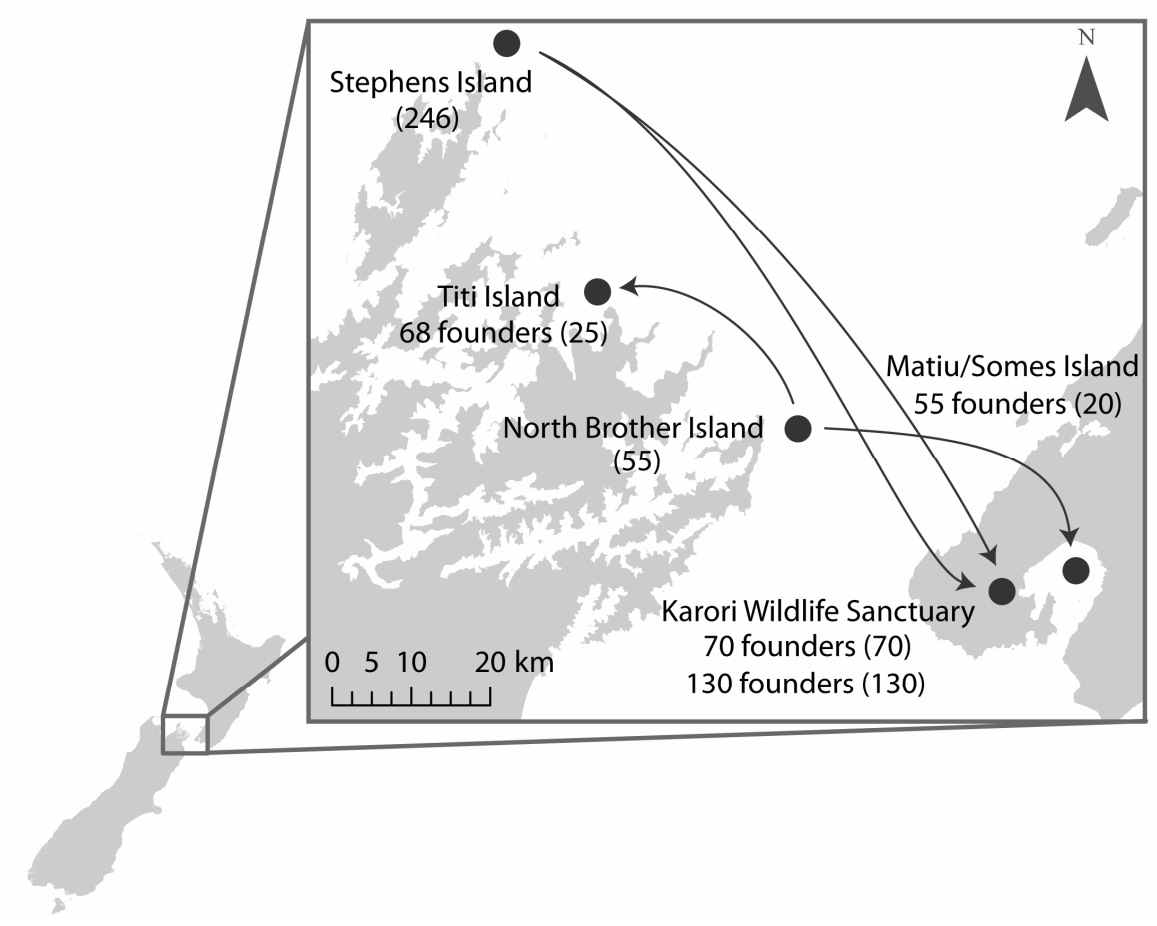

Figure 5.1 Map of Cook Strait, New Zealand, showing the source populations (North Brother and Stephens Islands) and reintroduction sites (Titi Island, Matiu/Somes Island, and Karori Wildlife Sanctuary) in this study. The number of founders released during each reintroduction is specified, and the number of individuals sampled is given in brackets.

\subsubsection{Data analysis}

Deviations from Hardy-Weinberg equilibrium (HWE) were tested in GENEPOP 4.0 (Rousset 2008). Tests of significance were combined over all loci using Fisher's combined probability test, and significance was assumed at $p<0.05$. The expected number of alleles represented $E\left(n^{\prime}\right)$ in founder groups of $N$ individuals from North Brother and Stephens Islands was calculated using the formula

$$
E\left(n^{\prime}\right)=n-\sum_{j=1}^{n}\left(1-p_{j}\right)^{2 N}
$$

where $n$ is the starting number of alleles and $p_{j}$ is the frequency of the $j^{\text {th }}$ allele (Allendorf 1986). We then compared the actual number of alleles detected in each of the reintroduced populations to that expected with the number of founders. The expected proportion of the original heterozygosity remaining in a founder group of $N$ individuals (Allendorf 1986) was calculated using the formula

$1-\frac{1}{2 N}$ 
We used VORTEX 9.92 (Lacy 1993) to model the expected loss of heterozygosity $(h)$ and allelic diversity $(A)$ over 10 generations in reintroduced populations of tuatara from Stephens and North Brother Islands (for demographic input parameters, see Table 5.1). The mean generation interval in our simulations, calculated in VORTEX from a stable age distribution, was 39.5 using our input parameters. Therefore, we ran each simulation for 400 years ( 10 generations). VORTEX assigns two unique alleles to each founder; in each iteration, these hypothetical alleles are 'dropped' through the simulated population from parents to offspring according to Mendelian inheritance. At the end of each iteration, the proportion of $h$ that remains (or $1-f$, the inbreeding coefficient) in the population is calculated using this infinite allele model. Thus, with all other demographic parameters being equal, different $h$ values result from differences in either the number of founders or carrying capacity. We used microsatellite data from Titi Island and Karori Wildlife Sanctuary to calculate allele retention when reintroductions are founded from low(North Brother Island) and high-diversity (Stephens Island) source populations. Microsatellite diversity in tuatara reflects the amount of diversity seen at MHC loci (Miller et al. 2008), which are the most variable known vertebrate genes. We ran 1,000 iterations for each scenario to provide estimates of the loss of $h$ and $A$ given the stochastic natures of inheritance and population growth.

Prior to running models of different founder group sizes and levels of reproductive skew, we tested the influence of inbreeding depression on the loss of $h$ after 10 generations in our models. We used groups of 30 adults with each of three levels of reproductive skew (70\%, 50\%, and 0\%) with no inbreeding depression and with inbreeding depression (3.14 lethal equivalents/individual, $50 \%$ due to recessive lethals). Inbreeding depression had little effect on the amount of $h$ retained after 10 generations, even at an unrealistically low carrying capacity (5000 individuals) for the population in Karori Wildlife Sanctuary $(<0.1 \%$ less $h$ retained relative to models with no inbreeding depression). Therefore, we ignored inbreeding depression in further models.

To determine how well $h$ and $A$ are maintained for 10 generations with different founder groups, we modelled four different founder group sizes for translocations from Stephens and North Brother Islands: 200 adults, 70 adults, 30 adults, and 30 juveniles. Two-hundred adults represent the maximum number of wild founders used for reintroduction; 70 adults represent the first reintroduction to Karori Wildlife Sanctuary and a similar founder group to Titi and Matiu/Somes Islands. As mainland fenced reserves are becoming more 


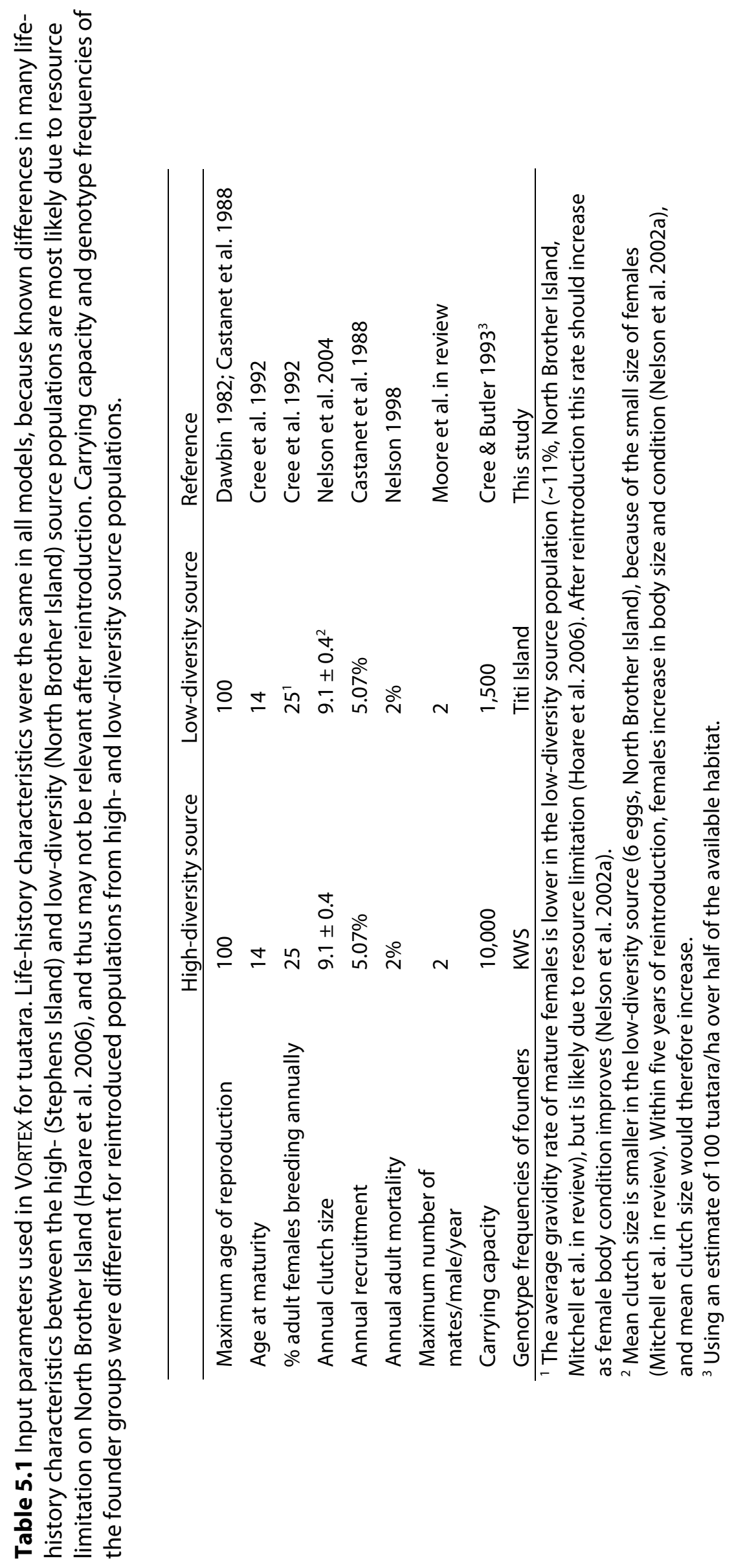


common in New Zealand, the number of tuatara reintroductions is expected to increase, and it is likely that founder groups will be comprised of $\sim 30$ adults for most of these reintroductions (P Gaze, pers. comm.). Thirty juveniles is a similar founder group as some reintroductions of captive-bred/reared populations. For each founder group, we ran three separate simulations with different levels of male reproductive skew $(70 \%, 50 \%$, or $0 \%)$. Ages of adult founders were chosen based on a stable age distribution. The age of all juvenile founders was set to five years (the age at which captive-reared tuatara are generally released).

To more thoroughly assess the effects of male reproductive skew, we ran 10 models of a reintroduction of 30 adult tuatara from both low- (North Brother Island) and highdiversity (Stephens Island) source populations. In each model, we specified a different level of male reproductive skew. We ran eight density-independent models (0-70\% reproductive skew, at $10 \%$ increments) and two density-dependent models. Reproductive skew $\left(R_{N}\right)$ at a given population size $(N)$ was directly proportional to population density in the latter models, where

$R_{N}=R_{L}+\left[\left(R_{H}-R_{L}\right) *(N / K)\right]$

and $R_{H}$ is the reproductive skew at high density, $R_{L}$ is the reproductive skew at low density, and $K$ is the carrying capacity. In the first of these models, we used $20 \%$ reproductive skew at low density and 70\% at high density (i.e. $R_{L}=20$ and $R_{H}=70$ ). In the second density-dependent model, we used $0 \%$ reproductive skew at low density and $50 \%$ at high density (i.e. $R_{L}=0$ and $R_{H}=50$ ). We based these values on data from captivity and Stephens Island (respectively), where reproductive skew is $25 \%$ at low density (Moore et al. 2008) and 70\% at high density (Moore et al. in review). To make comparisons with the density-independent models, we used 20\% skew at low density rather than 25\%. As data from captivity and Stephens Island were collected over 15 and 3 years, respectively, and may overestimate reproductive skew over the lifespan of a tuatara, we used the more conservative estimates in the second density-dependent model.

\subsection{Results}

Expected heterozygosity per locus ranged from 0.071 to 0.497 (mean $=0.406$ ) on North Brother Island and from 0.730 to 0.927 (mean 0.782) on Stephens Island. Following Bonferroni correction, only one locus ( $H 5 \mathrm{~V}$ ) deviated significantly from HWE in the North 
Brother and Stephens Island populations, but it was included in models of allelic diversity. Mean allelic diversity was 14.4 on Stephens Island and 2.3 on North Brother Island.

The proportion of alleles retained in each founder group ranged from $84.2 \%$ to $100 \%$, and was within $2 \%$ of the predicted values (Figure 5.2, Table 5.2). Groups of 30 founders from Stephens Island were predicted to retain only $70.9 \%$ of the allelic diversity of the source, but 30 founders from North Brother Island were expected to retain $99.2 \%$ of the allelic diversity on North Brother. Founder groups of 30-200 individuals represent between 98.3 and $99.8 \%$ of the original heterozygosity (Table 5.2).

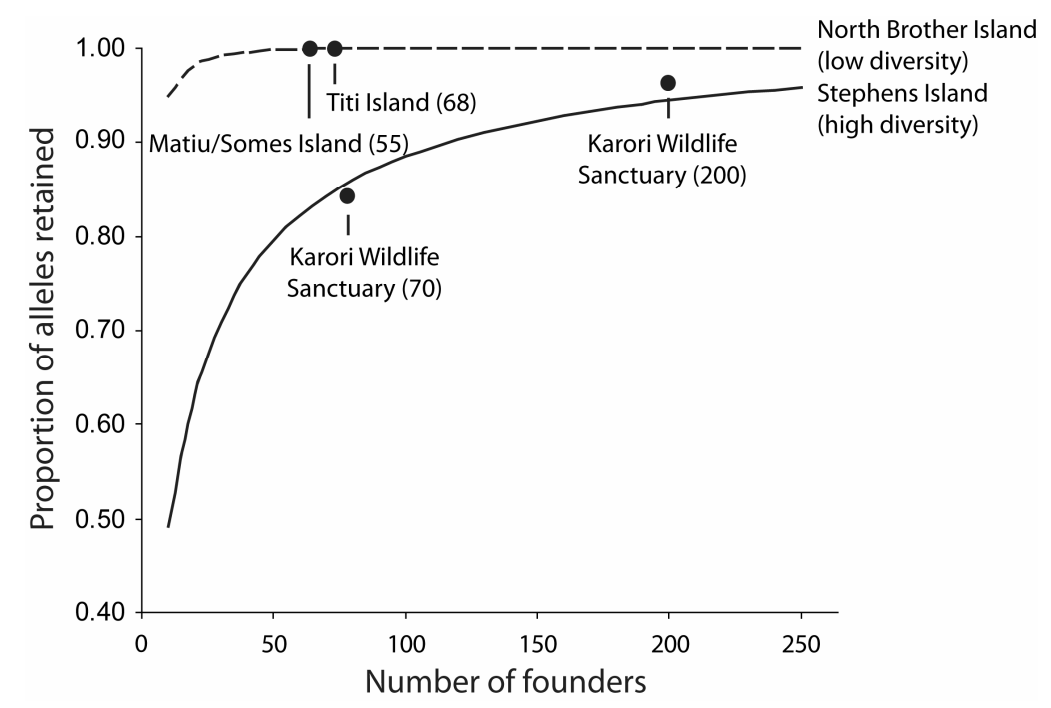

Figure 5.2 Comparison between the predicted loss of alleles with different founder group sizes, based on allele frequencies in high-diversity (Stephens Island, solid line) and low-diversity (North Brother Island, broken line) populations. The actual proportion of alleles retained in the reintroduced populations of tuatara are indicated with circles. The number of tuatara reintroduced is indicated in brackets.

Populations with larger founder groups retained more $h$ and $A$ after 10 generations for both high- and low-diversity source populations (Table 5.2). Further, populations with more founders retained a greater proportion of the $h$ and $A$ of the founders. For example, populations founded with 200 adults from the high-diversity source population (Stephens Island) with $50 \%$ reproductive skew would retain $98.8 \% h$ and $83.2 \% A$ of the source (corresponding to a loss of $1.0 \% h$ and $11.2 \% A$ from the founder group), but a population founded with only 30 adults would retain $92.4 \% h$ and $52.4 \% A$ of the source population (a loss of $5.9 \% h$ and $18.5 \% A$ from the founders). Populations founded with 30 juveniles retained less $h$ and $A$ over 10 generations than those founded with 30 adults. 
Losses of allelic diversity from populations with low-diversity sources were small (6.5\%) under even the most extreme conditions (30 juvenile founders, $70 \%$ reproductive skew). On the other hand, populations with high-diversity sources lost at least $13.2 \%$ of alleles even under scenarios that promote retention of allelic diversity (200 adult founders, $0 \%$ reproductive skew, Table 5.2). Probabilities of extinction were $0 \%$ for all populations founded with 30,70 , or 200 adults. The probability of extinction was $0.4-4.8 \%$ for populations founded with 30 juveniles.

Table 5.2 Proportion of source heterozygosity $(h)$ and allelic diversity $(A)$ retained in founder groups of different sizes and composition ( $\mathrm{J}=$ juveniles, $\mathrm{Ad}=$ adults) and 10 generations after reintroduction. Three levels of reproductive skew $(70 \%, 50 \%$, and $0 \%)$ were modelled for each founder group. Allelic diversity was 14.4 in the high-diversity source population (representing Stephens Island) and 2.3 in the low-diversity source population (representing North Brother Island).

\begin{tabular}{|c|c|c|c|c|c|c|c|c|c|}
\hline & & \multicolumn{4}{|c|}{ High-diversity source } & \multicolumn{4}{|c|}{ Low-diversity source } \\
\hline & & \multirow{2}{*}{ Founders } & \multicolumn{3}{|c|}{10 generations } & \multirow{2}{*}{ Founders } & \multicolumn{3}{|c|}{10 generations } \\
\hline & & & 70 & 50 & 0 & & 70 & 50 & 0 \\
\hline \multirow{4}{*}{$h$} & $30 \mathrm{~J}$ & .983 & .860 & .906 & .937 & .983 & .856 & .900 & .934 \\
\hline & $30 \mathrm{Ad}$ & .983 & .883 & .924 & 949 & 983 & 875 & .918 & .945 \\
\hline & $70 \mathrm{Ad}$ & .993 & .952 & .967 & .978 & .993 & .944 & .961 & .974 \\
\hline & $200 \mathrm{Ad}$ & .998 & .982 & .988 & .992 & .998 & .972 & .980 & .986 \\
\hline \multirow{4}{*}{$A$} & $30 \mathrm{~J}$ & .709 & .431 & .485 & .548 & .992 & .935 & .955 & .972 \\
\hline & $30 \mathrm{Ad}$ & .709 & .466 & .524 & .593 & .992 & .948 & .962 & .981 \\
\hline & 70Ad & .843 & 639 & 686 & .747 & 1.00 & .984 & .990 & .997 \\
\hline & $200 \mathrm{Ad}$ & .944 & .798 & .832 & .868 & 1.00 & .997 & .999 & 1.00 \\
\hline
\end{tabular}

Greater male reproductive skew led to significant losses of $h$ and $A$ after 10 generations (Table 5.3). The loss of $h$ was between $0.2 \%$ and $2.9 \%$ greater with each $10 \%$ increase in reproductive skew (i.e. with 10\% more males excluded from mating), and was minimal after population size exceeded $\sim 250$ animals. The effect of reproductive skew on $h$ was slightly more severe when carrying capacity was smaller (Table 5.3). Reproductive skew had a greater effect on the loss of alleles in the high-diversity populations (Table 5.3). The loss of $A$ was $0.7-3.7 \%$ with each $10 \%$ increase in reproductive skew in the high-diversity populations, but $0.1-1.4 \%$ in the low-diversity populations. Populations founded with 30 adults from a high-diversity source population retained between $46.6 \%$ and $59.1 \%$ of the source $A$, depending on the degree of reproductive skew (Table 5.3).

The losses of $h$ and $A$ in density-dependent models were similar to, but slightly greater than losses predicted by the percentage of males mating at low density (Table 5.3). For 
example, with $20 \%$ reproductive skew at low density and $70 \%$ at high density, populations retained similar levels of $h$ and $A$ after 10 generations as predicted by density-independent models with $20-30 \%$ reproductive skew.

Table 5.3 Predicted proportion of source heterozygosity $(h)$ and allelic diversity $(A)$ retained 10 generations after reintroduction of 30 adult tuatara with different levels of male reproductive skew. The percentage of males excluded from mating (0-70\%) was varied under density-independent and density-dependent models. Allelic diversity was 14.4 in the high-diversity source population (representing Stephens Island) and 2.3 in the low-diversity source population (representing North Brother Island). Differences in values of $h$ between high- and low- diversity source result from differences in carrying capacities used in the models.

\begin{tabular}{lccccc}
\hline & \multicolumn{2}{c}{ High-diversity source } & & \multicolumn{2}{c}{ Low-diversity source } \\
\cline { 2 - 3 } \cline { 5 - 6 } & $h$ & $A$ & & $h$ & $A$ \\
\hline Density-independent & & & & & \\
70 & .883 & .466 & & .875 & .948 \\
60 & .911 & .503 & & .904 & .960 \\
50 & .924 & .524 & & .918 & .962 \\
40 & .932 & .543 & & .927 & .968 \\
30 & .938 & .561 & & .934 & .973 \\
20 & .943 & .574 & & .939 & .977 \\
10 & .947 & .584 & & .942 & .979 \\
0 & .949 & .593 & & .945 & .981 \\
& & & & & \\
Density-dependent & & & & & \\
$20^{1} / 70^{2}$ & .942 & .571 & & .934 & .972 \\
$0^{1} / 50^{2}$ & .949 & .593 & & .942 & .977 \\
\hline
\end{tabular}

1 proportion of males excluded from mating at low density

${ }^{2}$ proportion of males excluded from mating at high density

\subsection{Discussion}

Reintroduced populations of tuatara should retain a relatively high proportion of the heterozygosity (86-99\%) and allelic diversity (43-100\%) of their source populations after 10 generations. Founder groups released into Karori Wildlife Sanctuary, Titi Island, and Matiu/Somes Island have retained $84-100 \%$ allelic diversity of their source populations. Genetic diversity of the source populations affects the retention of diversity in the founders, with populations reintroduced from high-diversity sources (Stephens Island) losing more diversity than those from low-diversity sources (North Brother Island). As expected, larger founder groups increase the proportion of heterozygosity and allelic diversity of both the source populations and the founder groups retained after 10 generations. Greater male reproductive skew led to greater losses of heterozygosity and 
allelic diversity over 10 generations, but the effect of reproductive skew was minimal after the populations expanded to $>250$ animals.

\subsubsection{Losses of genetic diversity}

It is generally agreed that the more heterozygosity retained, the better (Franklin \& Frankham 1998; Lynch \& Lande 1998), but cited goals for genetic management of threatened species are typically $\sim 90-95 \%$ heterozygosity retained over 100-200 years (Soulé et al. 1986; Lacy 1987; Allendorf \& Ryman 2002). As tuatara are extremely longlived (possibly 100 years, Dawbin 1982; Cree 1994) with a long generation interval, targets for genetic management in reintroduced populations over 100 years have little meaning. However, we applied these targets (90-95\% heterozygosity) to tuatara over 10 generations.

The rate of population expansion has a large effect on how well genetic diversity is maintained, and factors that increase population growth rates will help to maintain genetic diversity (Chapter 4). After reintroduction, several factors may result in greater population growth rates. Release from competition results in higher body condition and increases in body size (Nelson et al. 2002a; McKenzie 2007). Female tuatara in better body condition may reproduce more frequently, and those with larger body size produce more eggs per clutch (Newman et al. 1994). Females in captivity, where resources are not limited, reproduce on average every two years (Moore et al. 2008). Additionally, at low density, the top predator of juveniles (adult tuatara) will be sparse, and juvenile survival may be higher. Under these conditions, the loss of heterozygosity and allelic diversity may be lower than predicted.

Genetic drift in small populations generally outweighs selection at functional loci (e.g. MHC, Miller \& Lambert 2004; Campos et al. 2006, but see Aguilar et al. 2004). In tuatara, the loss of genetic variation at MHC loci after a bottleneck is comparable to that lost at neutral loci (Miller et al. 2008). Although models of neutral genetic variation may have limited ability to predict the effects of a bottleneck on quantitative (polygenic) variation (Reed \& Frankham 2001), they can be used to evaluate how well adaptive variation (at least at $\mathrm{MHC}$ loci) may be maintained after reintroduction. The loss of genetic variation at these fitness-related genes may reduce the ability of the populations to respond to novel disease threats and increase the risk of an epidemic causing a population crash. 


\subsubsection{Choice of source population}

Populations reintroduced from North Brother Island would retain a larger proportion of the allelic diversity of the source than populations reintroduced from Stephens Island. However, this does not indicate that reintroduced populations from Stephens Island would have less adaptive potential. In fact, even when $50 \%$ of the original allelic diversity from Stephens Island is lost during reintroduction, it is higher than that on North Brother Island. Our results suggest that reintroductions from North Brother Island will lose little allelic diversity.

The assumption of no background inbreeding (i.e. all founders being unrelated) may be unrealistic, particularly for populations reintroduced from North Brother Island, which has a history of population bottlenecks and small population size (Newman 1877; Nelson et al. 2002b). We ignored inbreeding depression in our models, as it had relatively little impact on the amount of heterozygosity retained after 10 generations, but inbreeding (particularly when only a few founders are reintroduced) could affect individual fitness and may reduce population growth rates (Briskie \& Mackintosh 2004; Taylor et al. 2005). If deleterious alleles have been purged on North Brother Island because of its history of small population size and bottlenecks, then populations reintroduced from North Brother Island would be less affected by the increase in inbreeding than a population reintroduced from Stephens Island. However, purging is unlikely to reduce the negative effects of inbreeding (Ballou 1997; Frankham 2001), particularly when deleterious alleles have small rather than lethal effects (Hedrick 1994). Thus, it is likely that populations reintroduced from North Brother Island would be equally vulnerable to the potential effects of inbreeding. However, there are currently no known effects of inbreeding depression in this population (Mitchell et al. in review).

\subsubsection{Founder group size and composition}

Larger founder groups will help to maximise genetic diversity, reduce inbreeding, and maintain genetic diversity across generations. However, few founders are often released during reintroductions. For example, $46 \%$ of reintroductions of birds and mammals carried out between 1978 and 1986 had fewer than 30 founders (Griffith et al. 1989). Although the median number of founders increased subsequently to $\sim 50$ founders by 1993 (Wolf et al. 1996), founder groups are often still much smaller than 50, particularly 
for threatened species (e.g. Fitzsimmons et al. 1997; Towns \& Ferreira 2001). It has been suggested that $\sim 100$ founders would be needed to maximise the probability of success (Griffith et al. 1989), but species with high population growth rates may have a negligible probability of extinction through demographic stochasticity after reintroduction of as few as four animals (e.g. saddlebacks, Philesturnus caruncuatus, Taylor et al. 2005). Similarly, our models showed a negligible probability of extinction with at least 30 adult founders.

Captive-reared juveniles are often used as founders for reintroduced populations (Griffith et al. 1989), and it is often possible to release more captive-reared juveniles than wild founders without damaging the source populations. These juveniles are often related clutch-mates, which may accelerate inbreeding after reintroduction, but the harvest of an equivalent number of wild juveniles may be unfeasible for many cryptic species. Although head-started juveniles remain in captivity during the period of highest mortality (e.g. until age five for tuatara), survival of juveniles is lower than adults. Therefore, the release of juvenile founders would result in greater losses of genetic diversity than release of the same number of adults, because juvenile mortality effectively results in fewer founders. Additionally, reintroductions of juveniles have slightly higher probabilities of extinction (Nelson 1998; this study).

\subsubsection{Reproductive skew}

High variance in male reproductive success, where few males obtain almost all of the matings in a population, results in low effective population size (Nunney 1993; Parker \& Waite 1997). However, the effect of reproductive skew is difficult to quantify in species with overlapping generations. Our models showed that reproductive skew had relatively little impact on the loss of genetic diversity while population size was large. However, when population size is reduced (e.g. after reintroduction), reproductive skew has a significant impact on how well genetic diversity is maintained despite a long generation interval.

Differences in fitness due to unequal mating success is the basis of sexual selection, and is common in natural populations (Emlen \& Oring 1977). Thus, reproductive skew per se is not detrimental. However, following reintroduction, it may have a large influence on whether the genetic goals for management are met. Density-dependent changes in 
reproductive skew, where more males mate at low density, may facilitate the maintenance of genetic diversity in the early stages of population growth after reintroduction. Even if reproductive skew is high and density-independent, larger founder groups could be released to achieve genetic goals for management. Although it is possible to intentionally bias founder sex-ratios to account for reproductive skew (Lenz et al. 2007), it will not always be possible to determine which males might be successful, particularly if more males mate at low density or there are multiple determinants of male reproductive success (e.g. body size and colouration, Stapley \& Keogh 2006). For species with strong social structure where subordinate males may be entirely eliminated from mating, understanding how density influences social structure will be critical for understanding how genetic diversity is maintained and thus how reintroduction will impact population viability. 


\section{CHAPTER SIX}

\section{Managing genetic diversity in reintroduced populations: Thesis summary and applications}

\subsection{Introduction}

The loss of genetic diversity in a small reintroduced population could reduce the probability of establishment and persistence. Effective management of genetic diversity is thus important for the short- and long-term success of reintroduced populations. Using both empirical data and population modelling, I investigated factors that influence inbreeding dynamics and the retention of genetic diversity in translocated populations, and demonstrated the difficulty in applying that information for severely threatened species. Several features of species biology, including reproductive output, background inbreeding, and the mating system, may contribute to the maintenance or reduction of genetic diversity. Decisions on the design (e.g. the source population and composition of the founder group) and ongoing management (e.g. supplementation or serial translocation) of reintroduced populations also affect genetic diversity in the long-term. Patterns of genetic diversity among populations can be used to evaluate options for hybridising populations for reintroduction, which may provide a means of offsetting high background levels of inbreeding. Because understanding the effects that management actions have on genetic diversity is a priority for individual species recovery and the broader field of reintroduction biology (Towns 1999; Armstrong \& Seddon 2008), the results of this thesis addressed questions fundamental to conservation. Further, these results can be used to improve translocation planning and develop future research into how inbreeding may interact with demography and stochastic events to influence the risk of extinction after reintroduction.

\subsection{Summary of findings}

The main findings from each of the four data chapters (Chapters 2-6) are summarised below: 


\subsubsection{Assessing genetic diversity for conservation management: a case study of a threatened reptile (Chapter Two)}

Populations are often managed separately to avoid irreversibly mixing genetic lineages and to maintain the historic integrity of each population. Three remaining populations of Whitaker's skink (Cyclodina whitakeri), remnants of a once wider distribution, illustrate the conflict between this genetic goal with the more tangible and immediate threats of small population size and inbreeding. Populations of $C$. whitakeri from opposite ends of the species' range show marked differences at both mtDNA and microsatellite loci. However, this pattern is most likely an artefact of an historic genetic gradient coupled with rapid genetic drift. Animals in captivity show genetic signatures of both Pukerua Bay and island populations, despite the goal to maintain a pure Pukerua Bay stock for use as founders of a reintroduction. This mixed genetic stock provides an opportunity for the addition of skinks from Middle Island to the captive programme to evaluate the risks of further population hybridisation, while mitigating the risks of inbreeding.

\subsubsection{Genetic structure and individual fitness following translocation of a small lizard (Chapter Three)}

Translocation of pregnant females has been proposed as a means of maximising productivity and genetic diversity, but it is unclear whether the release of pregnant females reduces the genetic bottleneck and increases the effective population size. Fourteen years after reintroduction of 20 gravid females and 10 males, the population of Oligosoma suteri on Korapuki Island showed multiple genetic signatures of a bottleneck that were not detected in the source population on Green Island. However, the population on Korapuki Island has a significantly larger effective population size than would have been expected by translocation of 30 skinks. The translocation of gravid females aided in increasing the effective number of founders, indicating that it is a viable option for maximizing genetic diversity in translocated populations. No evidence of inbreeding depression is detectable using two performance-based surrogates of fitness. However, juvenile skinks on Korapuki Island are more homozygous than adults on Korapuki and Green Islands, indicating that juveniles with high homozygosity may not survive into adulthood. The continued loss of genetic variation and the loss of adaptive potential after reintroduction could not be assessed in the short-term. 


\subsubsection{The effects of supplementation and serial translocation on genetic diversity in species with varied life histories (Chapter Four)}

Maintaining genetic variation during the founding event is important for the success of translocated populations, but genetic drift and management actions may cause losses of genetic diversity after translocation. Three species of skinks with different life histories were translocated to Korapuki Island between 1988 and 1992. We sampled source and translocated populations of each species to assess the genetic consequences of translocation, and modelled genetic diversity over 10 generations to evaluate the implications of plausible management techniques. Translocation caused losses of heterozygosity and allelic diversity in all three species, but in the 14-18 years following translocation, the species with the lowest reproductive output (C. whitakeri) retained less heterozygosity than predicted by models. The species with the highest output (O. suteri) retained more than predicted. Supplementation of the translocated populations with 30 additional animals would not reduce the loss of heterozygosity over 10 generations in $O$. suteri, but would for species with lower reproductive output (C. whitakeri and C. alani) by decreasing the time required to reach carrying capacity. Harvest of $C$. whitakeri from Korapuki Island for a second-order translocation would accelerate the loss of heterozygosity after 10 generations in both the first- and second-order translocations. Therefore, supplementation of translocated populations will have the greatest effect during the period of population growth, as new animals will aid in rapid recovery from a bottleneck. Further, actions that slow population growth (e.g. harvesting from a relatively small population) should be avoided.

\subsubsection{How do reproductive skew and founder group size affect genetic diversity in reintroduced populations? (Chapter Five)}

Mating success is often unequal among individuals, which can lead to reduced effective population sizes. Using three reintroduced populations of tuatara (Sphenodon spp.), we evaluated how founder group size and variance in male reproductive success influence the long-term maintenance of genetic diversity after reintroduction. Reintroduced populations of tuatara should retain a relatively high proportion of the heterozygosity (85-99\%) and allelic diversity (43-100\%) of their source populations after 10 generations. The amount of genetic diversity in the source populations affects the retention of diversity in the founder group, with populations reintroduced from high diversity sources (e.g. Stephens Island) losing more diversity than those from low diversity sources (e.g. 
North Brother Island). Populations founded with more animals retain a greater proportion of the heterozygosity and allelic diversity of both their source populations and founder groups. Greater male reproductive skew leads to greater losses of genetic diversity over 10 generations, but reproductive skew only accelerates the loss of genetic diversity at small population size. Therefore, a reduction in reproductive skew at low density would facilitate the maintenance of genetic diversity in reintroduced populations. Even when reproductive skew is high and density-independent, larger founder groups could be released to achieve genetic goals for management.

\subsection{Implications for conservation management}

The results of this thesis can be applied toward conservation management of the specific populations studied and during the design of future reintroductions of any species. As reintroduction is one of the most commonly used tools in New Zealand conservation (Armstrong \& McLean 1995), and the number carried out annually is likely to increase (Saunders 1995), improving the maintenance of genetic diversity in reintroduced populations should be an important component of long-term management strategies. Effective management of genetic diversity must be considered in both the initial design and ongoing management of reintroduced populations.

\subsubsection{Minimising the initial genetic bottleneck}

Releasing gravid females would increase the effective population size after reintroduction (Chapter 3), despite concerns that stress during translocation may skew offspring sex ratios (e.g. Linklater 2007). Additionally, intentionally biasing the founder sex ratio towards females may help to preserve genetic diversity by increasing population growth rates (Lenz et al. 2007; Chapter 4). However, the population dynamics of the source must be carefully considered. Removal of a relatively large number of gravid females may not affect a large source population, but it could have detrimental demographic and genetic consequences on a small or recovering source population (e.g. the population of $C$. whitakeri on Korapuki Island or C. alani on Green Island, Chapter 4). Similarly, removal of a large number of females from an already male-biased population (e.g. tuatara on North Brother Island, Nelson et al. 2002b) could have devastating consequences for the source. These potential outcomes emphasize the need for extensive population surveys of the proposed sources prior to translocation. Particularly when reintroductions are carried out 
for the conservation management of threatened species, no source population should be harmed either demographically or genetically by the removal of animals for translocation (Marshall \& Spalton 2000).

Reproductive skew immediately after reintroduction reduces the amount of genetic diversity retained over 10 generations (Chapter Five). Knowing that variance in male reproductive success has long-term consequences will enable managers to be more selective about male founders during the translocation of highly polygynous species. During reintroduction of tuatara, for example, it may be advantageous to select males of similar body size in order to reduce high reproductive skew when the population is small and growing. However, density-dependent changes in the mating system, where more males mate at lower density (Kokko \& Rankin 2006; Moore et al. in review), may serve to equalise the genetic contributions of differently-sized males while populations are still small after reintroduction. Further, male tuatara grow rapidly in body size and condition after translocation (Nelson et al. 2002a), making the effects of translocating similarly-sized males difficult to predict. Research into how density influences social structure will be critical for understanding and predicting how reintroduction will impact population viability (see section 6.4.3).

\subsubsection{Maximising the retention of genetic diversity}

Although founder groups of 30 animals are adequate for reaching genetic goals for management of species with high reproductive output (e.g. O. suteri, Chapter 4), larger founder groups are required for species with lower reproductive output and/or low survival after release (e.g. C. whitakeri, Chapter 4). Further, because of low population growth rates, populations of $C$. whitakeri lose large amounts of genetic diversity in the 10 generations following release (Chapter 4).

Ongoing management of reintroduced populations should include monitoring and management for genetic diversity. For example, populations of C. whitakeri and C. alani on Korapuki Island show markedly lower genetic diversity than expected 14-18 years after release and have small effective population sizes, which resulted from low survivorship ( $C$. whitakeri) and a small release group (C. alani). Supplementation with additional animals from Middle Island to account for the small effective population sizes would increase genetic diversity, reduce inbreeding, and increase population growth rates (Chapter 4). 
Although negative consequences of inbreeding have not been noted in either population, it is clear that inbreeding has detrimental effects on individuals (e.g. Madsen et al. 1996; Coulson et al. 1999; Jamieson et al. 2007). Supplementation of C. alani on Korapuki Island would require hybridising populations, because the removal of skinks from Green Island (the source) most likely had negative demographic consequences for that small population. However, supplementation with C. alani from Middle Island would mimic the historic connectivity between the two island populations (Chapter 4). The proposed serial translocations of $C$. whitakeri and C. alani from Korapuki Island should not be carried out without first supplementing these populations and allowing them to recover. However, serial translocations of $O$. suteri may be feasible in the near future (Chapter 4).

\subsubsection{Management of highly inbred populations}

Many species and populations of strong conservation concern have high levels of background inbreeding (Madsen et al. 1996; Westemeier et al. 1998; Pimm et al. 2006). The small, declining population of $C$. whitakeri from Pukerua Bay is a clear case where the wild population has low genetic diversity and a history of inbreeding (Chapter 2 ). Inbreeding has been further accentuated in a captive population founded by just three wild animals. As 18 of the 21 animals in captivity are offspring of the captive breeding programme, most are full siblings (Chapter 2). Although a reintroduction from captivity to the wild poses the best option for management, up to 50 wild founders may be required to account for low rates of survival after release and low intrinsic rates of population growth (Towns \& Ferreira 2001; Chapter 4).

A translocation of $C$. whitakeri from Pukerua Bay has many challenging aspects. As many wild animals as possible should be represented in the founder group, but it would be best to release F1 offspring of the wild founders, rather than the wild animals themselves, so as to retain the wild animals in captivity and increase genetic variability in the breeding stock. The founder group for release should be comprised of as few full siblings as possible. As three additional animals have recently been caught at Pukerua Bay ${ }^{1}$, it may

\footnotetext{
${ }^{1}$ Three animals were caught by the Department of Conservation in December 2008, after publication of Chapter 2. They all share an mtDNA haplotype with all Pukerua Bay animals, and their microsatellite genotypes support the results presented in Chapter 2 (KA Miller, unpubl. data)
} 
become feasible to minimise the number of full-siblings in the founder group after $\sim 10$ years of captive breeding with a carefully designed breeding programme. The requisite number of wild founders could be obtained from Middle Island (Chapter 2), but the relative contribution of Middle Island animals to the founder group for reintroduction should be small (i.e. $<25 \%$ ), as very few animals are generally needed to restore genetic diversity and reduce the effects of inbreeding. For example, the release of only eight Texas panthers (Puma concolor stanleyana) into an inbred population of $<100$ Florida panthers (P. C. coryi) was sufficient to increase female reproductive output and kitten survival by up to $300 \%$ (Pimm et al. 2006). Further, intensive demographic and genetic monitoring after reintroduction should be high priorities for management.

\subsection{Future directions for research}

Although this thesis answers many questions regarding maximising genetic diversity in founder groups and the factors that may influence the maintenance of that diversity, it also presents several new questions. The research presented here provides a basis for further investigation on the ecological and genetic factors that influence population success after reintroduction. Further, it provides a foundation for the development of more complex models of losses of genetic diversity after translocation and how genetic drift may affect the long-term persistence of these valuable populations.

\subsubsection{Are the losses of genetic diversity consistent across reintroduced populations?}

A multitude of factors will influence the amount of genetic diversity retained in a reintroduced population in both the short- and long-term (Chapters 3-5), including the stochastic natures of population growth and inheritance. Therefore, it is important to understand how ecological factors and natural variation may influence the maintenance of genetic diversity. Several reintroductions of C. alani and C. whitakeri have been carried out within the Mercury Island group (both species were translocated to Korapuki, Stanley, and Red Mercury Islands). The closely monitored reintroduced populations on Korapuki Island clearly show losses of genetic diversity, and species with lower reproductive output show greater losses (Chapter 4). The other reintroduced populations were all founded with 30 animals, and provide an important comparison. Translocations of one species are often founded by different numbers of individuals. For example, reintroduced populations of North Island saddlebacks (Philesturnus carunculatus rufusater) were 
founded by 22-172 individuals (Lambert et al. 2005). By measuring the losses of genetic diversity in the reintroduced skink populations on Stanley and Red Mercury Islands founded with the same number of individuals, it may be possible to investigate how variation in survival and inheritance due to ecological and other factors affect the maintenance of diversity. Further, in species that are not severely threatened (e.g. O. suteri), translocations may be designed to experimentally test predictions about a loss of genetic diversity (Seddon et al. 2007). For example, reintroductions of $O$. suteri could be designed to experimentally test whether releasing gravid females always increases population growth rates and effective population size (Chapter 3 ).

\subsubsection{How is inbreeding depression manifested in reptiles?}

Inbreeding may have a variety of consequences for individuals and populations (Chapter 1), but can be difficult to detect in cryptic species, including many reptiles (Chapter 3 ). Developmental anomalies have occasionally been associated with inbreeding in reptiles (Schwaner 1990; Sarre \& Dearn 1991; Madsen et al. 1996; Gautschi et al. 2002), but more direct links between inbreeding and fitness have been noted less often (but see Madsen et al. 1996). Developmental stability, an individual's ability to withstand genetic and environmental perturbations, has been used as an indirect estimate of fitness (Leary et al. 1984). Anomalies are often associated with high homozygosity (Soulé 1979), and may be related to poor locomotor performance and survival of garter snakes (Thamnophis spp., Arnold \& Bennett 1988; Jayne \& Bennett 1990), but whether this relationship holds true across species is unclear. A better understanding of the links between developmental anomalies and biological fitness would reveal whether anomalies could be used to measure losses of fitness in populations. For cryptic species, using morphological measurements to infer inbreeding depression would be more feasible than measuring biological fitness. Oligosoma suteri would be an ideal candidate for such studies, as fluctuating asymmetry, or the deviation from bilateral symmetry, is unrelated to developmental stress and may be related to underlying genetic effects (Longson 2004). By combining genetic, morphological, and performance data, a link with fluctuating asymmetry may be uncovered. Further, an extensive genetic mark-recapture study of $O$. suteri on Korapuki Island (using genetic data from Chapter 3) would reveal whether either inbreeding and/or speed predict survival. 


\subsubsection{How do density-dependent factors operate after reintroduction?}

Many aspects of species biology are influenced by resource availability and population density (Kokko \& Rankin 2006), which in turn affect genetic diversity (Chapter 5). A more thorough understanding of the influence of these factors after reintroduction is critical in creating accurate models to predict population growth and extinction risk. For example, female tuatara may have greater reproductive output after reintroduction as a result of increased body condition or size (Nelson et al. 2002a), and more male tuatara may mate at lower density (Moore et al. in review). Further, hatching success and recruitment rates may be higher at lower density, as conspecific nest destruction and predation rates may be reduced. Although challenging, understanding how quickly and by how much reproductive output increases, how the mating system changes, and how recruitment rates change with density would provide much needed data for modelling population growth rates after reintroduction.

\subsubsection{How do demography and inbreeding interact to influence population persistence?}

The loss of genetic diversity combined with inbreeding depression in a small reintroduced population could reduce the probabilities of establishment and persistence (Armstrong \& Seddon 2008). Making predictions about this relationship requires estimating the effects of inbreeding depression and making projections about the severity of future threats. The probability of extinction may be negligible in a species with a high finite rate of population growth, even when egg failure rates are tripled because of inbreeding (e.g. saddlebacks, P. carunculatus, Taylor et al. 2005). However, extinction risk may be greatly influenced by inbreeding depression in species with lower reproductive output (Chapter 4) or high reproductive skew (Chapter 5), because of rapid increases in inbreeding. Using population modelling, links between inbreeding and the probability of population establishment could be clarified. Species with differing life histories (e.g. C. alani, C. whitakeri, and O. suteri, Chapter 4) and polygynous mating systems (e.g. tuatara, Chapter 5) provide the appropriate contrasts needed for such investigations. The insidious consequences of a loss of adaptive potential may have a large effect on the long-term persistence of these populations (Frankham 1999; Spielman et al. 2004). For example, incorporating novel disease threats in population models would allow a more thorough investigation of the long-term effect of the loss of genetic variation at functional loci (e.g. MHC genes). 


\subsection{Summary}

In summary, the commonly proposed founder group of 30 animals should result in reintroduced populations with high genetic diversity for species with high finite rates of population growth (Chapters 3-4). However, many reptile reintroductions in New Zealand fall short of this target (TP Bell, JM Germano, and KA Miller, unpubl. data). When fewer founders are released (e.g. C. alani on Korapuki Island), or when founder survival is low (e.g. C. whitakeri on Korapuki Island), genetic diversity in reintroduced populations is likely to fall well below the genetic targets for management (Chapter 4). Founder groups should therefore be larger for reintroductions of species with low reproductive output (Chapter 4), high mortality rates after release (Chapter 4), highly polygynous mating systems (Chapter 5), and high levels of background inbreeding (Chapters 2, 5). However, these species are also likely to be of greater conservation concern (Towns \& Ferreira 2001; Chapter 4), and fewer animals may be available for translocation. Ongoing genetic monitoring will be essential in the management of these populations. 


\section{References}

Aguilar A, G Roemer, S Debenham, M Binns, D Garcelon and RK Wayne (2004) High MHC diversity maintained by balancing selection in an otherwise genetically monomorphic mammal. Proc Natl Acad Sci USA 101: 3490-3494

Aitken N, JM Hay, SD Sarre, DM Lambert and CH Daugherty (2001) Microsatellite DNA markers for tuatara (Sphenodon spp.). Conserv Genet 2: 183-185

Allendorf FW (1986) Genetic drift and the loss of alleles versus heterozygosity. Zoo Biol 5: 181-190

Allendorf FW (2001) Genetics and the viability of insular populations of reptiles. N Z J Zool 21: 361

Allendorf FW and G Luikart (2007) Conservation and the Genetics of Populations. Blackwell, Oxford, UK

Allendorf FW and N Ryman (2002) The role of genetics in population viability. In: Population viability analysis (eds. SR Beissinger and DR McCullough), pp. 50-85. The University of Chicago Press, Chicago, USA

Amos W, JW Wilmer, K Fullard, TM Burg, JP Croxall, D Bloch and T Coulson (2001) The influence of parental relatedness on reproductive success. Proc R Soc Lond B 268: 2021-2027

Andrews RM, T Mathies and DA Warner (2000) Effect of incubation temperature on morphology, growth, and survival of juvenile Sceloporus undulatus. Herpetol Monogr: 420-431

Anon (1996) Conservation Management Strategy for Wellington 1996-2005. Department of Conservation, Wellington, New Zealand

Anthony LL and DT Blumstein (2000) Integrating behaviour into wildlife conservation: the multiple ways that behaviour can reduce $\mathrm{N}_{\mathrm{e}}$. Biol Conserv 95: 303-315

Aparicio JM, J Ortego and PJ Cordero (2006) What should we weigh to estimate heterozygosity, alleles or loci? Mol Ecol 15: 4659-4665

Armstrong DP and IG McLean (1995) New Zealand translocations: theory and practice. Pac Conserv Biol 2: 39-54

Armstrong DP and PJ Seddon (2008) Directions in reintroduction biology. Trends Ecol Evol 23: 2025

Arnold SJ and AF Bennett (1988) Behavioral variation in natural populations .v. morphological correlates of locomotion in the garter snake (Thamnophis radix). Biol J Linn Soc 34: 175-190

Ballou JD (1997) Ancestral inbreeding only minimally affects inbreeding depression in mammalian populations. J Hered 88: 169-178

Balloux F, W Amos and T Coulson (2004) Does heterozygosity estimate inbreeding in real populations? Mol Ecol 13: 3021-3031

Berry O, DM Gleeson and SD Sarre (2003) Microsatellite DNA markers for New Zealand skinks. Conserv Genet 4: 411-414 
Boessenkool S, SS Taylor, CK Tepolt, J Komdeur and IG Jamieson (2007) Large mainland populations of South Island robins retain greater genetic diversity than offshore island refuges. Conserv Genet 8: 705-714

Briskie JV and M Mackintosh (2004) Hatching failure increases with severity of population bottlenecks in birds. Proc Natl Acad Sci USA 101: 558-561

Brodie ED (1992) Correlational selection for color pattern and antipredator behavior in the garter snake Thamnophis ordinoides. Evolution 46: 1284-1298

Brown JL (1969) Territorial behavior and population regulation in birds. Wilson Bull 81: 293-329

Campos JL, D Posada and P Moran (2006) Genetic variation at MHC, mitochondrial and microsatellite loci in isolated populations of brown trout (Salmo trutta). Conserv Genet 7: 515530

Cassinello J, M Gomendio and ERS Roldan (2001) Relationship between coefficient of inbreeding and parasite burden in endangered gazelles. Conserv Biol 15: 1171-1174

Castanet J, DG Newman and H Saintgirons (1988) Skeletochronological data on the growth, age, and population structure of the tuatara, Sphenodon punctatus, on Stephens Island and Lady Alice Island, New Zealand. Herpetologica 44: 25-37

Censky EJ, K Hodge and J Dudley (1998) Over-water dispersal of lizards due to hurricanes. Nature 395: 556-556

Chapple DG, CH Daugherty and PA Ritchie (2008) Comparative phylogeography reveals predecline population structure of New Zealand Cyclodina (Reptilia: Scincidae) species. Biol J Linn Soc 95: 388-408

Chapuis MP and A Estoup (2007) Microsatellite null alleles and estimation of population differentiation. Mol Biol Evol 24: 621-631

Christian KA and CR Tracy (1981) The effect of the thermal environment on the ability of hatchling Galapagos land iguanas to avoid predation during dispersal. Oecologia 49: 218-223

Clement M, D Posada and KA Crandall (2000) TCS: a computer program to estimate gene genealogies. Mol Ecol 9: 1657-1659

Coltman DW, JG Pilkington, JA Smith and JM Pemberton (1999) Parasite-mediated selection against inbred Soay sheep in a free-living, island population. Evolution 53: 1259-1267

Coltman DW and J Slate (2003) Microsatellite measures of inbreeding: a meta-analysis. Evolution 57: $971-983$

Cornuet JM and G Luikart (1996) Description and power analysis of two tests for detecting recent population bottlenecks from allele frequency data. Genetics 144: 2001-2014

Coulson T, S Albon, J Slate and J Pemberton (1999) Microsatellite loci reveal sex-dependent responses to inbreeding and outbreeding in red deer calves. Evolution 53: 1951-1960

Craig J (1991) Are small populations viable? Acta XX Congressus Internationalis Ornithologici 4: 2546-2551

Crandall KA, ORP Bininda-Emonds, GM Mace and RK Wayne (2000) Considering evolutionary processes in conservation biology. Trends Ecol Evol 15: 290-295 
Cree A (1994) Low annual reproductive output in female reptiles from New Zealand. N Z J Zool 21: 351-372

Cree A and D Butler (1993) Tuatara Recovery Plan (Sphenodon spp.). Department of Conservation, Wellington, New Zealand

Cree A, JF Cockrem and LJ Guillette (1992) Reproductive cycles of male and female tuatara (Sphenodon punctatus) on Stephens Island, New Zealand. J Zool 226: 199-217

Cree A and CH Daugherty (1991) High rates of cutaneous water loss in nocturnal New Zealand reptiles. Unpublished report to the Director, Science and Research Directorate, Department of Conservation, Wellington, New Zealand

Crnokrak P and SCH Barrett (2002) Purging the genetic load: a review of the experimental evidence. Evolution 56: 2347-2358

Crnokrak P and DA Roff (1995) Dominance variance: associations with selection and fitness. Heredity 75: 530-540

Crnokrak P and DA Roff (1999) Inbreeding depression in the wild. Heredity 83: 260-270

Darwin C (1896) The Variation of Animals and Plants Under Domestication Vol. II. D Appleton \& Co., New York, USA

Daugherty CH, GB Patterson and RA Hitchmough (1994) Taxonomic and conservation review of the New Zealand herpetofauna. N Z J Zool 21:317-323

Daugherty CH, DR Towns, IAE Atkinson and GW Gibbs (1990) The significance of the biological resources of New Zealand islands for ecological restoration. In: Ecological Restoration of New Zealand Islands (eds. DR Towns, CH Daugherty and IAE Atkinson), pp. 9-21. Department of Conservation, Wellington, New Zealand

Dawbin WH (1982) The tuatara Sphenodon punctatus: aspects of life history, growth, and longevity. In: New Zealand herpetology (ed. DG Newman), pp. 237-250. New Zealand Wildlife Service, Wellington, New Zealand

de Guia APO and T Saitoh (2007) The gap between the concept and definitions in the evolutionarily significant unit: the need to integrate neutral genetic variation and adaptive variation. Ecol Res 22: 604-612

Di Rienzo A, AC Peterson, JC Garza, AM Valdes, M Slatkin and NB Freimer (1994) Mutational processes of simple sequence repeat loci in human populations. Proc Natl Acad Sci USA 91: 3166-3170

Dodd CK and RA Seigel (1991) Relocation, repatriation, and translocation of amphibians and reptiles: are they conservation strategies that work? Herpetologica 47: 336-350

Drayton JM, J Hunt, R Brooks and MD Jennions (2007) Sounds different: inbreeding depression in sexually selected traits in the cricket Teleogryllus commodus. J Evol Biol 20: 1138-1147

Earnhardt JM (1999) Reintroduction programmes: genetic trade-offs for populations. Anim Conserv 2: 279-286

Ebert D, C Haag, M Kirkpatrick, M Riek, JW Hottinger and VI Pajunen (2002) A selective advantage to immigrant genes in a Daphnia metapopulation. Science 295: 485-488

Edmands S (2007) Between a rock and a hard place: evaluating the relative risks of inbreeding and outbreeding for conservation and management. Mol Ecol 16: 463-475 
Emlen ST and LW Oring (1977) Ecology, sexual selection, and evolution of mating systems. Science 197: 215-223

Excoffier L, PE Smouse and JM Quattro (1992) Analysis of molecular variance inferred from metric distances among DNA haplotypes: application to human mitochondrial DNA restriction data. Genetics 131: 479-491

Fitzsimmons NN, SW Buskirk and MH Smith (1997) Genetic changes in reintroduced Rocky Mountain bighorn sheep populations. J Wildl Manage 61: 863-872

Frankham R (1999) Do population size bottlenecks reduce evolutionary potential? Anim Conserv 2: $255-260$

Frankham R (2001) Inbreeding and extinction: effects of purging. Conserv Genet 2: 279-285

Frankham R (2005) Genetics and extinction. Biol Conserv 126: 131-140

Franklin IR and R Frankham (1998) How large must populations be to retain evolutionary potential? Anim Conserv 1:69-73

Fraser DJ and L Bernatchez (2001) Adaptive evolutionary conservation: towards a unified concept for defining conservation units. Mol Ecol 10:2741-2752

Garland T, AF Bennett and CB Daniels (1990a) Heritability of locomotor performance and its correlates in a natural population. Experientia 46: 530-533

Garland T, E Hankins and RB Huey (1990b) Locomotor capacity and social dominance in male lizards. Funct Ecol 4: 243-250

Garza JC and EG Williamson (2001) Detection of reduction in population size using data from microsatellite loci. Mol Ecol 10: 305-318

Gautschi B, A Widmer, J Joshi and JC Koella (2002) Increased frequency of scale anomalies and loss of genetic variation in serially bottlenecked populations of the dice snake, Natrix tessellata. Conserv Genet 3: 235-245

Germano JM and PJ Bishop (2009) Suitability of amphibians and reptiles for translocation. Conserv Biol 23: 7-15

Gibbons JW, DE Scott, TJ Ryan, KA Buhlmann, TD Tuberville, BS Metts, JL Greene, T Mills, Y Leiden, S Poppy and CT Winne (2000) The global decline of reptiles, déjà vu amphibians. Bioscience 50: 653-666

Gilligan DM, DA Briscoe and R Frankham (2005) Comparative losses of quantitative and molecular genetic variation in finite populations of Drosophila melanogaster. Genet Res 85: 47-55

Gleeson DM, A Birkett, O Berry and D Chapple (2007) Population genetic structure of Oligosoma otagense (Otago skink) with comparisons to $O$. grande (grand skink) populations and the longterm genetic management of both species. N Z J Zool 34: 263

Goudet J (1995) FSTAT (Version 1.2): a computer program to calculate F-statistics. J Hered 86: 485486

Greaves SNJ, DG Chapple, DM Gleeson, CH Daugherty and PA Ritchie (2007) Phylogeography of the spotted skink (Oligosoma lineoocellatum) and green skink (O. chloronoton) species complex (Lacertilia: Scincidae) in New Zealand reveals pre-Pleistocene divergence. Mol Phylogen Evol 45: 729-739 
Greenwald OE (1974) Thermal dependence of striking and prey capture by gopher snakes. Copeia: $141-148$

Griffith B, JM Scott, JW Carpenter and C Reed (1989) Translocation as a species conservation tool: status and strategy. Science 245: 477-480

Grueber CE and IG Jamieson (2008) Quantifying and managing the loss of genetic variation in a free-ranging population of takahe through the use of pedigrees. Conserv Genet 9: 645-651

Guo SW and EA Thompson (1992) Performing the exact test of Hardy-Weinberg proportion for multiple alleles. Biometrics 48: 361-372

Hardy GS (1977) New Zealand Scincidae (Reptilia: Lacertilia): a taxonomic and zoogeographic study. N Z J Zool 4: 221-325

Hare KM, CH Daugherty and DG Chapple (2008) Comparative phylogeography of three skink species (Oligosoma moco, O. smithi, O. suteri; Reptilia: Scincidae) in northeastern New Zealand. Mol Phylogen Evol 46: 303-315

Hare KM and KA Miller (in review) What dives beneath: diving as a measure of performance in lizards. Herpetologica

Hay JM and DM Lambert (2008) Microsatellite DNA loci identify individuals and provide no evidence for multiple paternity in wild tuatara (Sphenodon: Reptilia). Conserv Genet 9: 10391043

Hayward BW (1986) Origin of the offshore islands of northern New Zealand and their landform development. New Zealand Department of Lands and Survey Information 16: 129-138

Hedrick P (2000) Genetics of Populations. Jones and Bartlett, Sudbury, USA

Hedrick PW (1994) Purging inbreeding depression and the probability of extinction: full-sib mating. Heredity 73: 363-372

Hedrick PW (1995) Gene flow and genetic restoration: the Florida panther as a case study. Conserv Biol 9: 996-1007

Hicks GRF, HP McColl, MJ Meads, GS Hardy and RJ Roser (1975) An ecological reconnaissance of Korapuki Island, Mercury Islands. Notornis 22: 195-220

Hill WG (1981) Estimation of effective population size from data on linkage disequilibrium. Genet Res 38: 209-216

Hitchmough RA, L Bull and P Cromarty (2007) New Zealand Threat Classification System lists. Science and Technical Publishing, Department of Conservation, Wellington, New Zealand

Hoare JM, LK Adams, LS Bull and DR Towns (2007) Attempting to manage complex predator-prey interactions fails to avert imminent extinction of a threatened New Zealand skink population. J Wildl Manage 71: 1576-1584

Hoare JM, S Pledger, SN Keall, J Nelson, NJ Mitchell and CH Daugherty (2006) Conservation implications of a long-term decline in body condition of the Brothers Island tuatara (Sphenodon guntheri). Anim Conserv 9: 456-462

Huey RB, W Schneider, GL Eire and RD Stevenson (1981) A field-portable racetrack and timer for measuring acceleration and speed of small cursorial animals. Experientia 37: 1356-1357 
Husak JF, SF Fox, MB Lovern and RA Van Den Bussche (2006) Faster lizards sire more offspring: sexual selection on whole-animal performance. Evolution 60: 2122-2130

Irschick DJ, JJ Meyers, JF Husak and JF Le Galliard (2008) How does selection operate on wholeorganism functional performance capacities? A review and synthesis. Evol Ecol Res 10: 177196

IUCN (1987) IUCN Position Statement on Translocation of Living Organisms: Introductions, reintroductions and re-stocking. (http://www.iucnsscrsg.org/)

Jamieson IG (2007) Has the debate over genetics and extinction of island endemics truly been resolved? Anim Conserv 10: 139-144

Jamieson IG, MS Roy and M Lettink (2003) Sex-specific consequences of recent inbreeding in an ancestrally inbred population of New Zealand takahe. Conserv Biol 17: 708-716

Jamieson IG, LN Tracy, D Fletcher and DP Armstrong (2007) Moderate inbreeding depression in a reintroduced population of North Island robins. Anim Conserv 10: 95-102

Jamieson IG, GP Wallis and JV Briskie (2006) Inbreeding and endangered species management: is New Zealand out of step with the rest of the world? Conserv Biol 20: 38-47

Jayne BC and AF Bennett (1990) Selection on locomotor performance capacity in a natural population of garter snakes. Evolution 44: 1204-1229

Jensen H, EM Bremset, TH Ringsby and BE Sæther (2007) Multilocus heterozygosity and inbreeding depression in an insular house sparrow metapopulation. Mol Ecol 16: 4066-4078

Keller LF (1998) Inbreeding and its fitness effects in an insular population of song sparrows (Melospiza melodia). Evolution 52: 240-250

Keller LF and DM Waller (2002) Inbreeding effects in wild populations. Trends Ecol Evol 17: 230-241

Keogh JS, IAW Scott and C Hayes (2005) Rapid and repeated origin of insular gigantism and dwarfism in Australian tiger snakes. Evolution 59: 226-233

Kokko H and DJ Rankin (2006) Lonely hearts or sex in the city? Density-dependent effects in mating systems. Philos Trans R Soc Lond B 361: 319-334

Kumar S, K Tamura and M Nei (2004) MEGA3: Integrated software for molecular evolutionary genetics analysis and sequence alignment. Brief Bioinform 5: 150-163

Kuo CH and FJ Janzen (2003) BOTTLESIM: a bottleneck simulation program for long-lived species with overlapping generations. Mol Ecol Notes 3: 669-673

La Marca E, KR Lips, S Lötters, R Puschendorf, R Ibáñez, JV Rueda-Almonacid, R Schulte, C Marty, F Castro, J Manzanilla-Puppo, JE García-Pérez, F Bolaños, G Chaves, JA Pounds, E Toral and BE Young (2005) Catastrophic population declines and extinctions in neotropical harlequin frogs (Bufonidae: Atelopus). Biotropica 37: 190-201

Lacy RC (1987) Loss of genetic diversity from managed populations: interacting effects of drift, mutation, immigration, selection, and population subdivision. Conserv Biol 1: 143-158

Lacy RC (1993) VORTEX - a computer simulation model for population viability analysis. Wildl Res 20: $45-65$ 
Lambert DM, T King, LD Shepherd, A Livingston, S Anderson and JL Craig (2005) Serial population bottlenecks and genetic variation: translocated populations of the New Zealand saddleback (Philesturnus carunculatus rufusater). Conserv Genet 6: 1-14

Leary RF, FW Allendorf and KL Knudsen (1984) Superior developmental stability of heterozygotes at enzyme loci in salmonid fishes. Am Nat 124: 540-551

Lenz TL, A Jacob and C Wedekind (2007) Manipulating sex ratio to increase population growth: the example of the Lesser Kestrel. Anim Conserv 10: 236-244

Linklater WL (2007) Translocation reverses birth sex ratio bias depending on its timing during gestation: evidence for the action of two sex-allocation mechanisms. Reprod Fertil Dev 19: 831-839

Lipsey MK and MF Child (2007) Combining the fields of reintroduction biology and restoration ecology. Conserv Biol 21: 1387-1388

Longson CG (2004) Fluctuating asymmetry is not a sensitive indicator of environmental stress in the egg-laying skink, Oligosoma suteri. N Z J Zool 31: 105

Lovegrove TG (1996) Island releases of saddlebacks Philesturnus carunculatus in New Zealand. Biol Conserv 77: 151-157

Luikart G, FW Allendorf, JM Cornuet and WB Sherwin (1998) Distortion of allele frequency distributions provides a test for recent population bottlenecks. J Hered 89: 238-247

Luikart G and JM Cornuet (1998) Empirical evaluation of a test for identifying recently bottlenecked populations from allele frequency data. Conserv Biol 12: 228-237

Luikart G, JM Cornuet and FW Allendorf (1999) Temporal changes in allele frequencies provide estimates of population bottleneck size. Conserv Biol 13:523-530

Lynch M and R Lande (1998) The critical effective size for a genetically secure population. Anim Conserv 1: 70-72

Macey JR, A Larson, NB Ananjeva, ZL Fang and TJ Papenfuss (1997) Two novel gene orders and the role of light-strand replication in rearrangement of the vertebrate mitochondrial genome. Mol Biol Evol 14: 91-104

Madsen T, R Shine, M Olsson and H Wittzell (1999) Restoration of an inbred adder population. Nature 402: 34-35

Madsen T, B Stille and R Shine (1996) Inbreeding depression in an isolated population of adders Vipera berus. Biol Conserv 75: 113-118

Maher CR and DF Lott (2000) A review of ecological determinants of territoriality within vertebrate species. Am Midl Nat 143: 1-29

Mansfield KG and ED Land (2002) Cryptorchidism in Florida panthers: prevalence, features, and influence of genetic restoration. J Wildl Dis 38: 693-698

Marr AB, LF Keller and P Arcese (2002) Heterosis and outbreeding depression in descendants of natural immigrants to an inbred population of song sparrows (Melospiza melodia). Evolution 56: 131-142

Marshall TC and JA Spalton (2000) Simultaneous inbreeding and outbreeding depression in reintroduced Arabian oryx. Anim Conserv 3: 241-248 
Maudet C, C Miller, B Bassano, C Breitenmoser-Würsten, D Gauthier, G Obexer-Ruff, J Michallet, P Taberlet and G Luikart (2002) Microsatellite DNA and recent statistical methods in wildlife conservation management: applications in Alpine ibex [Capra ibex (ibex)]. Mol Ecol 11: 421436

McKenzie KL (2007) Returning tuatara (Sphenodon punctatus) to the New Zealand mainland. MSc thesis. Victoria University of Wellington

McLain DK (1992) Population density and the intensity of sexual selection on body length in spatially or temporally restricted natural populations of a seed bug. Behav Ecol Sociobiol 30: 347-356

McNeely JA, KR Miller, WV Reid, RA Mittermeier and TB Werner (1990) Conserving the World's Biological Diversity. International Union for the Conservation of Nature and Natural Resources, World Resources Institute, Conservation International, and WWF - US and World Bank, Washington DC, USA

Meagher S, DJ Penn and WK Potts (2000) Male-male competition magnifies inbreeding depression in wild house mice. Proc Natl Acad Sci USA 97: 3324-3329

Merilä J and P Crnokrak (2001) Comparison of genetic differentiation at marker loci and quantitative traits. J Evol Biol 14: 892-903

Miller HC, M Andrews-Cookson and CH Daugherty (2007) Two patterns of variation among MHC class I loci in tuatara (Sphenodon punctatus). J Hered 98: 666-677

Miller HC and DM Lambert (2004) Genetic drift outweighs balancing selection in shaping postbottleneck major histocompatibility complex variation in New Zealand robins (Petroicidae). Mol Ecol 13: 3709-3721

Miller HC, KA Miller and CH Daugherty (2008) Reduced MHC variation in a threatened tuatara species. Anim Conserv 11:206-214

Miller HC, JA Moore, NJ Nelson and CH Daugherty (2009a) Influence of MHC genotype on mating success in a free-ranging reptile population. Proc R Soc Lond B 276: 1695-1704

Miller KA (2007) Taking the plunge. Forest and Bird 326: 20-22

Miller KA, DG Chapple, DR Towns, PA Ritchie and NJ Nelson (2009b) Assessing genetic diversity for conservation management: a case study of a threatened reptile. Anim Conserv 12: 163-171

Miller KA, KM Hare and NJ Nelson (in review-a) Alternate escape tactics may provide a means of compensation for poor performance ability. Biol J Linn Soc

Miller KA, NJ Nelson, PA Ritchie, FW Allendorf and DR Towns (in review-b) Genetic structure and individual fitness following translocation of a small lizard. Conserv Genet

Miskelly C (1999) Mana Island Restoration Plan. Department of Conservation, Wellington, New Zealand

Mitchell NJ, FW Allendorf, SN Keall, CH Daugherty and NJ Nelson (in review) Demographic effects of temperature-dependent sex determination: will tuatara survive global warming? Global Change Biol

Moore JA, CH Daugherty, SS Godfrey and NJ Nelson (in review) Social monogamy and multiple paternity in a wild population of a territorial reptile (tuatara). Biol J Linn Soc 
Moore JA, CH Daugherty and NJ Nelson (in press) Large male advantage: phenotypic and genetic correlates of territoriality in tuatara. J Herpetol

Moore JA, NJ Nelson, SN Keall and CH Daugherty (2008) Implications of social dominance and multiple paternity for the genetic diversity of a captive-bred reptile population (tuatara). Conserv Genet 9: 1243-1251

Moritz C (1994) Defining evolutionarily significant units for conservation. Trends Ecol Evol 9: 373375

Nelson NJ (1998) Conservation of the Brothers Island tuatara (Sphenodon guntheri). MConSc thesis. Victoria University of Wellington

Nelson NJ, SN Keall, D Brown and CH Daugherty (2002a) Establishing a new wild population of tuatara (Sphenodon guntheri). Conserv Biol 16: 887-894

Nelson NJ, SN Keall, S Pledger and CH Daugherty (2002b) Male-biased sex ratio in a small tuatara population. J Biogeogr 29: 633-640

Nelson NJ, MB Thompson, S Pledger, SN Keall and CH Daugherty (2004) Induction of oviposition produces smaller eggs in tuatara (Sphenodon punctatus). N Z J Zool 31: 283-289

Newman AK (1877) Notes on the physiology and anatomy of the tuatara (Sphenodon guntheri). Trans Proc N Z Inst 10: 222-239

Newman D and D Pilson (1997) Increased probability of extinction due to decreased genetic effective population size: experimental populations of Clarkia pulchella. Evolution 51: 354-362

Newman DG (1987) Burrow use and population densities of tuatara (Sphenodon punctatus) and how they are influenced by fairy prions (Pachyptila turtur) on Stephens Island, New Zealand. Herpetologica 43: 336-344

Newman DG, PR Watson and I McFadden (1994) Egg production by tuatara on Lady Alice and Stephens Island, New Zealand. N Z J Zool 21: 387-398

Nunney L (1993) The influence of mating system and overlapping generations on effective population size. Evolution 47: 1329-1341

O'Grady JJ, BW Brook, DH Reed, JD Ballou, DW Tonkyn and R Frankham (2006) Realistic levels of inbreeding depression strongly affect extinction risk in wild populations. Biol Conserv 133: 42-51

Paetkau D, R Slade, M Burden and A Estoup (2004) Genetic assignment methods for the direct, realtime estimation of migration rate: a simulation-based exploration of accuracy and power. Mol Ecol 13: 55-65

Paetkau D and C Strobeck (1995) The molecular basis and evolutionary history of a microsatellite null allele in bears. Mol Ecol 4: 519-520

Parker PG and TA Waite (1997) Mating systems, effective population size, and conservation of natural populations. In: Behavioral Approaches to Conservation in the Wild (eds. JR Clemmons and R Buchholz), pp. 243-261. Cambridge University Press, Cambridge, UK

Parkes J and E Murphy (2003) Management of introduced mammals in New Zealand. N Z J Zool 30: 335-359

Peakall R and PE Smouse (2006) GENALEX 6: genetic analysis in Excel. Population genetic software for teaching and research. Mol Ecol Notes 6: 288-295 
Pimm SL, L Dollar and OL Bass (2006) The genetic rescue of the Florida panther. Anim Conserv 9: 115-122

Piry S, A Alapetite, JM Cornuet, D Paetkau, L Baudouin and A Estoup (2004) GENECLASS2: a software for genetic assignment and first-generation migrant detection. J Hered 95: 536-539

Piry S, G Luikart and JM Cornuet (1999) BOTTLENECK: a computer program for detecting recent reductions in the effective population size using allele frequency data. J Hered 90: 502-503

Punzo F (2007) Sprint speed and degree of wariness in two populations of whiptail lizards (Aspidoscelis tesselata) (Squamata: Teiidae). Ethol Ecol Evol 19: 159-169

Ralls K and J Ballou (1986) Captive breeding programs for populations with a small number of founders. Trends Ecol Evol 1: 19-22

Ralls K, K Brugger and J Ballou (1979) Inbreeding and juvenile mortality in small populations of ungulates. Science 206: 1101-1103

Ramstad KM, CA Woody, GK Sage and FW Allendorf (2004) Founding events influence genetic population structure of sockeye salmon (Oncorhynchus nerka) in Lake Clark, Alaska. Mol Ecol 13: $277-290$

Reed DH and R Frankham (2001) How closely correlated are molecular and quantitative measures of genetic variation? A meta-analysis. Evolution 55: 1095-1103

Reed DH, EH Lowe, DA Briscoe and R Frankham (2003) Inbreeding and extinction: effects of rate of inbreeding. Conserv Genet 4: 405-410

Robb J (1986) New Zealand Amphibians and Reptiles in Colour. Collins, Auckland, New Zealand

Rousset $F$ (2008) GENEPOP'007: a complete re-implementation of the GENEPOP software for Windows and Linux. Mol Ecol Res 8: 103-106

Rozas J, JC Sanchez-DelBarrio, X Messeguer and R Rozas (2003) DnaSP, DNA polymorphism analyses by the coalescent and other methods. Bioinformatics 19: 2496-2497

Saccheri I, M Kuussaari, M Kankare, P Vikman, W Fortelius and I Hanski (1998) Inbreeding and extinction in a butterfly metapopulation. Nature 392: 491-494

Saccheri IJ, RA Nichols and PM Brakefield (2001) Effects of bottlenecks on quantitative genetic variation in the butterfly Bicyclus anynana. Genet Res 77: 167-181

Sadlier RA, SA Smith, AM Bauer and AH Whitaker (2004) A new genus and species of live-bearing scincid lizard (Reptilia: Scincidae) from New Caledonia. J Herpetol 38: 320-330

Sambrook J, EF Fritsch and T Maniatis (1989) Molecular Cloning: A Laboratory Manual. Cold Spring Harbor Laboratory Press, Cold Spring Harbor, USA

Sarre S and JM Dearn (1991) Morphological variation and fluctuating asymmetry among insular populations of the sleepy lizard, Trachydosaurus rugosus Gray (Squamata, Scincidae). Aust J Zool 39: 91-104

Saunders AJ (1995) Translocation in New Zealand: an overview. In: Reintroduction Biology of Australian and New Zealand Fauna (ed. M Serena), pp. 43-46. Surrey Beatty \& Sons, Chipping Norton, UK

Schrempf A, S Aron and J Heinze (2006) Sex determination and inbreeding depression in an ant with regular sib-mating. Heredity 97: 75-80 
Schuelke M (2000) An economic method for the fluorescent labeling of PCR fragments. Nat Biotechnol 18: 233-234

Schwaner TD (1990) Geographic variation in scale and skeletal anomalies of tiger snakes (Elapidae, Notechis scutatus-ater complex) in Southern Australia. Copeia: 1168-1173

Schwartz MW (1994) Conflicting goals for conserving biodiversity: issues of scale and value. Nat Areas J 14: 213-216

Seddon PJ, DP Armstrong and RF Maloney (2007) Developing the science of reintroduction biology. Conserv Biol 21: 303-312

Slate J, P David, KG Dodds, BA Veenvliet, BC Glass, TE Broad and JC McEwan (2004) Understanding the relationship between the inbreeding coefficient and multilocus heterozygosity: theoretical expectations and empirical data. Heredity 93: 255-265

Slate J, LEB Kruuk, TC Marshall, JM Pemberton and TH Clutton-Brock (2000) Inbreeding depression influences lifetime breeding success in a wild population of red deer (Cervus elaphus). Proc $\mathrm{R}$ Soc Lond B 267: 1657-1662

Slate J and JM Pemberton (2002) Comparing molecular measures for detecting inbreeding depression. J Evol Biol 15: 20-31

Snyder NFR, SR Derrickson, SR Beissinger, JW Wiley, TB Smith, WD Toone and B Miller (1996) Limitations of captive breeding in endangered species recovery. Conserv Biol 10: 338-348

Soulé M, M Gilpin, W Conway and T Foose (1986) The millennium ark: how long a voyage, how many staterooms, how many passengers? Zoo Biol 5: 101-113

Soulé ME (1979) Heterozygosity and developmental stability: another look. Evolution 33: 396-401

Southey IC (1985) The ecology of three rare skinks on Middle Island, Mercury Islands. MSc thesis. University of Auckland

Spielman D, BW Brook, DA Briscoe and R Frankham (2004) Does inbreeding and loss of genetic diversity decrease disease resistance? Conserv Genet 5: 439-448

Stapley J and JS Keogh (2006) Experimental and molecular evidence that body size and ventral colour interact to influence male reproductive success in a lizard. Ethol Ecol Evol 18: 275-288

Stephen CL, DG Whittaker, D Gillis, LL Cox and OE Rhodes (2005) Genetic consequences of reintroductions: an example from Oregon pronghorn antelope (Antilocapra americana). J Wildl Manage 69: 1463-1474

Stuart SN, JS Chanson, NA Cox, BE Young, ASL Rodrigues, DL Fischman and RW Waller (2004) Status and trends of amphibian declines and extinctions worldwide. Science 306: 1783-1786

Swanson BJ, LR Peters and CJ Kyle (2006) Demographic and genetic evaluation of an American marten reintroduction. J Mammal 87: 272-280

Swinnerton KJ, JJ Groombridge, CG Jones, RW Burn and Y Mungroo (2004) Inbreeding depression and founder diversity among captive and free-living populations of the endangered pink pigeon Columba mayeri. Anim Conserv 7: 353-364

Tallmon DA, A Koyuk, G Luikart and MA Beaumont (2008) ONeSAMP: a program to estimate effective population size using approximate Bayesian computation. Mol Ecol Res 8: 299-301 
Tallmon DA, G Luikart and RS Waples (2004) The alluring simplicity and complex reality of genetic rescue. Trends Ecol Evol 19: 489-496

Taylor BL and AE Dizon (1999) First policy then science: why a management unit based solely on genetic criteria cannot work. Mol Ecol 8: S11-S16

Taylor SS and IG Jamieson (2008) No evidence for loss of genetic variation following sequential translocations in extant populations of a genetically depauperate species. Mol Ecol 17: 545556

Taylor SS, IG Jamieson and DP Armstrong (2005) Successful island reintroductions of New Zealand robins and saddlebacks with small numbers of founders. Anim Conserv 8: 415-420

Thomas BW and AH Whitaker (1996) Translocation of the Fiordland skink Leiolopisma acrinasum to Hawea Island, Breaksea Sound, Fiordland, New Zealand. In: Reintroduction Biology of Australian and New Zealand Fauna (ed. M Serena), pp. 91-95. Surrey Beatty and Sons, Chipping Norton, UK

Thompson JD, TJ Gibson, F Plewniak, F Jeanmougin and DG Higgins (1997) The CLUSTAL_X windows interface: flexible strategies for multiple sequence alignment aided by quality analysis tools. Nucleic Acids Res 25: 4876-4882

Thorne ET and ES Williams (1988) Disease and endangered species: the black-footed ferret as a recent example. Conserv Biol 2: 66-74

Tocher MD and G Norbury (2005) Predicting extinction proneness and recovery in grand and Otago skinks. In: Kararehe Kino Vertebrate Pest Research (eds. J Coleman and C Thomson), pp. 1-3. RSNZ Publishing, Wellington, New Zealand

Towns DR (1975a) Ecology of the black shore skink, Leiolopisma suteri (Lacertilia: Scincidae), in boulder beach habitats. N Z J Zool 2: 389-407

Towns DR (1975b) Reproduction and growth of the black shore skink, Leiolopisma suteri (Lacertilia: Scincidae), in north-eastern New Zealand. N Z J Zool 2: 409-423

Towns DR (1991) Response of lizard assemblages in the Mercury Islands, New Zealand, to removal of an introduced rodent - the kiore (Rattus exulans). J R Soc N Z 21: 119-136

Towns DR (1992a) Distribution and abundance of lizards at Pukerua Bay, Wellington: implications for reserve management. Department of Conservation, Wellington, New Zealand

Towns DR (1992b) Recovery plan for Whitaker's skink and robust skink. Department of Conservation, Wellington, New Zealand

Towns DR (1994) The role of ecological restoration in the conservation of Whitaker's skink (Cyclodina whitakeri), a rare New Zealand lizard (Lacertilia: Scincidae). N Z J Zool 21: 457-471

Towns DR (1999) Cyclodina spp. Recovery Plan 1999-2004. Department of Conservation, Wellington, New Zealand

Towns DR (2002) Korapuki Island as a case study for restoration of insular ecosystems in New Zealand. J Biogeogr 29: 593-607

Towns DR and IAE Atkinson (2004) Restoration Plan for Korapuki Island (Mercury Islands), New Zealand, 2004-2024. Department of Conservation, Wellington, New Zealand 
Towns DR, IAE Atkinson and CH Daugherty (1990) The potential for ecological restoration in the Mercury Islands. In: Ecological Restoration of New Zealand Islands (eds. DR Towns, CH Daugherty and IAE Atkinson). Department of Conservation, Wellingtion, New Zealand

Towns DR and KG Broome (2003) From small Maria to massive Campbell: forty years of rat eradications from New Zealand islands. N Z J Zool 30: 377-398

Towns DR and CH Daugherty (1994) Patterns of range contractions and extinctions in the New Zealand herpetofauna following human colonization. N Z J Zool 21: 325-339

Towns DR and GP Elliott (1996) Effects of habitat structure on distribution and abundance of lizards at Pukerua Bay, Wellington, New Zealand. N Z J Ecol 20: 191-206

Towns DR and SM Ferreira (2001) Conservation of New Zealand lizards (Lacertilia: Scincidae) by translocation of small populations. Biol Conserv 98: 211-222

Towns DR, GR Parrish and I Westbrooke (2003) Inferring vulnerability to introduced predators without experimental demonstration: case study of Suter's skink in New Zealand. Conserv Biol 17: 1361-1371

Tsuji JS, RB Huey, FH Vanberkum, T Garland and RG Shaw (1989) Locomotor performance of hatchling fence lizards (Sceloporus occidentalis): quantitative genetics and morphometric correlates. Evol Ecol 3: 240-252

Van Damme R and B Vanhooydonck (2001) Origins of interspecific variation in lizard sprint capacity. Funct Ecol 15: 186-202

Van Oosterhout C and PM Brakefield (1999) Quantitative genetic variation in Bicyclus anynana metapopulations. Neth J Zool 49: 67-80

Van Oosterhout C, WF Hutchinson, DPM Wills and P Shipley (2004) MICRO-CHECKER: software for identifying and correcting genotyping errors in microsatellite data. Mol Ecol Notes 4: 535-538

Wang JL (2001) A pseudo-likelihood method for estimating effective population size from temporally spaced samples. Genet Res 78: 243-257

Wang JL, A Caballero, PD Keightley and WG Hill (1998) Bottleneck effect on genetic variance: a theoretical investigation of the role of dominance. Genetics 150: 435-447

Waples RS (1989) A generalized approach for estimating effective population size from temporal changes in allele frequency. Genetics 121: 379-391

Waples RS (1991) Pacific salmon, Oncorhynchus spp., and the definition of "species" under the Endangered Species Act. Mar Fish Rev 53: 11-22

Waples RS (2006) A bias correction for estimates of effective population size based on linkage disequilibrium at unlinked gene loci. Conserv Genet 7: 167-184

Waples RS and C Do (2008) LDNE: a program for estimating effective population size from data on linkage disequilibrium. Mol Ecol Res 8: 753-756

Weir BS and CC Cockerham (1984) Estimating F statistics for the analysis of population structure. Evolution 38: 1358-1370

Westemeier RL, JD Brawn, SA Simpson, TL Esker, RW Jansen, JW Walk, EL Kershner, JL Bouzat and KN Paige (1998) Tracking the long-term decline and recovery of an isolated population. Science 282: 1695-1698 
Whitaker AH (1968) Lizards of Poor Knights Islands, New Zealand. N Z J Sci 11: 623-651

Whitaker AH (1973) Lizard populations on islands with and without Polynesian rats, Rattus exulans (Peale). Proc N Z Ecol Soc 20: 121-130

Williams CL, TL Serfass, R Cogan and OE Rhodes (2002) Microsatellite variation in the reintroduced Pennsylvania elk herd. Mol Ecol 11: 1299-1310

Williams RN, OE Rhodes and TL Serfass (2000) Assessment of genetic variance among source and reintroduced fisher populations. J Mammal 81: 895-907

Wolf CM, B Griffith, C Reed and SA Temple (1996) Avian and mammalian translocations: update and reanalysis of 1987 survey data. Conserv Biol 10: 1142-1154

World Conservation Union (2008) IUCN Red List of Threatened Species. <www.iucnredlist.org> Accessed on 31 January 2008

Worthy TH (1987) Osteological observations on the larger species of the skink Cyclodina and the subfossil occurrence of these and the gecko Hoplodactylus duvaucelii in the North Island, New Zealand. N Z J Zool 14: 219-229 


\section{APPENDIX ONE}

\section{Alternate escape strategies may provide a means of compensation for poor performance ability}

\section{A1.1 Abstract}

Performance abilities can facilitate predator avoidance and consequently influence fitness, but determining the functional significance of any one antipredation tactic is difficult without understanding how alternate predator escape strategies relate. Maximal sprint speed and dive duration were measured in the semi-aquatic skink Oligosoma suteri to determine how morphology and behaviour influence these alternate predator escape mechanisms and the relationship between the two measures. Gravid females and juveniles ran significantly slower, but had equivalent or longer dive durations than males and non-gravid females. The two performance measures were not influenced by the same morphological and behavioural traits, and were not correlated among individuals, suggesting that the underlying traits that influence these performance capacities are unrelated. Although selection on sprint speed is likely mediated by predators, individuals that are poor sprinters because of their state (e.g. gravid individuals or those with tail loss) may have a greater likelihood of successful escape by adopting an alternate escape strategy. The use of alternate escape strategies may make it difficult to quantify selection on any one performance trait.

\section{A1.2 Introduction}

Whole-animal performance is influenced by a suite of underlying morphological, physiological, and behavioural traits (Arnold, 1983), and identifying which of these traits influence performance is essential in understanding phenotypic evolution. Selection may act directly on ecologically relevant abilities or on behavioural variations that influence performance (Arnold, 1983). Such organismal performance measures include swimming acceleration in fish (Ghalambor et al., 2004), flight speed in insects (Srygley \& Dudley, 1993) and birds (Veasey et al., 1998), and sprint speed in mammals (Iriarte-Diaz, 2002) and reptiles (Husak, 2006a). Selection on whole-animal performance is typically directional and has been quantified in several studies (see Irschick et al., 2008 for review). 
Performance ability can facilitate predator avoidance and consequently influence fitness (Lind \& Cresswell, 2005; Husak et al., 2006; Irschick et al., 2007; Irschick et al., 2008), yet animals may use several different strategies for the same function. For example, skylarks (Alauda arvensis) use song, refuge-seeking, and flocking to avoid predation, but the optimal escape option depends on body condition (Cresswell, 1994). Despite a species' potential use of multiple tactics, very few studies examine multiple, and very different, performance traits with the aim of understanding how selection on one may influence the other (but see Chapple \& Swain, 2002).

Determining the functional significance of the factors influencing any one antipredation tactic is difficult without understanding how alternate predator escape strategies relate (Lind \& Cresswell, 2005). If the same factors influence alternate tactics in a similar way, then individuals that perform well at one measure would perform well at both. Conversely, if the same factors influence alternate performance antagonistically, then individuals that perform well at one measure would perform poorly at the other. Finally, alternate measures may be influenced by entirely different underlying factors, or by different interactions of numerous factors, so that performance measures are not correlated, either phenotypically or genetically. As individuals often use multiple escape strategies under different conditions (e.g. Stapley \& Keogh, 2004), it may be difficult to resolve the influence of performance ability on fitness.

Physical or physiological factors may underlie trade-offs between performance and fecundity. For example, predation pressure favours increased swimming performance of Trinidadian guppies (Poecilia reticulate, in acceleration, speed, and travel distance) and simultaneously triggers an adaptive increase in reproductive allocation (\% body weight comprised of developing embryos). However, increased reproductive allocation concurrently reduces swimming performance, so that increased fecundity comes at a cost to escape performance (Ghalambor et al., 2004). Physical burdening of males by injection of a sterile fluid into the body cavity can reduce speed to an equivalent degree as gravidity in female garden skinks (Lampropholis guichenoti; Shine, 2003). Conversely, Olsson et al. (2000) found that the physiological burden of pregnancy (rather than physical burdening) reduces speed in pregnant snow skinks (Niveoscincus microlepidotus). Maternal speed is unrelated to litter mass, and non-pregnant females equivalent in mass to pregnant females run faster (Olsson et al., 2000). 
Sprint speed is perhaps the most commonly studied measure of locomotor performance in lizards (Van Damme \& Vanhooydonck, 2001); it is repeatable both across temperatures and between years (Huey \& Dunham, 1987), yet many morphological attributes, such as larger body size, tail length, and limb length, are positively correlated with speed. Speed influences biological fitness because of key roles in foraging, social interactions, and predator escape (Greenwald, 1974; Christian \& Tracy, 1981; Garland et al., 1990). However, as individuals in the field run faster when escaping a predator than when foraging (Husak, 2006b) and faster animals are more likely to survive attack than slower animals (Jayne \& Bennett, 1990; Warner \& Andrews, 2002; Husak, 2006a), speed when escaping is the more likely target of selection. Further, male collared lizards (Crotaphytus collaris) with faster maximum sprint speeds have larger territories and sire more offspring than slower males (Husak et al., 2006).

Many lizards also dive into water and submerge or swim as a means of predator escape (Pianka \& Vitt, 2003), yet the importance of aquatic escape mechanisms have rarely been studied (but see Lin et al., 2008; Hare \& Miller, in review). Voluntary (i.e. unrestrained) maximum dive time may be a relevant measure of performance for semi-aquatic species (Hare \& Miller, in review), but semi-aquatic lizards do not necessarily possess any unique physiological adaptations for diving (Daniels et al., 1987). Body size is positively correlated with oxygen stores and inversely correlated with metabolic rate; larger animals should therefore be able to dive for longer absolute periods. While this relationship holds true among endotherms (Schreer \& Kovacs, 1997), ectotherms challenge this assumption, as dive duration was not correlated with body size across 22 species of reptiles (Brischoux et al., 2008). However, the effect of body size on dive duration may be blurred in interspecific comparisons, and body size may have a strong intraspecific influence on diving capacity (Brischoux et al., 2008). Indeed, larger body size is related to an increased capacity for anaerobic metabolism in some ectotherms (McDonald et al., 1998).

We aimed to determine the relationship between alternate predator escape tactics that may be influenced by different underlying morphological, physiological, and behavioural traits. We used the semi-aquatic skink Oligosoma suteri (Boulenger) to examine relationships between maximal sprint speed and dive duration. To escape potential predators, $O$. suteri will run over short distances in search of cover and/or readily dive into intertidal marine waters (Whitaker, 1968; Towns, 1975a; Miller, 2007, Appendix 2). 
Specifically, we asked whether age and/or pregnancy status differentially affect these predator escape tactics and whether speed and dive time are positively correlated.

\section{A1.3 Methods}

\section{A1.3.1 Animal collection and husbandry}

We caught 149 O. suteri (117 adults and 32 juveniles) from Korapuki Island (18 ha, $36^{\circ} 39.5^{\prime} \mathrm{S}, 175^{\circ} 51^{\prime} \mathrm{E}$ ) in the Mercury Island Group, New Zealand. Oligosoma suteri are nocturnal, oviparous skinks that reach a maximum snout-vent length (SVL) of $108 \mathrm{~mm}$ (Hardy, 1977). They are restricted to boulder beaches and rocky platforms, residing close to the high water mark (Towns, 1975a). They are vulnerable to both terrestrial and avian predators, including tuatara (Sphenodon spp.), Australasian harriers (Circus approximans), gulls (Larus spp.), New Zealand kingfishers (Halcyon sancta), and native owls (Ninox novaeseelandiae; Whitaker, 1968). Lizards comprise a large portion of the diet of some of these predators (Hayes, 1991). Skinks were captured from 14 November to 5 December 2006 (late Austral spring) using pitfall traps baited with cat food and placed parallel to the high water mark.

Animals were housed individually in 2-L plastic containers with damp paper towels for 2-3 days (depending on their size) before performance trials to ensure all were in a postabsorptive state (Robert \& Thompson, 2000). All lizards defecated within this time period and did not defecate during or after the performance tests. Photoperiod was natural 14:10 light: dark (sunrise at $\sim 0600 \mathrm{~h}$ ) and ambient air temperature in the shade where experiments were conducted was $17.5 \pm 0.05 \mathrm{C}$. Temperatures during all trials were measured at $15 \mathrm{~min}$ intervals using thermal data loggers accurate to $0.3 \mathrm{C}$ (StowAway TidbiT, Onset Computer, Bourne MA). Data on the preferred body temperatures of freeranging $O$. suteri does not exist, but they reach maximum body temperatures of $25 \mathrm{C}$ in captivity and foraging ceases below 8-10 C (Towns, 1975a).

Standard morphometric measurements (mass, SVL, vent-tail length (VTL), tail regeneration length, and hind-limb length $(H L L)$ ) were taken prior to testing (Table A1.1). Hind-limb length is correlated with sprint speed in some lizards (Bonine \& Garland, 1999). Individuals were regarded as adults if over $75 \mathrm{~mm} \mathrm{SVL}$, which was the size of the smallest gravid female captured. Sex of adults was determined by eversion of the hemipenes in 


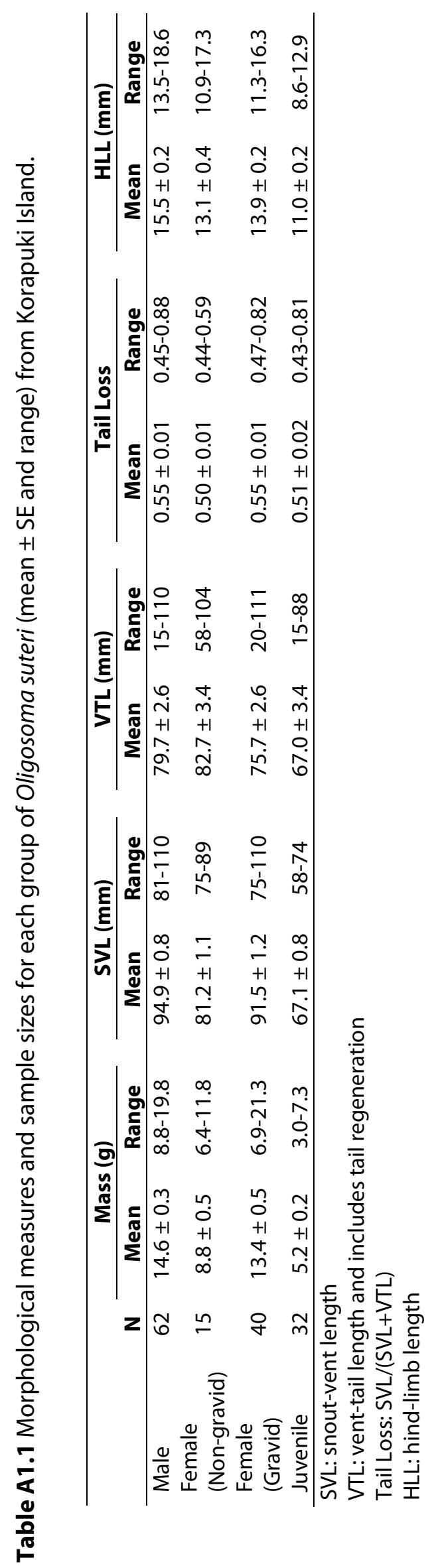


males; juvenile sex could not be determined. Gravidity status and an estimate of clutch size of females were determined by abdominal palpation. Ovulation of $O$. suteri occurs in late October and oviposition in late December (Towns, 1975b), so eggs were well-formed during the trapping period, and palpation is a reliable indicator of reproductive condition in Oligosoma species (Holmes \& Cree, 2006).

\section{A1.3.2 Sprint trials}

Sprint tests were conducted on a plastic racetrack $(80 \mathrm{~mm} \times 1.5 \mathrm{~m})$ with five paired infrared lights ( $0.25 \mathrm{~m}$ apart and $4 \mathrm{~mm}$ high) connected to a digital counter (modified from Huey et al., 1981) that were able to resolve the elapsed times between interruption of successive beams to $<1 \mathrm{~ms}$.

We conducted three trials per individual with at least 15 min rest between each trial. Animals were left undisturbed for $30 \mathrm{~min}$ before the first trial to acclimate to the ambient temperature. Sprinting was encouraged by touching the tail with a paintbrush. The maximum sprint speed over $0.25 \mathrm{~m}$ (instead of the available $1 \mathrm{~m}$ distance) was used, as this is a more ecologically relevant measure for $O$. suteri, which are typically found on rocky beaches where long sprints are not necessary to reach cover (Towns, 1975a). The fastest measure of speed across the three trials was used in the analyses. Speed was measured between $0840 \mathrm{~h}$ and $1620 \mathrm{~h}$ and the order of individuals was randomised over the three trials. Although 0 . suteri are recorded as nocturnal, $28 \%$ of adult captures in a previous study occurred during the day (K. Hare, unpublished data), suggesting that $O$. suteri are not strictly nocturnal. During sprint trials, some individuals paused while running, and we recorded the number of pauses over $1 \mathrm{~m}$. A pause was scored where an individual ran forwards after the tail was touched, then stopped and required another touch to continue running.

\section{A1.3.3 Dive trials}

Three voluntary dive trials were conducted per animal in an artificial rock pool between $1200 \mathrm{~h}$ and $2030 \mathrm{~h}$. All dive trials were conducted on the same day as, but after completion of, sprint trials; animals were given at least 120 min rest before dive trials were started, and were left undisturbed for 30 minutes before the first trial to acclimate to the ambient temperature. Animals were given at least $60 \mathrm{~min}$ rest between each dive trial, and the order of individuals was randomised over the three trials. 
The artificial rock pool was created using a darkened round plastic bin (base $340 \mathrm{~mm}$ diameter; $20 \mathrm{~L}$ to fill line) and rocks, seaweed, and intertidal seawater (temperature $16.7 \pm$ 0.07 C; modified from Hare \& Miller, in review). Rocks were placed so that one was above the water line as a diving platform, and animals were placed on this emergent rock facing away from the researcher. Diving was encouraged by tapping on the tail. Some skinks did not dive, and instead swam on the surface of the water. If an animal did not dive after 10 $s$, we removed it from the experiment. If an animal dived, we recorded whether it went on top of or beneath the substrate (seaweed or rocks), whether it moved while submerged (movement at least $50 \mathrm{~mm}$ ), and the time between submergence and voluntary emergence (i.e. the length of time that the animals had no access to air, hereafter "dive duration") using a manual stopwatch (accurate to $1 \mathrm{~s}$ ). Dive duration was a homologous trait across all individuals, as animals typically dived immediately after touching the tail and remained motionless for the entire dive, and never swam under water for more than a total of $5 \mathrm{~s}$ during the entire dive.

\section{A1.3.4 Statistical analysis}

All data were analysed using the statistical programmes $\mathrm{R}$ version 2.5.1 (R-DevelopmentCore-Team, 2007) and SPSS version 16.0, and significance was assumed at $P<0.05$. Data were tested for normality and are expressed as mean $\pm 1 \mathrm{SE}$. Data transformations were performed as required to meet assumptions of normality. Body condition was calculated as the residuals from fitted data using a linear regression of $\log$ (Mass) on $\log (\mathrm{SVL})$, and limb length relative to body size (hereafter 'limb length') was calculated as the residuals of $\log (\mathrm{HLL})$ on $\log (\mathrm{SVL})$.

We tested for the presence of correlations within an individual for sprint speed (and dive time) across the three sprint (and dive) trials using Pearson's correlation coefficients. $P$ values were obtained by using 1,000 permutation tests and adjusted using sequential Bonferroni adjustments. We also tested for potential correlations between an individual's maximum sprint speed and maximum dive duration using Pearson's correlation coefficient.

Animals were divided into four groups based on age, sex, and gravidity status: males, gravid females, non-gravid females, and juveniles (Table A1.1). We first tested whether 
groups differed in the tendency to pause during sprint trials, move during dive trials, or go beneath the substrate in dive trials (respectively) using a one-way ANOVA. Groups did not differ in these behavioural tendencies ( $P>0.05$ for all comparisons). We tested for differences in sprint speed and dive duration among all four groups with a one-way ANOVA, and post-hoc tests were conducted using Tukey's HSD. We then separately tested for differences among groups while accounting for differences in body size (see Table A1.1) using the residuals of sprint speed or dive duration on log(SVL).

As time of day influences swimming speed of elapid snakes (Llewelyn et al., 2006), we tested whether time of day influenced sprint speed or dive duration using a one-way ANOVA. The times of sprint trials were split into morning (0830-1230 h) and afternoon (1230-1630 h) periods, and the times of dive trials were split into afternoon (1200-1600 h) and early evening (1600-2030 h) periods. Time of day had no influence on performance in any group in either sprint or dive trials ( $P>0.05$ for all groups) and was not included in further analyses.

We analysed what factors may influence whether an animal dived or did not dive using generalized linear mixed effect models ( $\mathrm{glmm}$ ), which incorporate linear regressions while allowing for repeated measures from individuals. Data from all trials (three per individual) were used. Whether an animal dived or not was included as the binary response variable, and the covariates were SVL, body condition, a measure of the degree of tail loss (SVL/(SVL+VTL); hereafter referred to as tail loss), and maximum sprint speed. Individual was included as a random repeated variable, and age, sex, and gravidity status were included as a single factor (male, gravid female, non-gravid female, or juvenile).

We analysed morphological and behavioural factors influencing maximum sprint speed and dive duration separately for males, gravid females, and juveniles. We did not analyse the maximum performance of non-gravid females in relation to morphological and behavioural traits because of the small sample size $(n=15)$. We ran a series of standard least squares and reverse stepwise multiple regressions with the log of sprint speed and log of dive duration as dependent variables. The covariates were SVL, body condition, and tail loss. Clutch size was included as a factor for gravid females. We included limb length and whether an animal paused in models of sprint speed. In models of dive duration, we also included whether an animal moved or did not move while under water 
and whether an animal went beneath the substrate. If an animal did not dive in any trial, dive duration was treated as a missing value.

\section{A1.4 Results}

\section{A1.4.1 Sprint speed}

Maximum sprint speed ranged from $0.75-2.21 \mathrm{~ms}^{-1}$ for males, $0.70-1.33 \mathrm{~ms}^{-1}$ for gravid females, $0.90-2.37 \mathrm{~ms}^{-1}$ for non-gravid females, and 0.68-1.29 $\mathrm{ms}^{-1}$ for juveniles. Individual sprint speed was highly correlated across all trials $(r=0.43-0.62 ; P<0.001$ for all correlations). Thus, animals with high speeds in trial 1 had high speeds in trials 2 and 3.

Multiple regression did not produce a significant model for sprint speed in gravid females or juveniles ( $P=0.42$ and $P=0.40$, respectively; Table A1.2). A significant model was generated for male sprint speed $\left(F_{5,56}=3.31, P=0.01\right)$, and tail loss was the only predictor of speed (Table A1.2). Reverse stepwise regression resulted in a significant model that only retained tail loss and accounted for $18.2 \%$ of the total variation in male sprint speed. Males with shorter tails ran slower than males with longer tails $\left(F_{1,60}=14.58, P<0.001\right)$.

Table A1.2 Results of multiple regression models of maximum sprint speed and dive duration of Oligosoma suteri.

\begin{tabular}{|c|c|c|c|c|c|c|}
\hline \multirow{2}{*}{$\begin{array}{l}\text { Model } \\
\text { independent } \\
\text { variable }\end{array}$} & \multicolumn{2}{|c|}{$\begin{array}{c}\text { Males } \\
(\mathrm{N}=62)\end{array}$} & \multicolumn{2}{|c|}{$\begin{array}{c}\text { Gravid females } \\
(\mathrm{N}=40)\end{array}$} & \multicolumn{2}{|c|}{$\begin{array}{c}\text { Juveniles } \\
(\mathrm{N}=32)\end{array}$} \\
\hline & F ratio & $P$ value & F ratio & $P$ value & $F$ ratio & $P$ value \\
\hline Sprint Speed & 3.31 & 0.01 & 1.03 & 0.42 & 1.06 & 0.40 \\
\hline SVL & 2.40 & 0.13 & 1.34 & 0.26 & 0.008 & 0.93 \\
\hline Limb length & 0.97 & 0.33 & 0.04 & 0.84 & 2.17 & 0.15 \\
\hline Body Condition & 0.93 & 0.34 & 0.79 & 0.38 & 0.86 & 0.36 \\
\hline Tail Loss & 11.84 & 0.001 & 0.58 & 0.45 & 2.02 & 0.17 \\
\hline Clutch Size & na & na & 3.39 & 0.07 & na & na \\
\hline Pause $(\mathrm{Y} / \mathrm{N})$ & 0.38 & 0.54 & 0.04 & 0.84 & 0.25 & 0.62 \\
\hline Dive Duration & 3.25 & 0.01 & 0.32 & 0.92 & 1.82 & 0.15 \\
\hline SVL & 1.84 & 0.18 & 0.43 & 0.52 & 1.73 & 0.20 \\
\hline Body Condition & 0.15 & 0.70 & 0.02 & 0.90 & 0.05 & 0.83 \\
\hline Tail Loss & 0.78 & 0.38 & 0.01 & 0.92 & 0.30 & 0.59 \\
\hline Clutch Size & na & na & 0.06 & 0.81 & na & na \\
\hline Move $(\mathrm{Y} / \mathrm{N})$ & 0.35 & 0.56 & 0.62 & 0.44 & $<0.001$ & 0.99 \\
\hline Substrate $(\mathrm{Y} / \mathrm{N})$ & 13.1 & $<0.001$ & 0.80 & 0.38 & 7.01 & 0.01 \\
\hline
\end{tabular}




\section{A1.4.2 Dive duration}

Eighty-nine percent of skinks dived in at least one trial, 78\% of skinks dived in all three trials, and 16 skinks (11\%) did not dive in any trial. No morphological factors or maximum sprint speed explained whether an individual dived or not ( $P>0.10$ for all measures). Dive durations were highly correlated across all three trials $(r=0.63-0.69$, respectively; $P<$ 0.001 for all correlations). Maximum dive durations ranged from 20-893 s for males, 71 $1229 \mathrm{~s}$ for gravid females, 45-393 s for non-gravid females, and 19-811 s for juveniles.

Multiple regression did not produce a significant model for dive duration in gravid females ( $P=0.92 ;$ Table A1.2). A significant model was generated for male dive duration $\left(F_{5,47}=3.25, P=0.01\right)$; a nonsignificant model was generated for juvenile dive duration $\left(F_{5,25}=1.82, P=0.15\right)$, but whether an animal went beneath the substrate was significant (Table A1.2). Reverse stepwise regression resulted in significant models for male and juvenile dive duration that retained only whether an animal went beneath the substrate, and accounted for $22.7 \%$ and $18.0 \%$ of the variation in dive duration, respectively. Dive duration was significantly longer in males that went beneath the substrate $(334.24 \pm$ $38.49 \mathrm{~s})$ than males that stayed on top of the substrate $\left(91.56 \pm 21.50 \mathrm{~s} ; F_{1,51}=16.26, P<\right.$ $0.001)$. Similarly, dive duration was longer in juveniles that went beneath the substrate $(319.69 \pm 41.51 \mathrm{~s})$ than those that stayed on top of the substrate $\left(54.16 \pm 10.06 \mathrm{~s} ; F_{1,29}=\right.$ $7.58, P=0.01)$.

\section{A1.4.3 Relationship of Sprint Speed to Dive Duration}

Both sprint speed and dive duration differed significantly among males, gravid females, non-gravid females, and juveniles $(P<0.001$ and $P=0.03$, respectively; Figure $A 1.1)$. Gravid females had slower sprint speeds, but longer dive durations than males $(P<0.001$ and $P=0.04$, respectively) and non-gravid females $(P<0.001$ and $P=0.10$, respectively). The latter trend was not statistically significant, but the lack of significance was probably related to the small sample size of non-gravid females (dive durations were only obtained from 13 non-gravid females). Juveniles had slower sprint speeds than both males and non-gravid females ( $P<0.001$ and $P=0.003$, respectively), but similar sprint speeds to gravid females $(P=0.83)$. Mean dive duration of juveniles was not significantly different 


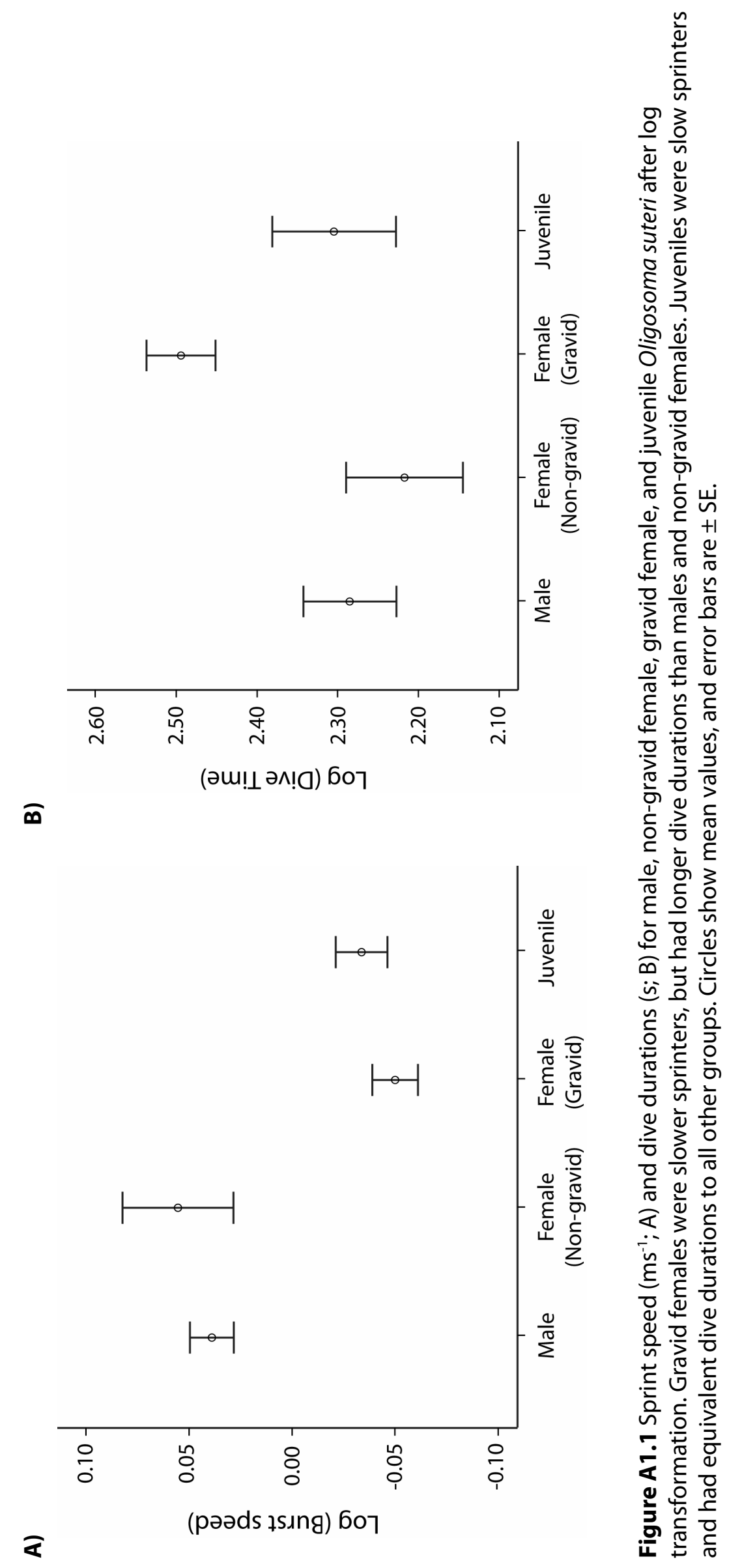


from gravid females $(P=0.17)$, males $(P=0.99)$ or non-gravid females $(P=0.89)$. Males and non-gravid females had similar sprint speeds and dive durations $(P=0.89$ and $P=0.94$, respectively). Significance did not change after correcting sprint speed and dive time for body size. The pattern observed for gravid females (slow runner, long diver) did not hold true at the individual level. Individual sprint speed and dive time were not correlated within any group or overall $(r=-0.30$ to $0.26, P>0.1$ for all correlations; Figure A1.2).

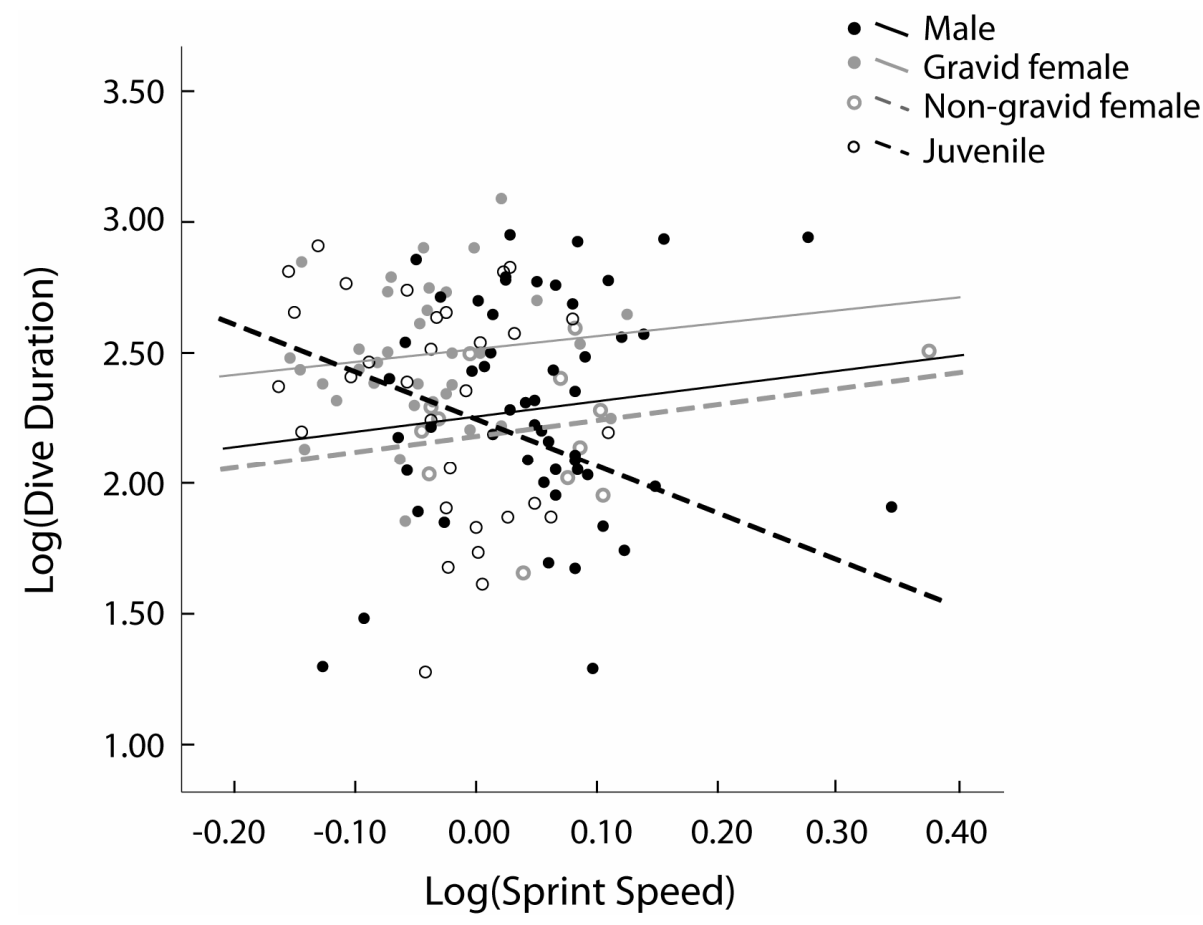

Figure A1.2 Relationship between maximum sprint speed and maximum dive duration of males, gravid females, non-gravid females, and juvenile Oligosoma suteri. Individual sprint speed and dive duration were not correlated within any group or overall $(r=-0.30$ to $0.26, P>0.1$ for all correlations).

\section{A1.5 Discussion}

Sprint speed and dive duration of a semi-aquatic lizard were not influenced by the same morphological and behavioural traits. Sprint speed and dive duration were not correlated among individuals, but animals of different age classes and gravidity status showed marked differences in mean sprint speed and dive duration. Gravid females and juveniles were poorer sprinters than males and non-gravid females, but both had equal or greater mean dive durations than males and non-gravid females. Thus, individuals that have slower sprint speeds because of gravidity or age may be able to compensate by relying more heavily on diving as a means of predator escape. 
Morphological differences among individuals can increase the variability of speed. Male O. suteri with shorter tails due to tail loss had lower speeds, probably caused by a biomechanical imbalance. While running, the tail is generally used to shift the animal's centre of gravity to its hind legs (Ballinger, Nietfeldt \& Krupa, 1979), and tail loss has commonly been associated with the reduction of sprint speed in lizards (Chapple \& Swain, 2002; Downes \& Shine, 2001; Punzo, 1982). Tail loss did not have a significant effect on sprint speed in gravid females, nor did any of the other measurements taken. Pregnancy frequently impairs locomotor performance and increases the risk of predation in many species (Olsson et al., 2000; Sinervo, Hedges \& Adolph, 1991), and pregnancy may have a greater influence on speed than other factors, including tail loss. Juvenile speed was not influenced by tail loss, but extensive tail loss was not common in juveniles (Table A1.1).

The wide range of dive durations seen in $O$. suteri may be due to the physiological capacity for apnoea not being taxed in some individuals. However, the high repeatability across all trials indicates that the physiological capacity of individuals was being reached. Still, we found no relationship between dive duration of males, gravid females, or juveniles, and any of the morphological measurements taken, despite predictions that intraspecific comparisons over a wide range of size and/or mass may reveal such links (Brischoux et al., 2008; Hare \& Miller, in review). However, individual performance is most likely to be best explained by direct physiological factors (e.g. the capacity for anaerobic metabolism) rather than by the predicted correlates of those factors (e.g. body size; McDonald et al., 1998). For example, weapon performance has a far stronger effect on the fitness of territorial lizards (C. collaris) than weapon size because of its direct influence on the outcomes of territory disputes (Lappin \& Husak, 2005).

Although there was no clear relationship between morphological measurements and dive duration, behavioural traits while diving had a strong and significant effect on dive duration in males and juveniles. By going underneath the substrate, animals may reduce their activity and exert less energy to remain underwater. Reduced activity would decrease metabolic demands and oxygen consumption (Wang et al., 1997; Williams et al., 1999), thereby allowing animals to dive for longer. Further, by going underneath the substrate, animals may be more cryptic, which would provide an additional measure of 
security against both terrestrial and aerial predators, particularly those that are able to catch prey in water (e.g. kingfishers).

Dive durations of juvenile and adult $O$. suteri in this study were equivalent (Figure A1.1b) and unrelated to body size. This contrasts with those of juvenile water skinks (Eulamprus quoyii), which dived for shorter periods of time than adults (Daniels, 1984). Species differences may result from sized-based differences (e.g. lung capacity) or from juvenile $E$. quoyii having limited experience with water; as juveniles, they are generally found further from water than adults (Daniels, 1984). All O. suteri likely have similar experience with rock pools. Juvenile O. suteri have frequent opportunities to dive, as they are relegated to areas of poor habitat close to the tide line (Towns, 1975a).

When close to the tide line, both running and diving are practicable strategies for escape. Importantly, diving performance in this study was unimpaired by the major morphological factor that impaired male speed (tail loss). Impairment of speed has been attributed to the increased risk of capture during an attack (Downes \& Shine, 2001), but in semi-aquatic species such as $O$. suteri, animals with poor speed because of tail loss may have a greater likelihood of successful escape via diving. Similarly, juveniles are generally poor sprinters (Figure A1.1a). Juvenile O. suteri may be slower than adults because of their small size and age (Hare et al., 2008), yet mean dive duration of juveniles was equivalent to males and non-gravid females. Thus, juveniles may have a greater likelihood of escape via diving relative to running.

Behavioural shifts may allow gravid females to avoid predation, despite the fact that gravidity impairs locomotor performance. Reproduction often comes at a cost to escape performance in many taxa (Ghalambor et al., 2004; Olsson et al., 2000), and as expected, gravid $O$. suteri had slower sprint speeds than non-gravid females and males. However, gravid females had longer dive times than both of these groups (Figure A1.1b). Reproduction may reduce escape performance via sprint speed, but physiological or physical changes during pregnancy may simultaneously improve alternate escape ability (i.e. dive duration). For example, an increase in mass or body condition associated with gravidity (physical change) may aid in remaining underwater.

Selection may act on performance differently under specific ecological or physiological conditions or may be constrained by the interaction of traits. Crypsis is a commonly 
employed strategy for predator avoidance in diverse taxa (Palma \& Steneck, 2001; Ruxton et al., 2004), and may serve as a third escape mechanism for O. suteri. Common lizards (Lacerta vivipara) rely heavily on crypsis during pregnancy to lessen their vulnerability to predation (Bauwens \& Thoen, 1981). Although we did not test for the use of crypsis in $O$. suteri, it is possible that individuals use crypsis during escape. If a group with impaired speed because of their state (e.g. juveniles, gravid females, or those with tail loss) relies on either alternate strategy (diving and/or crypsis) more than running, then selection on sprint speed would be less apparent in those groups.

Although there are clear patterns of performance abilities between age classes and animals of differing reproductive status, speed and dive time are not correlated among individual O. suteri (Figure A1.2). The two predator escape mechanisms may be unrelated morphologically and physiologically, as animals that perform well at one measure may or may not perform well at the other. Maximum sprint speed over short distances is influenced by the proportion of fast-twitch muscle fibres (Esbjornsson et al., 1993), but dive duration is likely related to maximum lung capacity and rate of oxygen consumption. Dive duration may also rely on endurance capacity. Sprint speed and endurance capacity, measured by running stamina, are negatively correlated among 12 species of lacertid lizards, though the mechanism underlying this trade-off is unclear (Vanhooydonck et al., 2001).

Importantly, sprint and dive performance were not correlated with each other, either positively or negatively. Thus, employment of an alternate escape strategy may therefore provide a means of compensation for poor performance ability when one type of performance is impaired. For semi-aquatic lizards, the factors that affect speed and dive duration will have a strong influence on an animal's vulnerability to predation. Investigating intraspecific variation in the physiological mechanisms underlying sprint speed and dive durations will allow a greater understanding of how these independent factors are integrated into whole-animal performance. Although selection on wholeanimal performance ability has been quantified in many studies, several studies have found no evidence for selection on performance traits (Irschick et al., 2008). Individuals with poor performance ability may have a greater likelihood of survival by adopting an alternate escape strategy. In cases where animals use multiple strategies (e.g. sprinting and diving) for the same function, detecting and quantifying selection on one trait (e.g. sprint speed) in isolation will be difficult. Understanding how selection acts on a suite of 
performance traits used for the same task may prove to be a worthy challenge. Field observations on the behavioural relationship between these escape mechanisms may clarify why individuals employ these alternative strategies in the face of predation. By investigating how predation risk is managed, we will more fully understand how the interaction of multiple predator escape tactics influences individual fitness.

\section{A1.6 Acknowledgements}

We are grateful to Dave Towns, Shay O'Neill, Chris Green, and lan Atkinson for assistance in the field, and the VUW herp hatchet group for comments on the manuscript. We thank the people of Ngāti Hei for their support of this research. This work was funded by grants from the New Zealand Department of Conservation, Victoria University of Wellington, and the Foundation for Research, Science and Technology (FRST). The work was approved by the Victoria University Animal Ethics Committee (2006R12) and the New Zealand Department of Conservation (permit: WK/19129/RES).

\section{References:}

Arnold SJ. 1983. Morphology, performance and fitness. American Zoologist 23: 347-361.

Ballinger RE, Nietfeldt JW, Krupa JJ. 1979. An experimental analysis of the role of the tail in attaining high running speed in Cnemidophorus sexlineatus (Reptilia: Squamata: Lacertilia). Herpetologica 35: 114-116.

Bauwens D, Thoen C. 1981. Escape tactics and vulnerability to predation associated with reproduction in the lizard Lacerta vivipara. Journal of Animal Ecology 50: 733743.

Bonine KE, Garland T. 1999. Sprint performance of phrynosomatid lizards, measured on a high-speed treadmill, correlates with hindlimb length. Journal of Zoology 248: 255-265.

Brischoux F, Bonnet X, Cook TR, Shine R. 2008. Allometry of diving capacities: ectothermy vs. endothermy. Journal of Evolutionary Biology 21: 324-329. 
Chapple DG, Swain R. 2002. Effect of caudal autonomy on locomotor performance in a viviparous skink, Niveoscincus metallicus. Functional Ecology 16: 817-825.

Christian KA, Tracy CR. 1981. The effect of the thermal environment on the ability of hatchling Galapagos land iguanas to avoid predation during dispersal. Oecologia 49: 218-223.

Cresswell W. 1994. Song as a pursuit-deterrent signal, and its occurrence relative to other anti-predation behaviors of skylark (Alauda arvensis) on attack by merlins (Falco columbarius). Behavioral Ecology and Sociobiology 34: 217-223.

Daniels CB. 1984. The adaptations to a riparian habitat by the water skink Sphenomorphus quoyii. Unpublished Ph.D. thesis, University of New England.

Daniels CB, Oakes N, Heatwole H. 1987. Physiological diving adaptations of the Australian water skink Sphenomorphus quoyii. Comparative Biochemistry and Physiology A - Physiology 88: 187-199.

Downes S, Shine R. 2001. Why does tail loss increase a lizard's later vulnerability to snake predators? Ecology 82: 1293-1303.

Esbjornsson M, Sylven C, Holm I, Jansson E. 1993. Fast-twitch fibers may predict anaerobic performance in both females and males. International Journal of Sports Medicine 14: 257-263.

Garland T, Hankins E, Huey RB. 1990. Locomotor capacity and social dominance in male lizards. Functional Ecology 4: 243-250.

Ghalambor CK, Reznick DN, Walker JA. 2004. Constraints on adaptive evolution: the functional trade-off between reproduction and fast-start swimming performance in the Trinidadian guppy (Poecilia reticulata). American Naturalist 164: 38-50.

Greenwald OE. 1974. Thermal dependence of striking and prey capture by gopher snakes. Copeia: 141-148. 
Hardy GS. 1977. New Zealand Scincidae (Reptilia: Lacertilia): a taxonomic and zoogeographic study. New Zealand Journal of Zoology 4: 221-325.

Hare KM, Miller KA. in review. What dives beneath: diving as a measure of performance in lizards. Herpetologica.

Hare KM, Pledger S, Daugherty CH. 2008. Low incubation temperatures negatively influence sprint speed and behaviour of the nocturnal lizard Oligosoma suteri (Lacertidae: Scincidae). Copeia 2008: 16-22.

Hayes LM. 1991. Behaviour of New Zealand kingfishers feeding chicks. Notornis 38: 7379.

Holmes KM, Cree A. 2006. Annual reproduction in females of a viviparous skink (Oligosoma maccanni) in a subalpine environment. Journal of Herpetology 40: $141-151$.

Huey RB, Dunham AE. 1987. Repeatability of locomotor performance in natural populations of the lizard Sceloporus merriami. Evolution 41: 1116-1120.

Huey RB, Schneider W, Eire GL, Stevenson RD. 1981. A field-portable racetrack and timer for measuring acceleration and speed of small cursorial animals. Experientia 37: 1356-1357.

Husak JF. 2006a. Does speed help you survive? A test with collared lizards of different ages. Functional Ecology 20: 174-179.

Husak JF. 2006b. Does survival depend on how fast you can run or how fast you do run? Functional Ecology 20: 1080-1086.

Husak JF, Fox SF, Lovern MB, Van Den Bussche RA. 2006. Faster lizards sire more offspring: sexual selection on whole-animal performance. Evolution 60: 21222130.

Iriarte-Diaz J. 2002. Differential scaling of locomotor performance in small and large terrestrial mammals. Journal of Experimental Biology 205: 2897-2908. 
Irschick D, Bailey JK, Schweitzer JA, Husak JF, Meyers JJ. 2007. New directions for studying selection in nature: studies of performance and communities. Physiological and Biochemical Zoology 80: 557-567.

Irschick DJ, Meyers JJ, Husak JF, Le Galliard JF. 2008. How does selection operate on whole-organism functional performance capacities? A review and synthesis. Evolutionary Ecology Research 10: 177-196.

Jayne BC, Bennett AF. 1990. Selection on locomotor performance capacity in a natural population of garter snakes. Evolution 44: 1204-1229.

Lappin AK, Husak JF. 2005. Weapon performance, not size, determines mating success and potential reproductive output in the collared lizard (Crotaphytus collaris). American Naturalist 166: 426-436.

Lin CX, Zhang L, Ji X. 2008. Influence of pregnancy on locomotor and feeding performances of the skink, Mabuya multifasciata: why do females shift thermal preferences when pregnant? Zoology 111: 188-195.

Lind J, Cresswell W. 2005. Determining the fitness consequences of antipredation behavior. Behavioral Ecology 16: 945-956.

Llewelyn J, Shine R, Webb JK. 2006. Time of testing affects locomotor performance in nocturnal versus diurnal snakes. Journal of Thermal Biology 31: 268-273.

McDonald DG, McFarlane WJ, Milligan CL. 1998. Anaerobic capacity and swim performance of juvenile salmonids. Canadian Journal of Fisheries and Aquatic Sciences 55: 1198-1207.

Miller KA. 2007. Taking the plunge. Forest and Bird 326: 20-22.

Olsson M, Shine R, Bak-Olsson E. 2000. Locomotor impairment of gravid lizards: is the burden physical or physiological? Journal of Evolutionary Biology 13: 263-268.

Palma AT, Steneck RS. 2001. Does variable coloration in juvenile marine crabs reduce risk of visual predation? Ecology 82: 2961-2967. 
Pianka ER, Vitt LJ. 2003. Lizards: Windows to the Evolution of Diversity. Univeristy of California Press: Los Angeles, USA.

Punzo F. 1982. Tail autotomy and running speed in the lizards Cophosaurus texanus and Uma notata. Journal of Herpetology 16: 329-331.

R-Development-Core-Team. 2007. R: A Language and Environment for Statistical Computing. 2.5.1 ed. Vienna, Austria: R Foundation for Statistical Computing.

Robert KA, Thompson MB. 2000. Influence of feeding on the metabolic rate of the lizard, Eulamprus tympanum. Copeia: 851-855.

Ruxton GD, Speed MP, Kelly DJ. 2004. What, if anything, is the adaptive function of countershading? Animal Behaviour 68: 445-451.

Schreer JF, Kovacs KM. 1997. Allometry of diving capacity in air-breathing vertebrates. Canadian Journal of Zoology/Revue Canadienne de Zoologie 75: 339-358.

Shine R. 2003. Effects of pregnancy on locomotor performance: an experimental study on lizards. Oecologia 136: 450-456.

Sinervo B, Hedges R, Adolph SC. 1991. Decreased sprint speed as a cost of reproduction in the lizard Sceloporus occidentalis: variation among populations. Journal of Experimental Biology 155: 323-336.

Srygley RB, Dudley R. 1993. Correlations of the position of center of body mass with butterfly escape tactics. Journal of Experimental Biology 174: 155-166.

Stapley J, Keogh JS. 2004. Exploratory and antipredator behaviours differ between territorial and nonterritorial male lizards. Animal Behaviour 68: 841-846.

Towns DR. 1975a. Ecology of the black shore skink, Leiolopisma suteri (Lacertilia: Scincidae), in boulder beach habitats. New Zealand Journal of Zoology 2: 389407. 
Towns DR. 1975b. Reproduction and growth of the black shore skink, Leiolopisma suteri (Lacertilia: Scincidae), in north-eastern New Zealand. New Zealand Journal of Zoology 2: 409-423.

Van Damme R, Vanhooydonck B. 2001. Origins of interspecific variation in lizard sprint capacity. Functional Ecology 15: 186-202.

Vanhooydonck B, Van Damme R, Aerts P. 2001. Speed and stamina trade-off in lacertid lizards. Evolution 55: 1040-1048.

Veasey JS, Metcalfe NB, Houston DC. 1998. A reassessment of the effect of body mass upon flight speed and predation risk in birds. Animal Behaviour 56: 883-889.

Wang T, Krosniunas EH, Hicks JW. 1997. The role of cardiac shunts in the regulation of arterial blood gases. American Zoologist 37: 12-22.

Warner DA, Andrews RM. 2002. Laboratory and field experiments identify sources of variation in phenotypes and survival of hatchling lizards. Biological Journal of the Linnean Society 76: 105-124.

Whitaker AH. 1968. Lizards of Poor Knights Islands, New Zealand. New Zealand Journal of Science 11: 623-651.

Williams TM, Haun JE, FriedI WA. 1999. The diving physiology of bottlenose dolphins (Tursiops truncatus) - I. Balancing the demands of exercise for energy conservation at depth. Journal of Experimental Biology 202: 2739-2748.

This appendix is in review as: Miller KA, KM Hare, and NJ Nelson (in review) Alternate escape strategies provide a means of compensation for poor performance ability. Biol J Linn Soc 
APPENDIX TWO

Taking the plunge: New Zealand's diving skinks

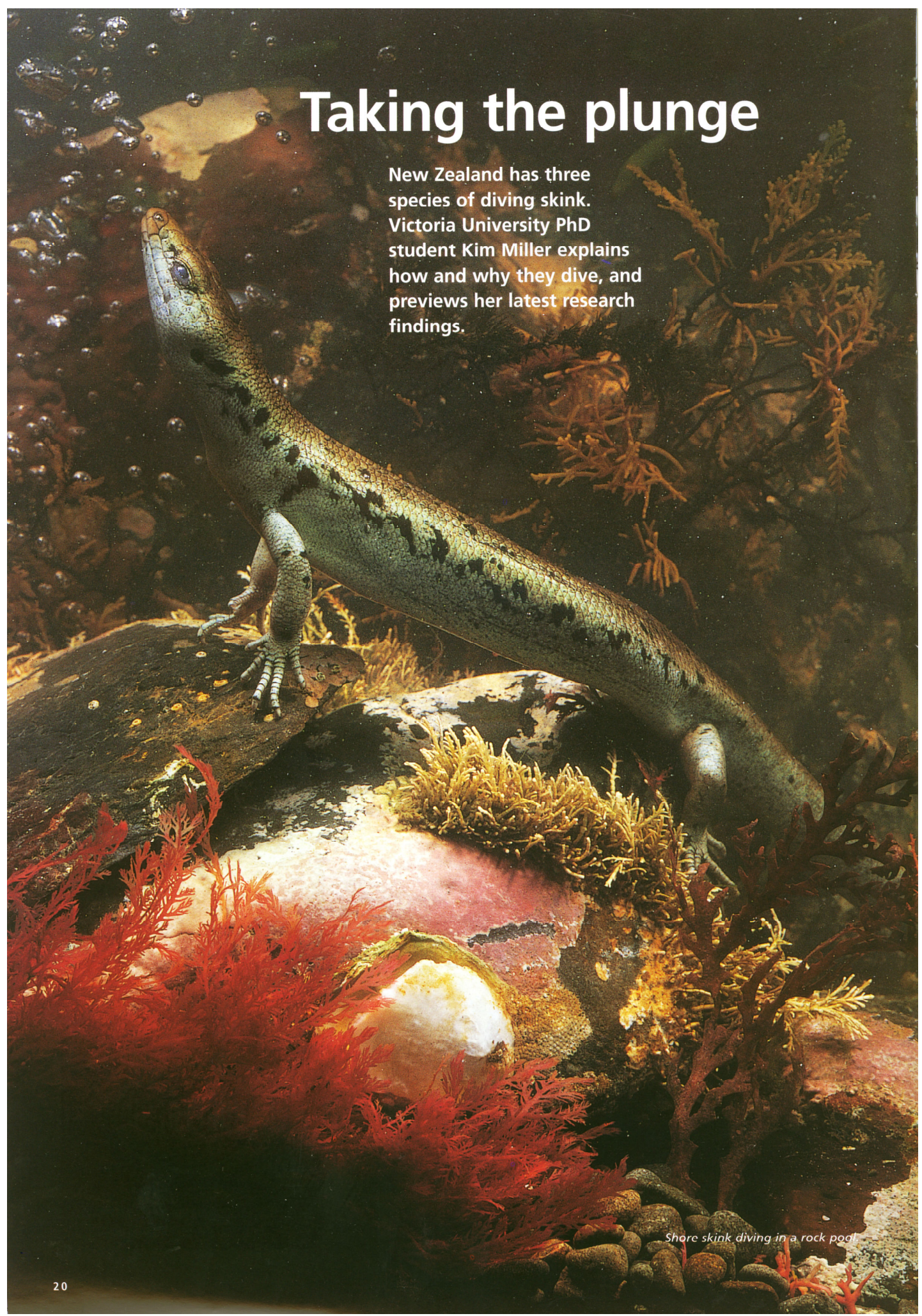


W E have all had the occasional glimpse of something small scurrying away at the beach or in the garden or on a forest walk, but we may not appreciate that something may be one of more than 80 species of native lizards.

New Zealand is a land of reptiles, yet a rustle in the grass or a dead tail-less offering from a cat is the only contact many people have with them.

Reptiles can be remarkably

inconspicuous and adept escape artists.

It's no surprise, then, that only a lucky few of us have seen or heard of the amazing diving abilities of a few of these elusive creatures.

Three species of New Zealand's shoredwelling skinks (mokomoko) are known to readily dive into seawater to forage for food or hide from predators. They are the Fiordland skink, which is restricted to south-west Fiordland and has been observed feeding underwater on marine invertebrates for up to five minutes, and the shore skink and egg-laying skink which live only on the coast of the mainland and offshore islands of the northern North Island. Both these latter species readily forage for invertebrates in rock pools on boulder beaches.

More impressive than diving for food are reports of the egg-laying skink plunging into rock pools to escape danger. This behaviour was first documented in 1968 by Tony Whitaker, a New Zealand reptile expert, who described it like this: "When disturbed these skinks dive into the nearest pool and either stay submerged or swim to the far side. While under water they may burrow under mud or stones or walk on the bottom, sometimes keeping their eyes open and turning their heads to follow movements above the surface."

He recorded "escape dives" of egglaying skinks at up to three minutes and 36 seconds. Shore skinks similarly plunge underwater when startled, but no dive lengths have been reported. In fact, skinks are generally well-adapted to diving and over 25 skink species worldwide dive regularly to evade predation. This behaviour is probably common in several more species.

Many terrestrial reptiles dive under water, including turtles, lizards, snakes, alligators, and crocodiles, but their activities can be very different. They primarily dive either to forage or escape predators.

These activities elicit very different physiological responses. During an escape dive reptiles undergo a slowing of the heart rate which is often accompanied by a cardiac shunt leading to pulmonary bypass. In contrast, dives for feeding and swimming generally don't result in a

prolonged cardiac shunt, but this depends on the level of activity.

www.forestandbird.org.nz

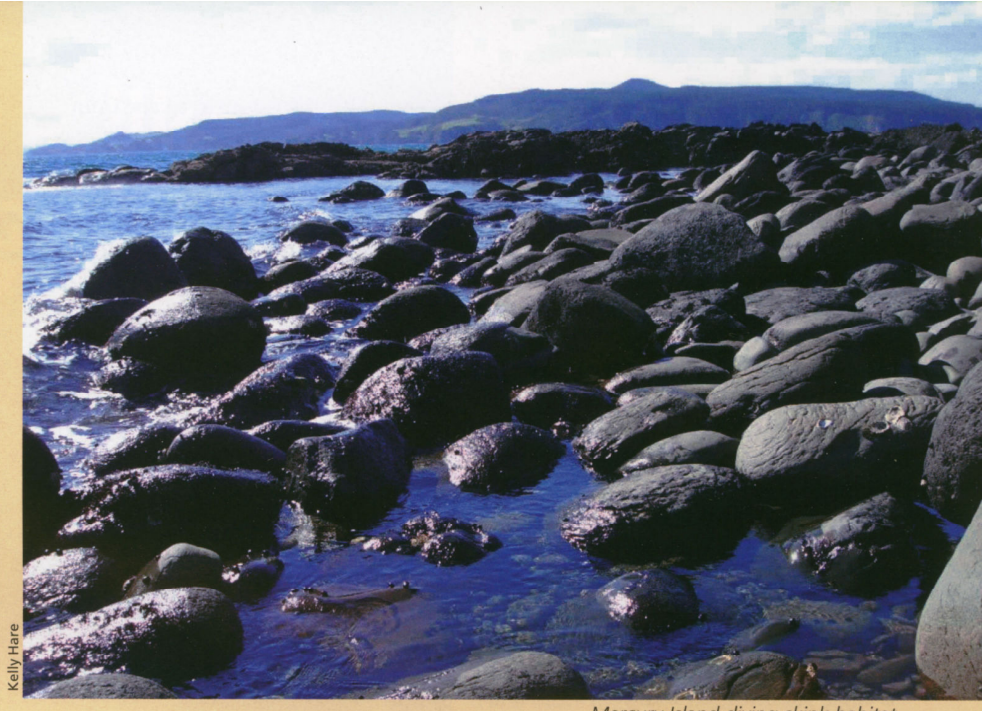

Although not unique to reptiles, these elaborate physiological responses are designed to slow the heart rate and decrease but the egg-laying skink is undoubtedly oxygen consumption while maximizing use a better diver. Shore skink dives average of the available oxygen. In essence, oxygen- about three minutes, and the longest dive poor blood from the right atrium, which would normally be sent to the lungs, is recycled back to arteries and capillaries and to the muscles. This right-to-left cardiac shunt allows leftover oxygen to be reused by the body instead of being exhaled.

Knowing that at least two of the three native skink species that dive for food also dive to escape predators, I set out with fellow Victoria University of Wellington researcher Kelly Hare to test the extent of the diving abilities of shore and egg-laying skinks.

On the Mercury Islands, east of the Coromandel Peninsula, we constructed an artificial rock pool using rocks, seaweed, and seawater. By mimicking the actions of a predator, we tested the diving ability of shore and egg-laying skinks.

After close to 750 trials, we began to see a clear picture of the capabilities of time was over six minutes. That record pales in comparison to egg-laying skinks, which average about six to seven minutes per dive. A female egg-laying skink won the record for maximum dive time by staying underwater for 20 minutes and 30 seconds!

Compare that to humans; most of us can only hold our breath for one or two minutes.

Even compared with other diving reptiles, the egg-laying skink fares well. Few reports of escape dive times exist, but most species have short escape dives. Green iguanas from Central and South America can escape predators by diving into water and remaining there for up to 30 minutes. But green iguanas are 10-15 times longer and more than 200 times heavier than egglaying and shore skinks.

In reptiles, both heart rate and respiratory rate are higher in smalle

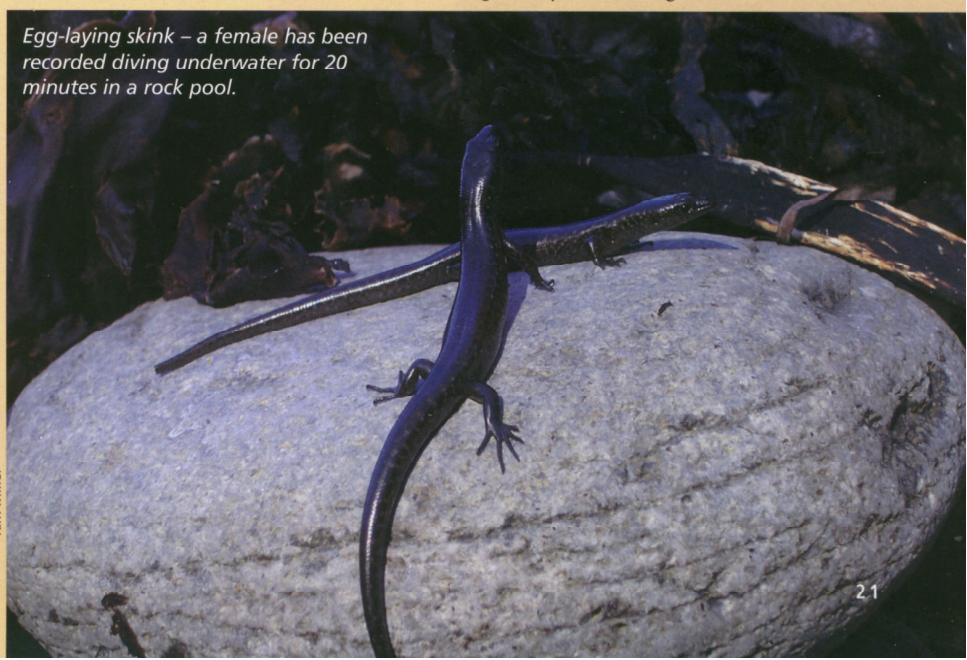


animals. Higher heart and respiratory rates usually reflect increased metabolic demands, so we might expect smaller lizards to have shorter dive times. It's not particularly surprising, then, that shore skinks (weighing about five grams) do not dive for as long as egg-laying skinks (about 12-20 grams), and that adults hold the records for both species.

But this is not a hard and fast rule. Some adult egg-laying skinks do not dive for as long as adult shore skinks, and some did not dive at all. About $10 \%$ of the animals lacked the motivation to dive and simply swam in the rock pool until they were scooped out.

Even with the occasional "swimmer" in their ranks, egg-laying skinks may be among the best divers in the skink world: The Australian eastern water skink is possibly the most studied diving skink. It is about the same size as the New Zealand egg-laying skink, but has maximum escape dives of about 13 minutes.

The story is quite different for foraging dives. While searching for food, the marine iguana of the Galápagos Islands regularly holds its breath for about 30 minutes. Freshwater crocodiles from the northern regions of Australia voluntarily hold their breath for up to two hours, and single dive of loggerhead turtles, occasional visitors to New Zealand waters, can last as long as seven hours!

Several other species of lizards are seen on beaches around New Zealand, including common geckos, brown skinks, common skinks and copper skinks. These species

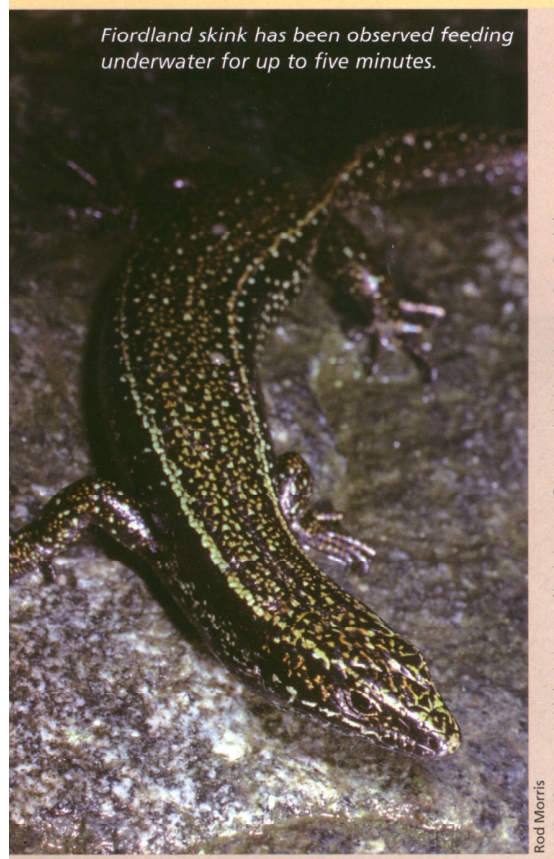

do not normally forage or escape from predators in the water and generally stay above the tide line. They live primarily in other habitats, but sometimes inhabit coastal vegetation and rocky beaches.

It is likely that a few more divers could be found among New Zealand's 80 or so lizard species. There has been only one documented report of juvenile chevron skinks diving in the wild, but there is good reason to suspect they may regularly use the water either for food or cover.

The chevron skink, a threatened species, is found only on Great and Little Barrier Islands and is most commonly found near stream margins, but small population sizes and severe range restriction have made them difficult to study. Unfortunately, it is not alone in that regard - over half of all New Zealand skink species are in need of urgent conservation action.

More than $40 \%$ of New Zealand lizard species, including the egg-laying skink, the Fiordland skink, and the chevron skink

are confined mainly or entirely to offshore islands.

Although mainland populations are few, at least 10 island populations of egg-laying skinks are expanding following the removal of introduced Pacific rats (kiore). Since 1992, a small population reintroduced by the Department of Conservation (DOC) to Korapuki Island off the Coromandel Peninsula has expanded to more than seven times its original size.

Fiordland skinks were reintroduced to Hawea Island, Breaksea Sound, in
1988 after the eradication of brown rats and the founding population of 40 adults expanded to over 200 animals within four years. These adept swimmers also re-colonised Breaksea Island from a neighbouring population after the eradication of brown rats.

Not all native reptiles are capable of self-reintroduction, and DOC is working to restore islands and translocate lizards to islands within their former range (see Forest \& Bird, February 1988). At least 15 different species of lizards have been reintroduced to islands around the country to date, and the future looks promising with many mainland sanctuaries now established.

Neither the shore skink nor the egglaying skink are currently classified as vulnerable, though they are difficult to see on the mainland. They are functioning parts of the coastal ecosystem on islands and have remarkable qualities. They eat carrion and invertebrates, and are a rich food source for several native bird species - if they don't plunge into a rock pool in time!

You may see lizards on the beach, but people should not try to make them dive. Not all lizards seen on the beach enjoy seawater. And all native reptiles in New Zealand are fully protected, so it is illegal to capture or harm them. If you sit back and wait, you might just be lucky enough to see one take the plunge.

Kim Miller is a PhD student in Conservation Biology at Victoria University of Wellington.

\section{Lizards on the shore}

B EACH-goers may see lizards on ither sandy or rocky beaches. Most of these lizards also live in other habitats and most are not adapted for diving. Here are some of the lizards you could encounter and where:

Common gecko (up to $160 \mathrm{~mm}$ ): North Island, widespread, especially in coastal regions; South Island, widespread; Stewart Island, rare. Nocturnal

Copper skink (up to $120 \mathrm{~mm}$ ): North Island only, widespread. Diurnal

${ }^{*}$ Fiordland skink (up to $170 \mathrm{~mm}$ ): South Island only, one mainland population in south-west Fiordland. Diurnal.

Common skink (up to $160 \mathrm{~mm}$ ): North Island, Hawkes Bay and central to southern parts; South Island, widespread; Stewart Island. Diurnal

${ }^{*}$ Shore skink ( up to $150 \mathrm{~mm}$ ): North Island only, east coast north of Gisborne, west coast mainly north of Auckland. Diurna
${ }^{*}$ Egg-laying skink (up to $225 \mathrm{~mm}$ ): North Island only, 3 mainland populations, Coromandel to north of Auckland. Nocturnal

Brown skink (up to $150 \mathrm{~mm}$ ): North Island, west from Taranaki to Wellington; South Island, Marlborough Sounds, Nelson, north Westland. Diurnal.

*Diving skink species

Male shore skink at night in rock pool.

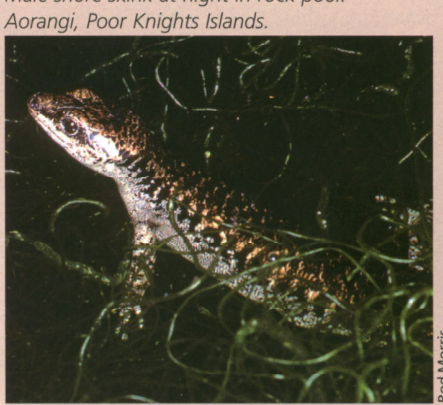

